\title{
A COMPUTATIONAL STUDY OF ENGINE DEFLECTION USING A CIRCULATION CONTROL WING
}

\author{
A Thesis \\ presented to \\ the Faculty of California Polytechnic State University, \\ San Luis Obispo
}

\author{
In Partial Fulfillment \\ of the Requirements for the Degree \\ Master of Science in Aerospace Engineering
}

by

Bryan H. Blessing

May 2011 
(C) 2011

Bryan H. Blessing

ALL RIGHTS RESERVED 


\section{COMMITTEE MEMBERSHIP}

TITLE :

A COMPUTATIONAL STUDY OF ENGINE DEFLECTION USING A CIRCULATION CONTROL WING

\section{AUTHOR: Bryan H. Blessing}

DATE SUBMITED: May 2011

Dr. David D. Marshall

Advisor and Committee Chair

Aerospace Engineering Dept.

Dr. Rob A. McDonald

Committee Member

Aerospace Engineering Dept.

Dr. Kim A. Shollenberger

Committee Member

Mechanical Engineering Dept.

Dr. Jin Tso

Committee Member

Aerospace Engineering Dept. 


\section{ABSTRACT \\ A COMPUTATIONAL STUDY OF ENGINE DEFLECTION USING A CIRCULATION CONTROL WING}

Bryan H. Blessing

In the past, research into Short Takeoff and Landing aircraft has led to the investigation of the coupling of a Circulation Control Wing and Upper Surface Blowing engine. The Circulation Control Wing entrains the flow of the engine to be deflected downward such that a component of the thrust is now in the vertical direction. The unfortunate consequence of the Upper Surface Blowing engine is the poor cruise performance due to scrub drag. Cal Poly's research into a Cruise Efficient Short Takeoff and Landing Aircraft offers a solution by pylon mounting over the wing engines. Analysis shows that the engine thrust is still deflected downward resulting in very high lift coefficients above 6.6. In the culmination of this project Cal Poly would like to find a correlation between the location of the engine and the deflection angle of the thrust.

The results of this study show some engine deflection for an over the wing engine. The configurations explored were able to provide $3-8.5^{\circ}$ of deflection. The deflection falls short of the results by previous static and wind tunnel tests of upper surface blowing engines. The results show that the closer to the wing and further forward the engine is located the more engine deflection will be seen. This paper explores the trends of coupling an over the wing engine with a circulation control wing as well as compare the results to the idealized claims of previous experiments. 


\section{ACKNOWLEDGMENTS}

First and foremost I want to thank my advisor Dr. David Marshall for his assistance in funding and encouraging my work on this project. Without his guidance and funding this work would not have been possible. This work was funded as part of a NASA Research Announcement award under Contract \# NNL07AA55C with Craig Hange and Joe Posey as the technical monitors.

I wish to thank the excellent work of David Hall from DHC Engineering, Inc. in developing the design of the aircraft that was the basis of this work. A special thanks to Rory Golden and Nick Paciano for their help in generating the geometry for this work. To all others in the aerospace graduate lab whose late night coffee runs and encouragement kept me working late into the nights.

To my many friends and family who put up with my neurotic disposition in this long drawn out process of finishing this work, I want to thank you for all your support. I owe many of you a beer. I made many life-long friends in college and I look forward to the many more stories and adventures we will have in the future.

Thank you to my parents who have always stood behind me with their full support while I meandered my way through school and life. I am grateful for your support

over the last 8 years. This thesis is a small testament to your parenting and guidance through my adolescence and because of your parenting I am better prepared to hit life head on. 


\section{Table of Contents}

List of Tables viii

List of Figures $\quad$ X

Nomenclature $\quad$ xi

1 Introduction $\quad \mathbf{1}$

1.1 Why is There a Need for Engine Deflection? . . . . . . . . . . . . 1

1.2 Background . . . . . . . . . . . . . . . . . . . 2

1.2.1 Upper Surface Blowing Technology . . . . . . . . . . . . . 2

1.2.2 Circulation Control Technology ...................... 4

1.2.3 Engine Deflection . . . . . . . . . . . . . . 6

1.2.4 Brief History of Computational Fluid Dynamics . . . . . . . . 8

1.3 Cal Poly's CESTOL Effort . . . . . . . . . . . . . . . . . . 9

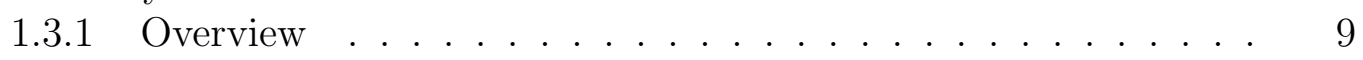

1.3.2 Cal Poly's Wind Tunnel Test . . . . . . . . . . . . . . . 11

1.3.3 Cal Poly Future Work . . . . . . . . . . . . . . . . . . 12

2 Geometry 13

2.1 Adapting Wind Tunnel Model . . . . . . . . . . . . . . . . . . . . . 14

2.2 Engine Geometry . . . . . . . . . . . . . . . . . . 16

2.3 Ciculation Control Wing . . . . . . . . . . . . 17

3 Mesh Generation $\quad 23$

3.1 Meshing Techniques . . . . . . . . . . . . . . . . 23

3.1.1 Surface Mesh Techniques . . . . . . . . . . . . . . . . . . 24

3.1.2 Volume Mesh Techniques . . . . . . . . . . . . . . 26

3.2 Boundary Layer Mesh . . . . . . . . . . . . . . . . . . . . . . . . . . . . . . . . . 28

3.3 Domain Mesh . . . . . . . . . . . . . . . . . . . . 30

3.4 Mesh Refinement . . . . . . . . . . . . . . . . . . . . . . . . . . . 32

3.5 CFD Verification - Grid Convergence Study . . . . . . . . . . . 35 
4 Computational Methods $\quad 37$

4.1 Governing Equations . . . . . . . . . . . . . . . . . . . . . 37

4.1.1 Continuity Equation . . . . . . . . . . . . . 38

4.1.2 Momentum Equation . . . . . . . . . . . . . . . . . . . 38

4.1.3 Energy Equation . . . . . . . . . . . . . . . . . . . . . . . . . . . . . . . . . 49

4.2 Turbulence Modeling . . . . . . . . . . . . . . . . . . . . . 40

4.2.1 Boussinesq Approximation . . . . . . . . . . . . . . . . . . 40

4.2 .2 Standard $k-\epsilon$ Model . . . . . . . . . . . . . . . . . 41

4.2.3 Realizable $k-\epsilon$ Model . . . . . . . . . . . . . . . . . . . 43

4.2.4 Standard $k-\omega$ Model . . . . . . . . . . . . . . . . 45

4.2.5 Shear Stress Transport $k-\omega$ Model . . . . . . . . . . . . . . 47

4.2 .6 Wall Treatment . . . . . . . . . . . . . . . . . . . . 48

4.3 Blowing Coefficient . . . . . . . . . . . . . . . . 52

4.4 CFD Validation - Turbulence Model Evaluation . . . . . . . . . . . 53

4.4.1 2D Circulation Control Airfoil . . . . . . . . . . . 54

4.4.2 3D Circulation Control Wing Section with Engine Simulator . 56

4.5 Computational Resources . . . . . . . . . . . . . . 58

5 Results $\quad 59$

5.1 Preliminary Results . . . . . . . . . . . . . . . . . 59

5.2 Test Matrix . . . . . . . . . . . . . . . . . . . 60

5.3 Solution Overview . . . . . . . . . . . . . . . . . . 63

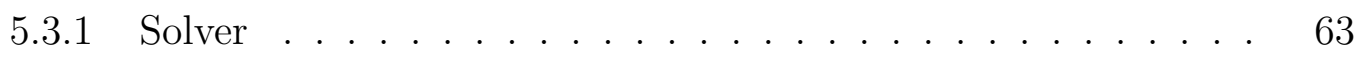

5.3 .2 Wall $y^{+} \ldots \ldots \ldots \ldots$. . . . . . . . . . . . . 64

5.3 .3 Boundary Conditions . . . . . . . . . . . . . . 66

5.3 .4 Convergence . . . . . . . . . . . . . . . . 70

5.3.5 General Solution Characteristics . . . . . . . . . . . 71

5.4 Evaluating Entrainment Performance . . . . . . . . . . . . . . 72

5.5 Solution Details . . . . . . . . . . . . . . . . . . . . . . . . . . . . . . . . 75

5.6 Engine Entrainment Results . . . . . . . . . . . . . . . . 76

5.7 Sensitivities . . . . . . . . . . . . . . . . . . 78

5.8 Engine Deflection Trends . . . . . . . . . . . . . . . . . 80

5.9 QSRA Test Comparison . . . . . . . . . . . . . 81

6 Final Remarks $\quad 84$

6.1 Conclusions ......................... 84

6.2 Future Work . . . . . . . . . . . . . . . 85

$\begin{array}{lr}\text { Bibliography } & 87\end{array}$ 


\section{List of Tables}

4.2.1 Constants for the standard $k-\epsilon$ model as described by White. ${ }^{1}$. . . 43

4.2.2 Constants for the realizable $k-\epsilon$ model as described by FLUENT. ${ }^{2}$. 44

4.2.3 Constants for the standard $k-\omega$ model as described by Wilcox. ${ }^{3}$. . 47

4.2.4 Constants for the shear stress transport $k-\omega$ model as described by

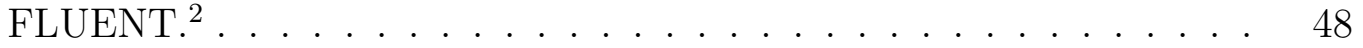

5.1.1 Summary of boundary conditions for proof of concept solutions. . . . 60

5.2.1 Listing of cases solved. . . . . . . . . . . . . . . . . . . . . 62

5.3.1 Test conditions held constant for all runs. ${ }^{*}$ Target $C_{\mu}$ changed in some 63 


\section{List of Figures}

1.2.1 NASA's Quiet Short-Haul Research Aircraft (QSRA). ${ }^{4} \ldots \ldots$. . 3

1.2.2 Circulation control airfoil trail edge with blowing. ${ }^{5} \ldots \ldots \ldots \ldots \ldots$

1.2.3 Circulation Control Wing and Upper Surface Blowing powered-lift con-

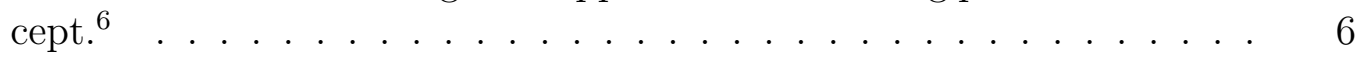

1.3.1 Cal Poly's CESTOL configuration. . . . . . . . . . . . . . . 10

1.3.2 Cross-section of rear circulation control slot and dual radius flap at zero degree flap deflection. . . . . . . . . . . . . . . . 10

1.3.3 Basic wind tunnel model setup. . . . . . . . . . . . . . . . . . 11

2.0.1 A 3-view of Cal Poly's design for the next generation commercial airliner 14

2.1.1 A comparison of the wing-fuselage geometry to the wing blend only geometry. . . . . . . . . . . . . . . . 16

2.1.2 Selected $C_{P}$ distributions for both the wing only and the wing-fuselage solutions . . . . . . . . . . . . . . . . . 17

2.2.1 Turbofan propulsion simulator with the GE90 style nacelle and pylon (left) compared to simplified computational geometry (right). . . . . 18

2.3.1 Wing planform showing important design characteristics. . . . . . . . 19

2.3.2 Cross-section of the wing showing both leading and trailing edge slots. 20

2.3.3 Plenum top view with section cut of the rear plenum. . . . . . . . . 21

2.3.4 Graph that describes the slot height relative to downstream radius and

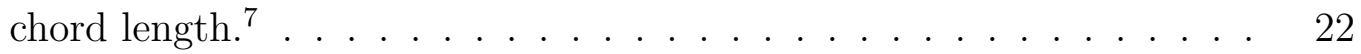

2.3.5 Traditional round trailing edge CC airfoil design (left) versus dual radius flap design (right). . . . . . . . . . . . . . . 22

3.1.1 triangular surface mesh with inset of slot mesh. . . . . . . . . . 25

3.1.2 Two-dimensional representation of the Octree meshing approach. . . . 26

3.1.3 Delaunay criterion applied to a cell. . . . . . . . . . . . . 27

3.1.4 A depiction of first cell created using the advancing front technique on a 2 D surface. . . . . . . . . . . . . . . . . . . 28

3.1.5 Mesh produced by the Octree mesher in ICEM CFD. . . . . . . . 28

3.2.1 Mesh cut plane of the trailing edge slot region. . . . . . . . . . . 29

3.2 .2 Prism mesh on the upper surface on the wing. . . . . . . . . 30

3.3.1 Multi-blocking scheme used to map structured exterior volume mesh. 31 
3.3.2 Hybrid Meshing Concept with domain extents measured in mean geometric chord (MGC) lengths. (Only small section of domain shown) .

3.4.1 Solution with poor resolution in high shear areas. . . . . . . . . . . .

3.4.2 Engine and wing geometry along with orange box where the mesh was refined (left). The original mesh at the engine centerline (top). The refined mesh using the mesh density and the smaller growth rate

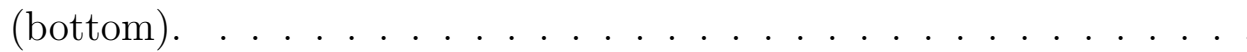

3.4.3 Comparison between the unresolved mesh (top) and the mesh with added density and lower growth rate (bottom). . . . . . . . . . . . 34

3.5.1 Grid independence results using a Richardson's extrapolation. . . . . 36

4.2.1 Non-Dimensionalized velocity plotted versus $y^{+}$shoing subdivisions of nera wall region. ${ }^{2}$.................. . . 50

4.2.2 Wall functions versus near wall approach. ${ }^{2} \ldots . . . . . . .50$

4.4.1 GACC validation airfoil . . . . . . . . . . . . . . 55

4.4.2 Blowing coefficient versus lift coefficient of a circulation control airfoil

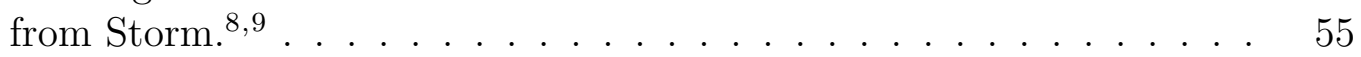

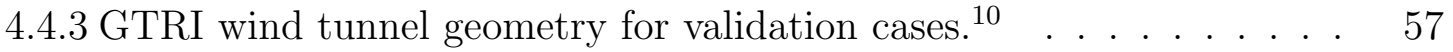

4.4.4 Blowing coefficient versus lift coefficient of a circulation control airfoil from ............................. 57

5.1.1 Preliminary geometry for proof of concept study. . . . . . . . . . . . 59

5.1.2 Mach contours of early entrainment results shown as a proof of concept. 61

5.2.1 CFD test locations located by the bottom trailing edge of the nacelle. 62

5.3 .1 Contours of wall $y^{+}$. . . . . . . . . . . . . . . . 64

5.3.2 Graph of $y^{+}$values. . . . . . . . . . . . . . . . . . . . 65

5.3.3 Engine computational boundary planes and types. . . . . . . . . . 67

5.3.4 Computational boundary comparison for slot geometry. Computational plane modeled at slot exit (left) and computational plane modeled in plenum (right). . . . . . . . . . . . . . 68

5.3.5 Mach contours comparing the two slot modeling approaches. . . . . . 69

5.3.6 Oscillatory behavior of a converged solution. . . . . . . . . . . . . 70

5.3.7 Streamlines showing flow path over the wing. . . . . . . . . . . 72

5.3.8 Streamlines stemming from the flaps showing large vortices. . . . . . 73

5.4.1 Static pressure contours showing separation upstream of slot exit. . . 75

5.4.2 Schematic showing how to measure engine deflection angle. . . . . . . 75

5.6.1 Graph showing engine deflection based on different engine locations. . $\quad 77$

5.6.2 Streamlines showing engine entrainment for different engine heights. . 78

5.7.1 Engine deflection trends fit using cubic spline. . . . . . . . . . . 79

$5.8 .1 C_{\mu}$ sweep for Geometry $6 . \ldots \ldots$. . . . . . . . . 81 


\title{
Nomenclature
}

\section{English Symbols}

\author{
Symbol Definition \\ $C_{D} \quad$ Drag Coefficient \\ $C_{L} \quad$ Lift Coefficient \\ $C_{T} \quad$ Thrust Coefficient \\ $C_{\mu} \quad$ Blowing Coefficient \\ $C C \quad$ Circulation Control \\ $C C A \quad$ Circulation Control Airfoil \\ $C C W \quad$ Circulation Control Wing \\ CESTOL Cruise Effiecient Takeoff and Landing \\ QSRA Quiet Short-Haul Research Aircraft \\ STOL Short Takeoff and Landing \\ USB Upper Surface Blowing

\section{Greek Symbols}

$\begin{array}{ll}\text { Symbol } & \text { Definition } \\ \alpha & \text { Angle of attack, deg } \\ \mu & \text { Dynamic viscosity } \\ \nu & \text { Kinematic viscosity } \\ \rho & \text { Mass density }\end{array}$




\section{Chapter 1}

\section{Introduction}

\subsection{Why is There a Need for Engine Deflection?}

In the early 1980's there was a push to develop high lift aircraft for both military and commercial applications. The research led to a number of investigations in Circulation Control (CC) and Upper Surface Blowing (USB) aircraft to name a few. These developments were researched to reduce the takeoff and landing length of aircraft. In commercial aviation, Short Takeoff and Landing (STOL) aircraft are used to reduce congestion at large airport hubs by allowing bigger aircraft to land at smaller airports. ${ }^{11}$ In military applications these high lift techniques can be utilized to develop aircraft that take off from aircraft carriers or land in small remote landing strips.

It was discovered that coupling the two technologies brought on a tremendous amount of lift. The extra lift came from the circulation control wing entraining the engine thrust downward. The deflected exhaust acted as vectored thrust without having to add complex geometry to do so. Thus engine deflection using a circulation 
control wing was born and a number of research projects have investigated the topic. This paper discusses the possibilities of using an over the wing engine and coupling it with the circulation control wing in order to utilize the same deflection seen using the upper surface blowing engine.

\subsection{Background}

Non-powered and powered lift have been available to aircraft for the last four decades. The argument being one of complexity, feasibility, performance, and application. While non-powered lift has seemingly won the argument over the last half century or so, this paper discusses a number of powered lift options and their application to high lift aircraft.

\subsubsection{Upper Surface Blowing Technology}

Early deflection utilized the Coandã effect by placing the engine over the wing and obtaining some vectored lift in this manner. The Boeing YC-14 and NASA's Quiet Short-Haul Research Aircraft (QSRA) were both experimental USB aircraft that were constructed for STOL applications. Figure 1.2.1 shows the QSRA aircraft with four AVCO-Lycoming YF-102 engines in the upper surface blowing configuration.

USB configurations are considered a reliable high lift concept. USB is the most utilized powered lift concept. USB is popular because the added lift comes at a low research and development cost and can actually reduce complexity when compared to triple slotted flaps with leading edge slats that many aircraft utilize today. The 


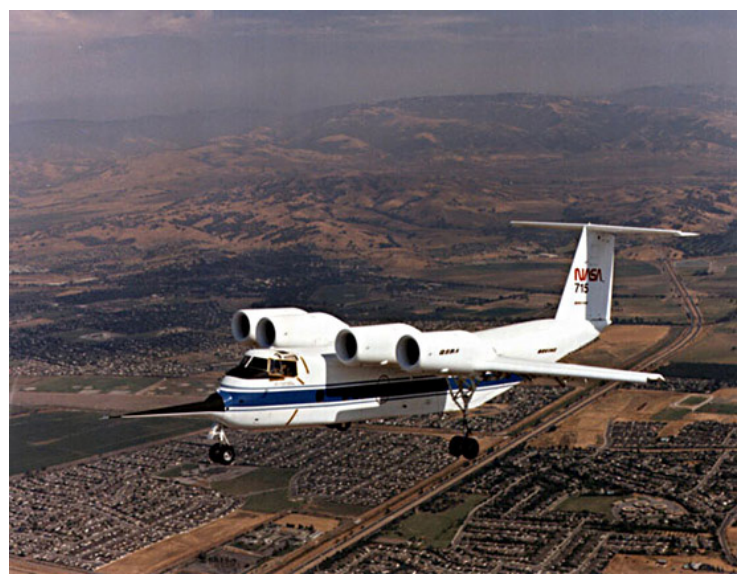

Figure 1.2.1: NASA's Quiet Short-Haul Research Aircraft (QSRA). ${ }^{4}$

shortfalls of this configurations are that it greatly reduces cruise efficiency because the engines add significant scrub drag in flight.

The additional lift generated by the upper surface blowing engine is quickly lost when the engine is moved off the upper surface of the wing. ${ }^{12}$ Some investigation into using an Over The Wing (OTW) pylon mounted engine was made by Coe. ${ }^{13}$ Coe observes that scrub drag in cruise could be reduced by pylon mounting the engine over the wing. But, by pylon mounting the engine almost all the lift benefit from the Conadã effect is lost. Coe remedies this by utilizing a hood to deflect the thrust downward onto the upper surface of the wing in takeoff and landing and retracting the hood in cruise operation. This adds some mechanical complexity but allows the aircraft to maintain some STOL effect while maintaining a more efficient cruise configuration.

USB not only takes advantage of the aerodynamic benefits of mounting a engine over a wing it also provides noise shielding from the ground below. This added benefit 
is a welcomed secondary effect because many times the smaller airports (very same airports high lift aircraft are being used to alleviate congestion at large hubs) are located in urban environments and have strict noise pollution levels. ${ }^{11}$ The added power cannot increase the noise pollution around the airport, thus new advances in noise reduction need to be developed. Noise shielding is one way to deal with the added noise pollution.

\subsubsection{Circulation Control Technology}

Currently most subsonic transport aircraft utilize mechanical flaps to augment maximum lift for takeoff and landing circumstances. These systems allow large aircraft to obtain the high lift needed to takeoff and land in shorter fields. The downfall of these systems is that they can be heavy and mechanically complex. Today many commercial airliners utilize the triple slotted fowler flaps with leading edge slats. The system while effective and reliable is complex and heavy. In the 1970's serious research into circulation control technology exploded with the promise of even higher lift coefficients with less mechanical parts. ${ }^{14,15}$

Circulation control can be applied to many applications including helicopter rotors, submarines, boats, and semi trucks. ${ }^{16}$ The application that applies to this paper and perhaps the most promising is circulation control wings (CCW). CCW's utilize high momentum blowing slots at the trailing edge to increase the effective camber of the airfoil. Traditional CCW's used a tangential blowing slot over the upper surface of a rounded trailing edge as shown in Figure 1.2.2. 


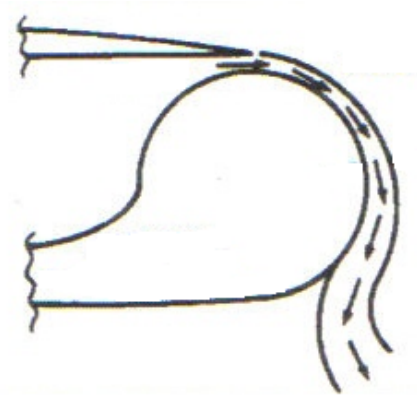

Figure 1.2.2: Circulation control airfoil trail edge with blowing. ${ }^{5}$

The slot blows high momentum air over the rounded trailing edge of the airfoil. A circulation control airfoil works by increasing the effective camber of the airfoil section. The pressure distribution on the airfoil is greatly modified by the added energy to the system. This moves the stagnation point and greatly increases the effective lift when done properly. The technology quickly spread and applied to a wing surface with the hopes of dramatically increasing lift coefficient.

In the late 1970's and Early 1980's a number of flight tests were performed to prove this concept flight worthy. A Grumman A-6A aircraft was modified to include a circulation control wing. ${ }^{17}$ These flight tests showed that the aircraft was flight worthy and that some augmentation was achieved in both static and high altitude flight along with reduction in takeoff and landing distance. The promise and proof of concept for a $\mathrm{CCW}$ was shown during these flight tests. The flights were able to reduce landing distance up to $40 \%$ and high altitude $C_{\text {Lmax }}$ 's in the 3.5 range. This laid the groundwork for Englar to show that a USB in connection with a CCW could prove to be an extremely high lift vehicle that would surpass the lift ability of the 
two concepts individually. ${ }^{6}$

\subsubsection{Engine Deflection}

In the early 1980's NASA modified the USB QSRA to have a CCW to measure the coupling effect of having both lift-generating devices working together. ${ }^{18,19,20}$ The added benefit was that the CCW modified the USB engine flow to deflect it downward such that even greater lift augmentation was achieved. A cross-section of the test setup is shown in Figure 1.2.3.

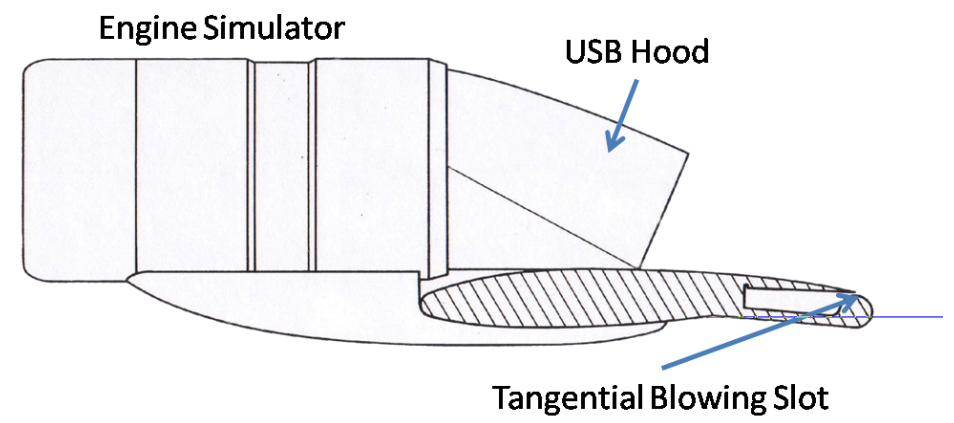

Figure 1.2.3: Circulation Control Wing and Upper Surface Blowing powered-lift concept. ${ }^{6}$

Englar et. al. ${ }^{6}$ looked at the coupling Circulation Control (CC) and USB. Englar showed very promising results for being able to entrain engine flow from the USB engine to deflect downward such that there is a resulting vertical thrust component that can significantly increase the $C_{L}$ at takeoff and landing. There becomes an issue when the engine flow contains too much energy and thus deflection is reduced. For this reason Eppel et. al. showed that lowering the thrust coefficient, $C_{T}$, in order to obtain better thrust deflection angle. ${ }^{20}$

A number of static ground tests were run on this geometry to provide a proof of 
concept. In the tests engine deflection angles ranged from $40-90^{\circ} .20$ In later tests some geometry changes were made that allowed for thrust to be entrained up to $102^{\circ} .^{21}$ These numbers are quite impressive but the high thrust deflections were made under idealized conditions and would not necessarily be achieved in real world circumstances.

One of these idealized conditions being the non-dimensional momentum coefficient of the slot not being a real value in a static test. Typically the momentum coefficient out of the slot is defined by Equation 1.2.1. ${ }^{8}$

$$
C_{\mu}=\frac{\text { Thrust }}{q_{\text {inf }} S_{r e f}}
$$

The key being that the momentum coefficient is non-dimentionalized by dividing thrust by freestream dynamic pressure $\left(q_{i n f}\right)$. In the static tests, such as the ones discussed above, without any freestream flow the slot has a theoretical infinite momentum coefficient. This allows for the engine thrust to be deflected much easier because there is less momentum keeping the flow from turning with the slot flow.

Secondly the thrust rating out of the engine for the high deflection angles is around $25 \%$ of full power. The deflection angle ranges drop off to $30-42^{\circ}$ when the engine is at full power. ${ }^{20}$ The engine deflection increased by $10-22^{\circ}$ over the USB only engine configuration (no blowing).

Lastly special shaped engine hoods and fences better the performance of the apparatus. The fences act as endplates and are added to increase the turning performance 
of the test even if such modifications were not viable for flight applications. The Dshaped nozzle spreads the flow over the entire span of the CCW in order to decrease the engine exhaust momentum for better turning performance. The added fences limited losses at the ends of the circulation control slots which essentially made the problem 2D and eliminated some of the 3D losses.

\subsubsection{Brief History of Computational Fluid Dynamics}

Computational fluid dynamics (CFD) is the study of using numerical methods to solve fluid mechanics problems. Fluid mechanics itself is the study of fluids and how they react under a certain set of internal and external properties. Before the advent of computers capable of processing the millions of calculations required to perform CFD, fluid mechanics problems were typically solved using analytical models and experimental analysis. The theory of CFD existed on paper as far back as the 1700's, but it was theory until computers advanced into the powerful machines they are today. Only in the last two decades has the computer processing power been available to solve complex 3D geometries outside of specialty research labs. With the computer power the computational side of fluid mechanics can enhance our overall ability to analyze fluid mechanics.

Writing CFD codes, even simple codes can be a challenge and is unrealistic for the average CFD user. Today there are numerous academic and commercial CFD codes available. Most users tend to utilize the commercial or academic codes for time and sanity reasons. 
There are many advantages to using CFD as a tool for analyzing fluids problems. Developing wind tunnel models of many different geometries and running different wind tunnel tests can be very expensive and time consuming. CFD requires time, but a large savings can be realized before running wind tunnel tests.

\subsection{Cal Poly's CESTOL Effort}

\subsubsection{Overview}

As part of the NASA initiative to create a Cruise Efficient Short TakeOff and Landing (CESTOL) aircraft, it would be essential to employ some of the benefits of the CCW and USB while being able to reduce the scrub drag at cruise. To achieve this it was apparent that the Cal Poly Team would want to change to an Over the Wing (OTW) mounted engine in order to reduce the drag in cruise. This concept was

previously researched by Coe. ${ }^{13}$ He investigated over the wing blowing (OTWB) by deflecting the exhaust of the engine downward over the wing. This was done using a retractable hood that can be deflected down in takeoff and landing and retracted for cruise performance. This resulted in an increase in circulation lift for relatively small $C_{\mu}$ 's. Without any CCW slots the OTW engine achieved very similar results to more traditional USB engine. Due to the added complexity of this deflection hood it was not used and instead the location of the engine would be optimized to utilize the CC slots to entrain the engine exhaust downward. The resulting configuration is shown in Figure 1.3.1. 


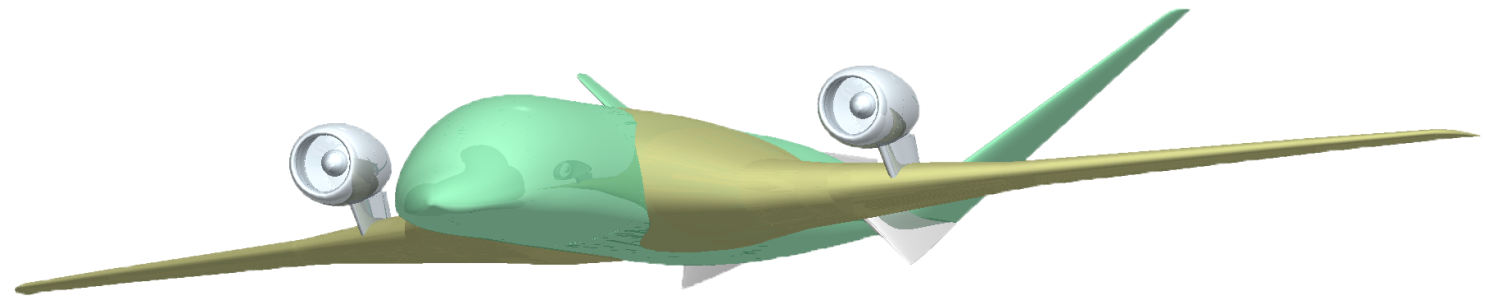

Figure 1.3.1: Cal Poly's CESTOL configuration.

Another significant downfall in early CCW design for an efficient cruising aircraft was the rounded trailing edge airfoil that causes a recirculation zone behind the airfoil and thus an increase in pressure drag. This issue has been addressed and is currently being optimized in Cal Poly's airfoil design. ${ }^{22}$ The flap and CC proposed contains a sharp tailing edge and a CC slot that can be closed during cruise. An example the flap and CC cross-section is shown in Figure 1.3.2 which contains a supercritical airfoil and a dual radius flap design.

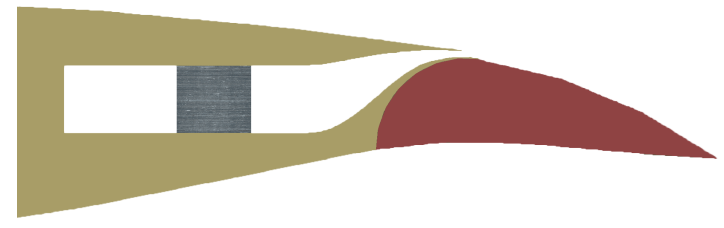

Figure 1.3.2: Cross-section of rear circulation control slot and dual radius flap at zero degree flap deflection.

This CC airfoil configuration also provides better jet thrust recovery and is based on a dual radius flap design. ${ }^{23}$ The dual radius also benefits the CC during blowing conditions because it allows the flow to stay attached longer by changing to a larger radius curve further down the flap where there is momentum loss. Note there is a similar slot at the leading edge blowing air up and over the top surface of the airfoil 
for added lift under takeoff and landing conditions.

\subsubsection{Cal Poly's Wind Tunnel Test}

Cal Poly's effort includes a 10 foot wing span model named AMELIA (Advanced Model for Extreme Lift and Improved Aeroacoustics) that will be tested in NASA's NFAC wind tunnel starting in August 2011. The AMELIA model includes leading/trailing edge blowing slots and turbofan propulsion simulators. The results of the tests will serve as a validation of the configuration and as a collection of wind tunnel tests to compare with CFD results. All of the data will be available to the public as part of Cal Poly's effort to share intellectual knowledge.

The geometry for the wind tunnel model is shown in Figure 1.3.3. This is the actual model setup for the future wind tunnel test and is not the model used for the preliminary results. For the final validation effort, this is the model that will be used.

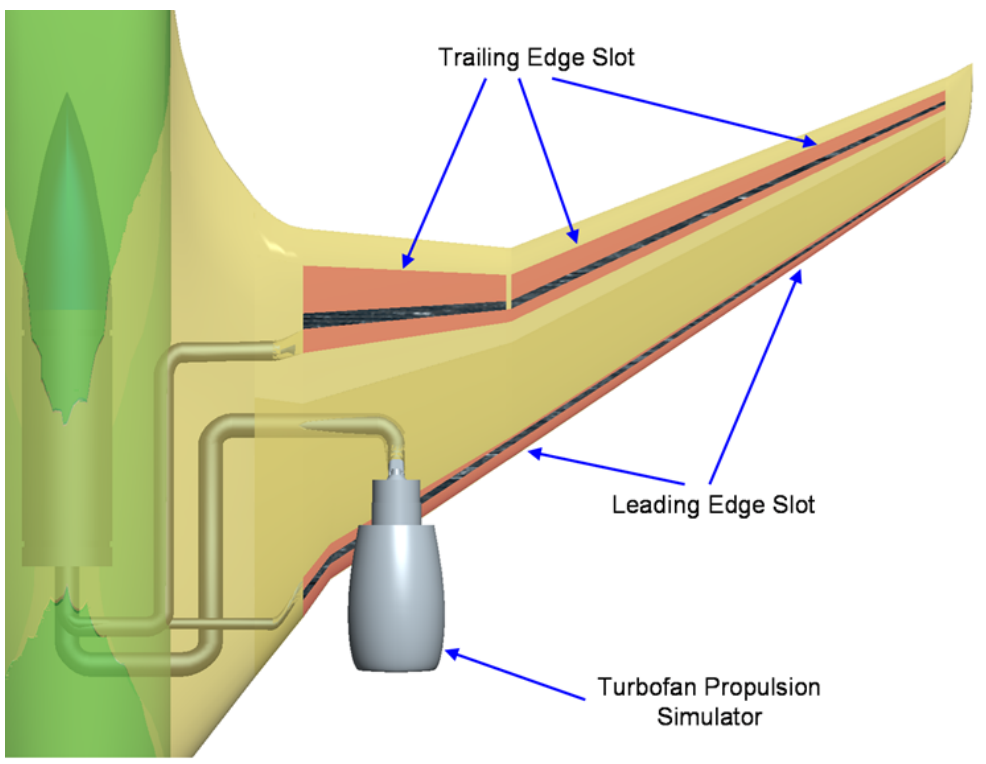

Figure 1.3.3: Basic wind tunnel model setup. 


\subsubsection{Cal Poly Future Work}

Cal Poly's extensive effort to evaluate a new hybrid-wing-body concept and validate/evaluate current CFD tools is far from over. The wind tunnel model is currently finished and being instrumented for its upcoming test. The goal of the wind tunnel test is to provide data on the configuration design and serve as validation data for CFD codes. The geometry, tunnel test conditions, and test results will be open to the public.

Over the last two years the Cal Poly team has developed the skills and CFD knowledge to solve complex 3D CFD solutions of full aircraft configurations. The major shortcoming of CFD has been identified as turbulence modeling prediction. The $v^{2}-f$ turbulence model has been developed and implemented in FLUENT by the Cal Poly team. The model provided some improvement over the standard turbulence models currently in FLUENT More research into developing better turbulence models that will more accurately predict CFD problems such as circulation control wings is being efforted. 


\section{Chapter 2}

\section{Geometry}

The Geometry for this study is based on the wind tunnel model for Cal Poly's upcoming wind tunnel test. Thus, the dimensions of the computational model are in reference to the 1:11th scale wind tunnel model size. This was done because a very detailed and high quality model had been designed for the test, so it provided a good starting point for the geometry for this study. As an added bonus the boundary conditions for the engine simulator and circulation control slots are known for this model. The hybrid-wing-body (HWB) aircraft model contains a circulation control wing $(\mathrm{CCW})$, over the wing (OTW) mounted engines and a complex five surface tail. It became apparent early on that the model was a too complex to use the current computer resources available so there was going to need to be changes to the model to keep the meshes under the maximum size limit of approximately 40 million cells. This limit is due to computational resource constraints. A simple 3-view of the geometry is shown in Figure 2.0.1. The model is highlighted by the hybrid wing planform, the pylon mounted engines that are modeled after the turbofan propulsion simulators 
(TPS), and the V-tail with structural strakes and a dorsal surface.

\begin{tabular}{|c|c|c|}
\hline Wingspan & 10.0 & $\mathrm{ft}$ \\
\hline Length & 9.7 & $\mathrm{ft}$ \\
\hline Height & 2.0 & $\mathrm{ft}$ \\
\hline
\end{tabular}
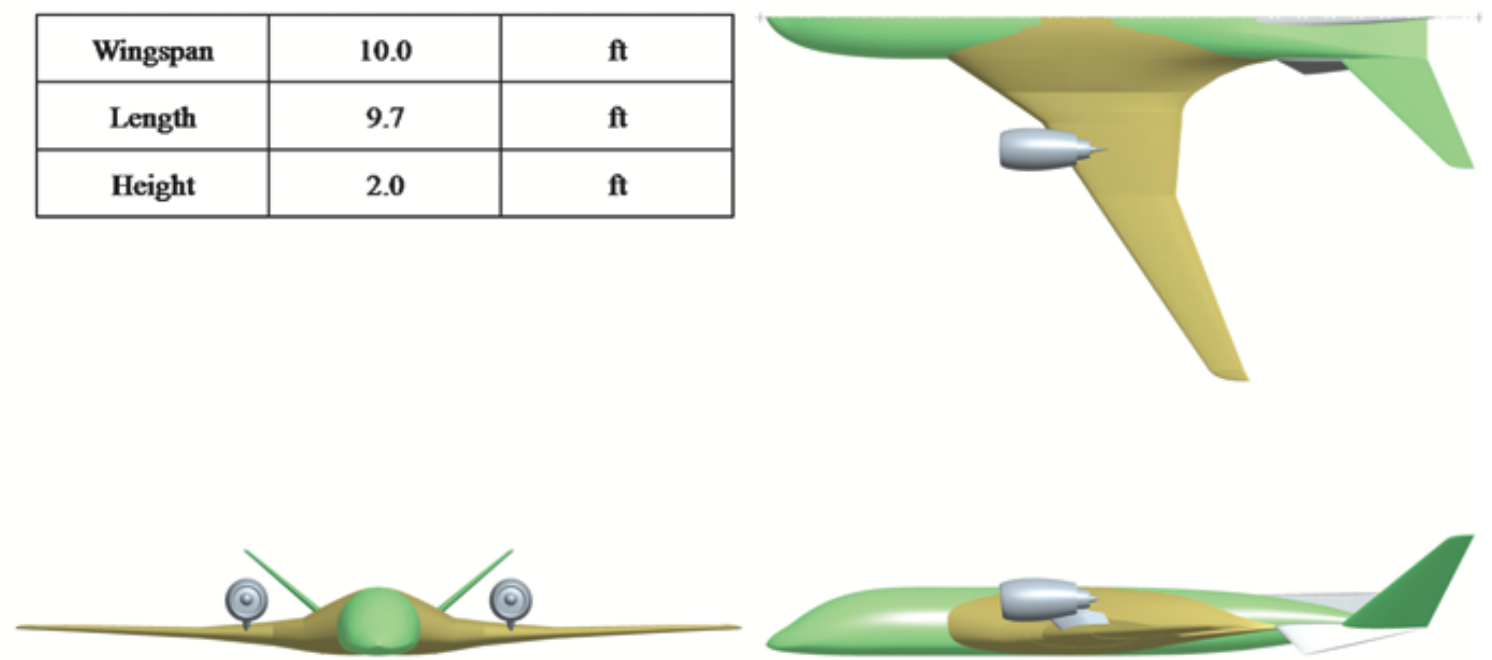

Figure 2.0.1: A 3-view of Cal Poly's design for the next generation commercial airliner

\subsection{Adapting Wind Tunnel Model}

When looking for areas to reduce cell count, the first region of interest was the tail. The tail required a large amount of cells because it has a lot of surface area and a number of very detailed rounds and seams. This required many cells to resolve this area. Since controllability is of no interest in this study and its aerodynamic influence is likely very low, the tails were removed from the geometry. The engine deflection trends observed with and without the tail should be the same. While this was not quantitatively shown because of the aforementioned cell count issues a qualitative look at the problem would lead you to believe that the tail would carry very little impact on the problem at hand.

The next area of interest was the pylon connecting the engine to the wing. This is 
an issue because the study requires that many engine locations are analyzed to find the performance of the aircraft under changing engine locations. Thus, a new pylon would need to be designed for each location. This adds many more variables into the study. In order to eliminate unnecessary variables and to lower the cell count the pylon is removed and a "floating engine" will be used. This concept has been used before in computational studies; Fujino and Kawamura used this to design the location of the engine for the Honda Business Jet. ${ }^{24}$ They studied the location of the engine that best suited them for cruise conditions and were successful in comparing the floating engine computational results to wind tunnel results.

The fuselage is also an area that was explored in order to reduce cell count. A side by side comparison of the wing and fuselage and is shown in Figure 2.1.1. Since the model is a HWB aircraft the wing blend extends to the centerline of the geometry. This allows the user to use a common symmetry plane on both geometries.

In order to validate that the fuselage has little effect on the overall pressure distribution a comparison of the two was made at the same takeoff conditions. Both planforms were run at the same Mach number of 0.15 , which is the fastest takeoff condition to be run during the Cal Poly wind tunnel test. All of the solutions for the engine deflection study will be run at a lower Mach number than the max test condition. A comparison at different $C_{P}$ locations is shown in Figure 2.1.2.

The slice closest to the centerline of the aircraft shows slight deviation at the trailing edge of the airfoil, but at the slice at the engine center line shows the $C_{P}$ 


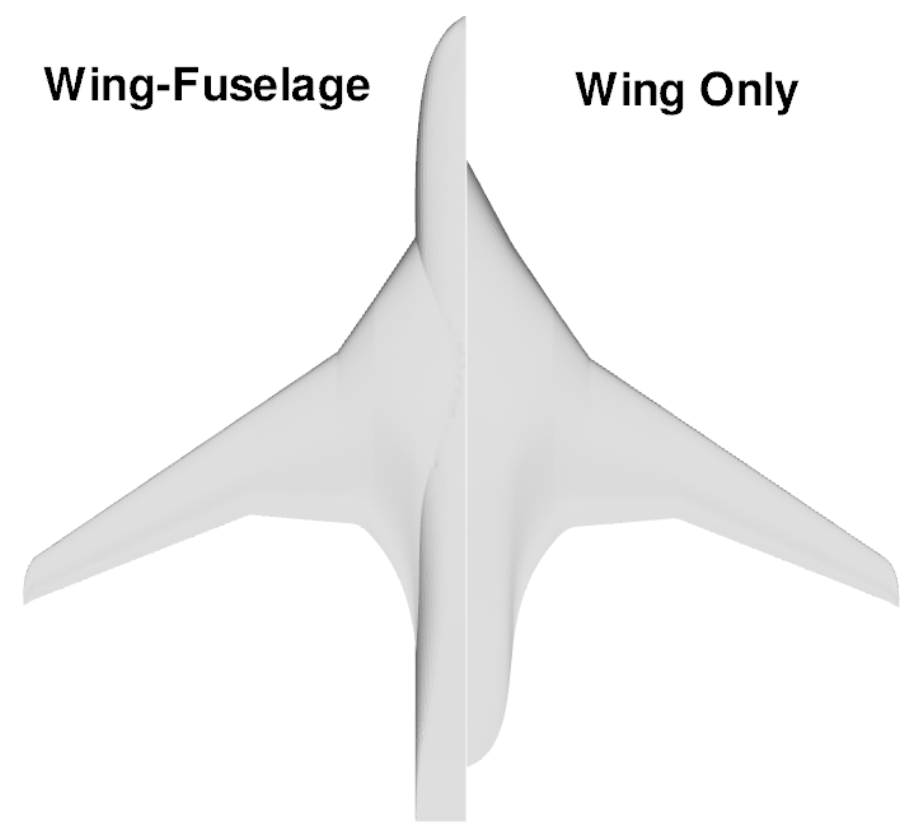

Figure 2.1.1: A comparison of the wing-fuselage geometry to the wing blend only geometry.

distributions are virtually identical. This small deviation near the fuselage intersection is considered very small when examining the high $C_{L}$ 's produced in this problem when the CCW slots are turned on. Thus the fuselage is removed from the geometry for cell reduction purposes. All of the geometry changes combined reduced the cell count by approximately 15 million cells and allowed for refinement in high shear areas such as slots and in the engine wake.

\subsection{Engine Geometry}

The engine is modeled after the TPS unit to be used in the wind tunnel test. The nacelle is modeled similar to the GE90 nacelle used on the Boeing 777. Although the GE-90 engine is a much larger engine, the nacelle was scaled down and some modifications were made so that it was compatible with the propulsion simulator to 


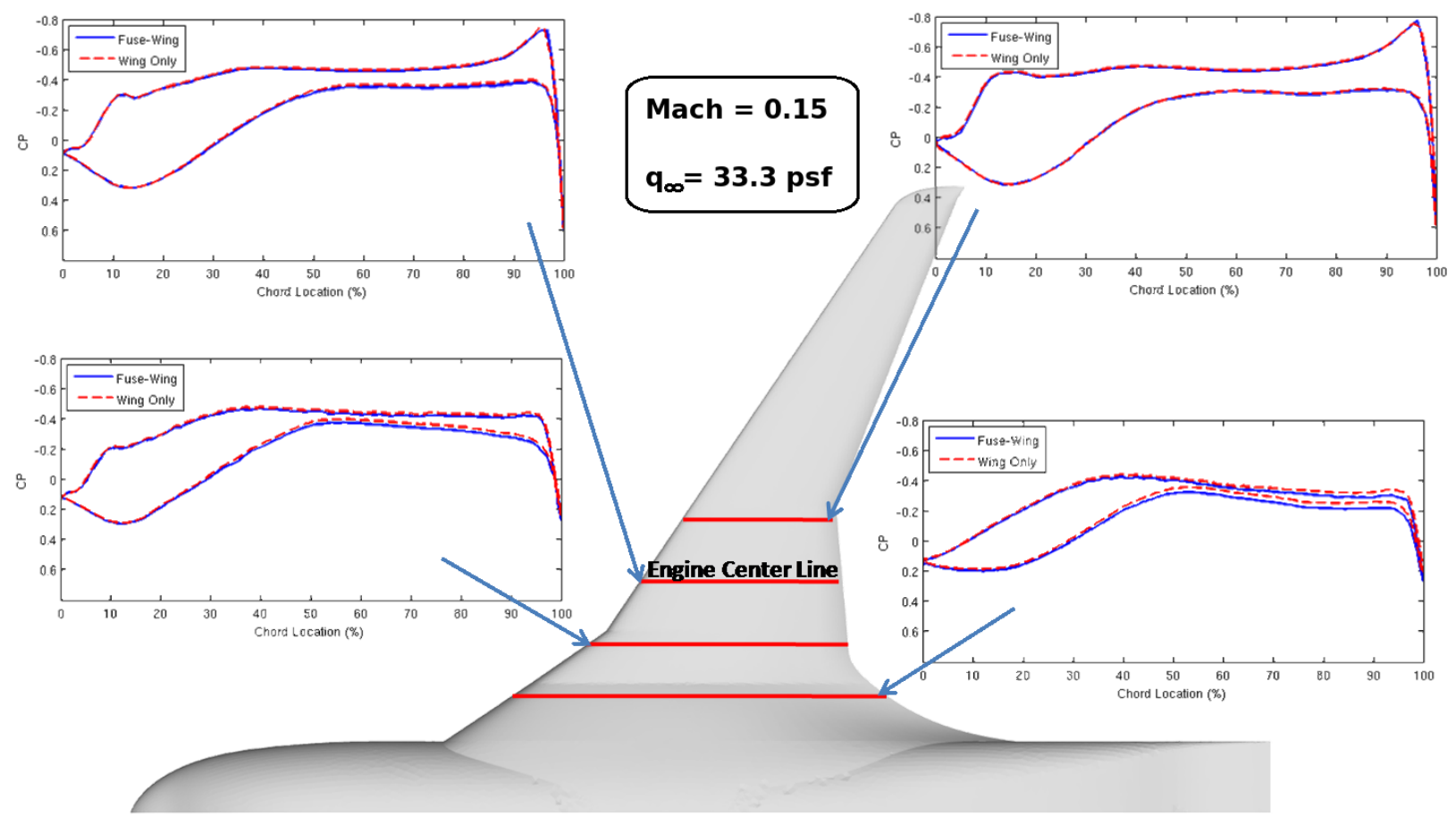

Figure 2.1.2: Selected $C_{P}$ distributions for both the wing only and the wing-fuselage solutions

be used. The simulator itself is a dual flow engine where there is bypass air and core air that provide propulsion. The core is powered by a high pressure air system provided by the tunnel, and the fan is driven by the high pressure air in the core. Since the conditions are known at all stages of this process, this provides information when it becomes time to set the computational boundary conditions. The baseline engine and pylon along with the simplified computational geometry are compared in Figure 2.2.1.

\subsection{Ciculation Control Wing}

The wing, for obvious reasons, was the primary focus of this study. The leading and trailing circulation control slots provided many challenges for geometry creation, 


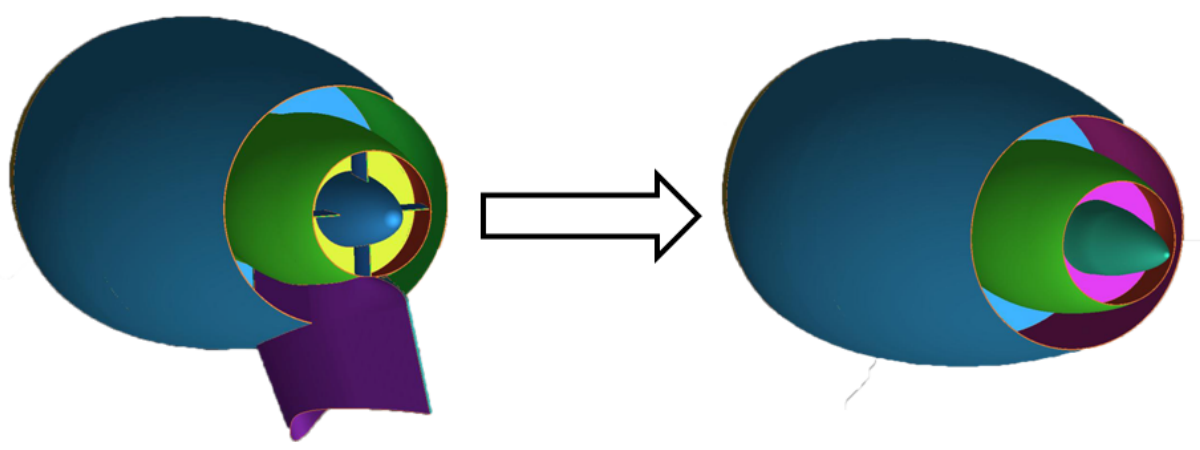

Figure 2.2.1: Turbofan propulsion simulator with the GE90 style nacelle and pylon (left) compared to simplified computational geometry (right).

meshing methods, and computational boundary conditions. The wing itself is a highly swept wing with three distinct wing sections. The first section (closest to the centerline of the geometry) is a wing blend. The blend is a thick root section that blends the wing into the fuselage. The second section, or inboard section, is a highly tapered section that tries to maintain close to a constant trailing edge. The last section separated with a kink at about \%50 span location is a typical outboard wing section. While the sweep is maintained for both inboard and outboard sections, the taper changes drastically. The planform is shown in Figure 2.3.1. The planform was designed by David Hall at DHC Engineering Inc and is described in detail in Cal Poly's CESTOL report. ${ }^{7}$

The most difficult part of this geometry to model, mesh and solve are the relatively small height of the leading and trailing edge circulation control slots. The slots heights are a function of a optimal height to chord ratio. The large fineness ratio between slot height to span causes for very large changes in refinement characteristics. The 


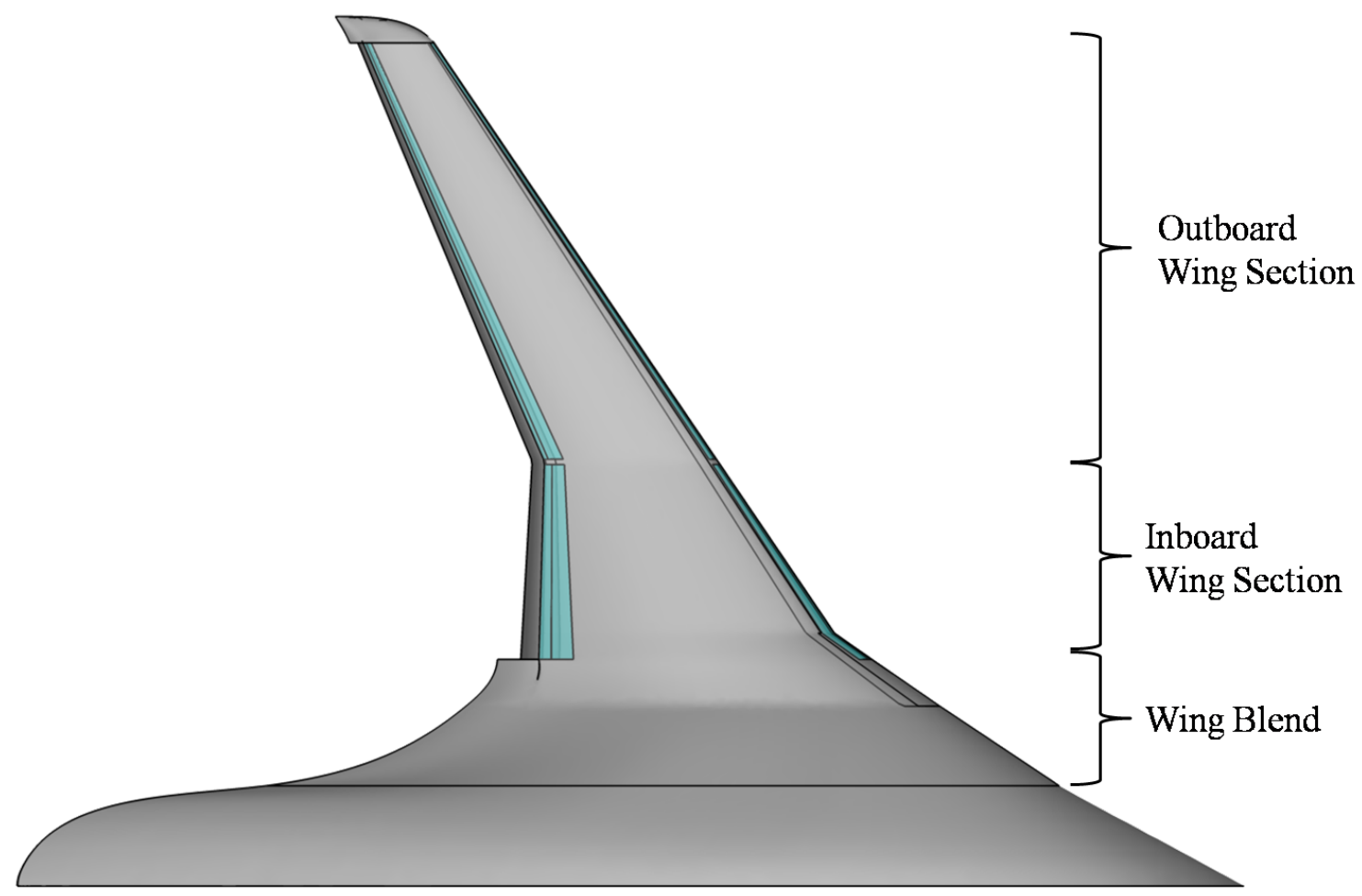

Figure 2.3.1: Wing planform showing important design characteristics.

outboard slot height is approximately 10 thousandths of an inch or about 3 sheets of paper thick. Compared to the wing span of 10 feet, the ratio of the largest feature to smallest feature is about 12000 to 1 . This not only makes it difficult to create solid model, but poses serious challenges in meshing of the geometry. This will be discussed in Section 3.2. A cross-section of the wing showing both leading and trailing edge slots is shown in Figure 2.3.2.

The baseline airfoil chosen for this geometry was the NASA supercritical airfoil SC(2)-0414. ${ }^{7}$ The airfoil was heavily modified at the leading and trailing edges to accommodate the two circulation control slots. The rest of the airfoil shape was held as close as possible to the original geometry. To maximize lift from the trailing edge 


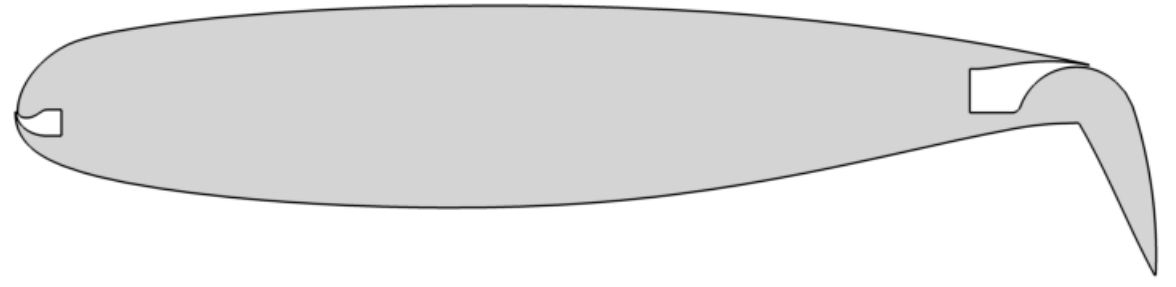

Figure 2.3.2: Cross-section of the wing showing both leading and trailing edge slots.

circulation control slot, the original airfoil flap was enlarged. This larger flap allows the flow from the circulation control slot to influence a larger area on the airfoil thus producing more lift.

As shown in the figure above, the slots are fed through plenums located in both the front and rear of the wing planform. There are four total plenums located two front, two rear, each having a inboard and outboard plenum. The challenge is to come up with an appropriate way to model the slot such that the CFD will provide accurate results. While multiple approaches were attempted which will be discussed in detail in the Boundary Conditions Section 5.3.3, the final choice was to model the plenum back to the where metal foam is placed in the plenum. This provides a surface to set an accurate boundary condition. The plenum layout is shown in Figure 2.3.3.

The slot geometry parameters for each cross-section were specially designed under guidelines provided by Englar and Williams. ${ }^{16}$ The methods were developed for submarines, but were able to be adapted for wing design. The slot height, radius downstream of slot, and chord length ratio are chosen from a graph produced by Englar and Williams. ${ }^{16}$ The graph is reproduced in Figure 2.3.4. 


\section{Light Blue Regions Represent Plenum Locations}

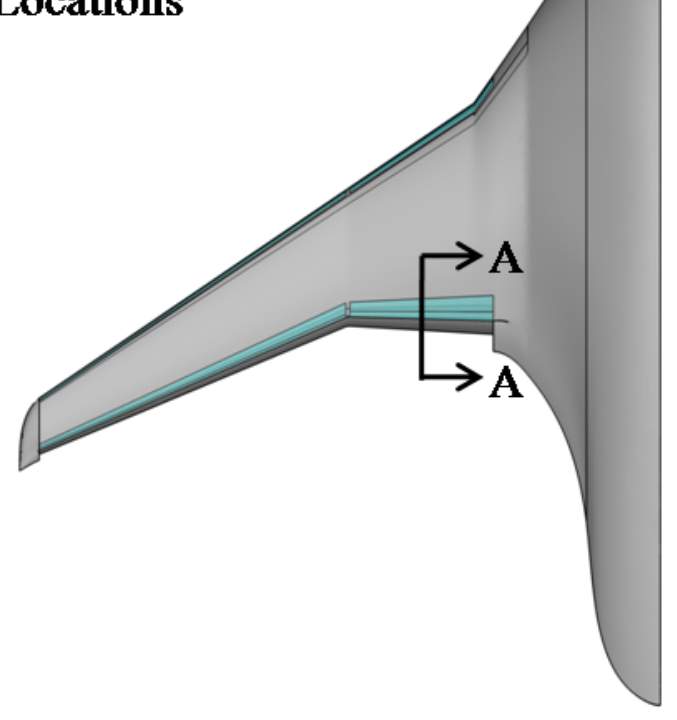

Sec A-A

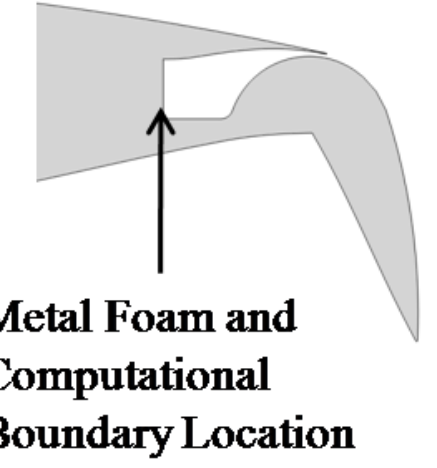

Figure 2.3.3: Plenum top view with section cut of the rear plenum.

The circulation control wing is finished off with a dual radius flap design that allows for higher flap deflections and better cruise performance than a traditional rounded trailing edge. The increase from the smaller to larger flap radius downstream allows for the slot flow to stay attached longer, thus increasing the performance of the circulation control wing. When the flap is retracted for cruise flight the wing performance benefits by not having separation off the larger traditional rounded trailing edge. The dual radius flap is compared to the traditional rounded trailing edge in Figure 2.3.5. 


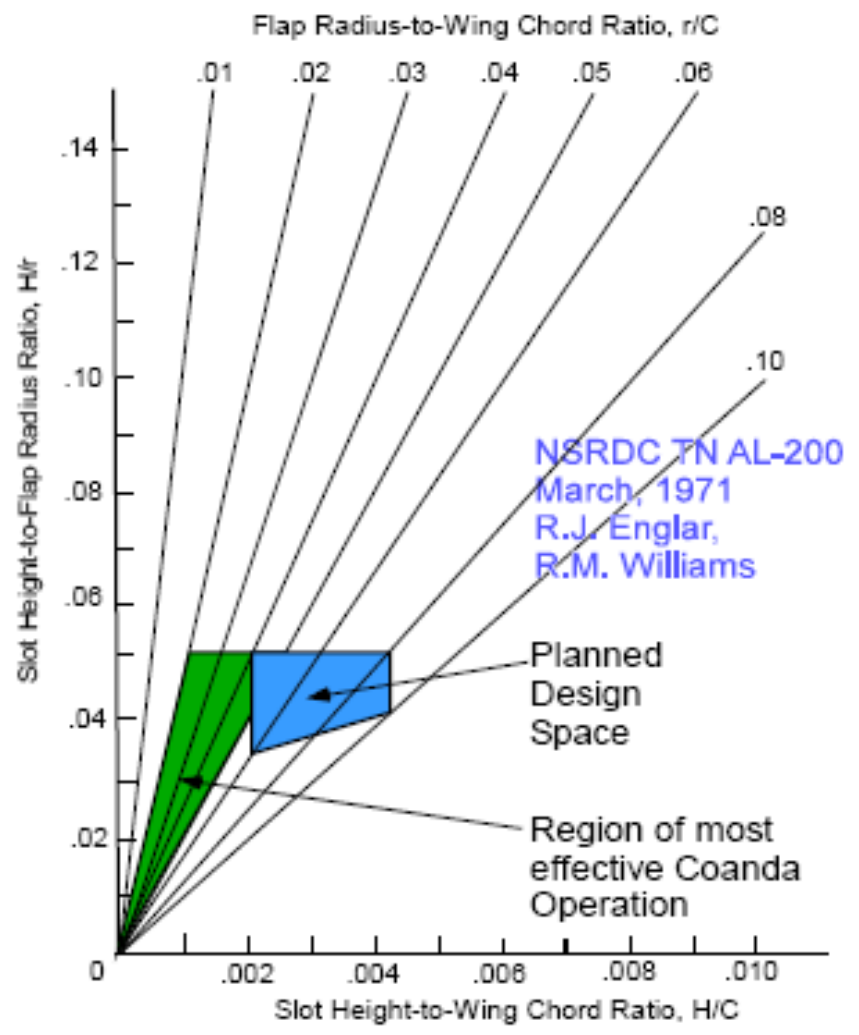

Figure 2.3.4: Graph that describes the slot height relative to downstream radius and chord length. ${ }^{7}$
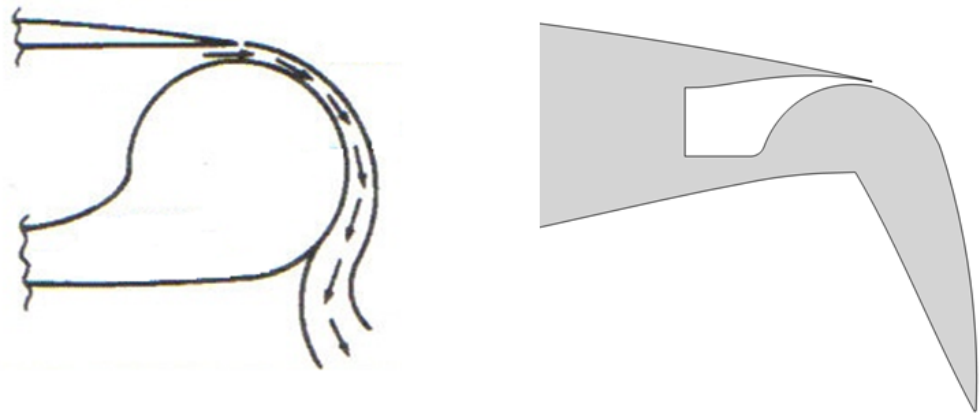

Figure 2.3.5: Traditional round trailing edge CC airfoil design (left) versus dual radius flap design (right). 


\section{Chapter 3}

\section{Mesh Generation}

\subsection{Meshing Techniques}

Grid generation is a process that requires experience, trial and error, and a lot of patience. The grid generation difficulty is compounded when meshing complex geometries, flowfields, or pushing the limits of software. Stuart Connell from GE said, "When computing the flow around complex three dimensional configurations, the generation of the mesh is the most time consuming part of any calculation." 25 The process of obtaining a good mesh on a complex geometry that contains engines, full wing planforms, and circulation control slots provides many challenges.

Meshing simple 2D geometries has been possible for many years, but mesh generation on large complex geometries has been limited due to computational resources. In the last decade, advancements in computational hardware allows for large unstructured meshes to be generated. Without these advancements, it would be impossible to create the solutions for the complex geometry in this thesis. 


\subsubsection{Surface Mesh Techniques}

Surface meshes are typically one of three different types: structured quadrilaterals, unstructured triangles or unstructured octagons. There are other shapes, namely unstructured quadrilaterals that will not be discussed in this paper. Octagons are the base of the polyhedron volume element which are used relatively infrequently. Octagons are great at reducing cell count but solving time is often increased because of the complexity of the cell. Octagons often break down in complex areas such as sharp edges and tight corners. Quadrilaterals can be very good in reducing cell count and for reducing solution error, but the only way to mesh structured quads is to map the entire geometry by hand. Due to the large amount of time it would take to construct a mapped mesh, it was not chosen for this project. For this application, the problem required an unstructured element that defines complex geometry well and is robust enough that it can mesh difficult areas. Difficult areas being areas that have other surfaces in close proximity, such as CCW slots, and areas where there are many intersecting surfaces, such as a wing blend. Triangle elements are best at refining these features which is why they were the method of choice. Part of the surface mesh showing some of the more complex areas of the geometry is shown in Figure 3.1.1

There are two techniques ICEM CFD (the meshing software for this project) provides for surface meshing. The first being a mapped method where the user sets the individual node spacing and size limits on each individual surface. This can be difficult when many surfaces are involved because node spacing on adjacent surfaces 


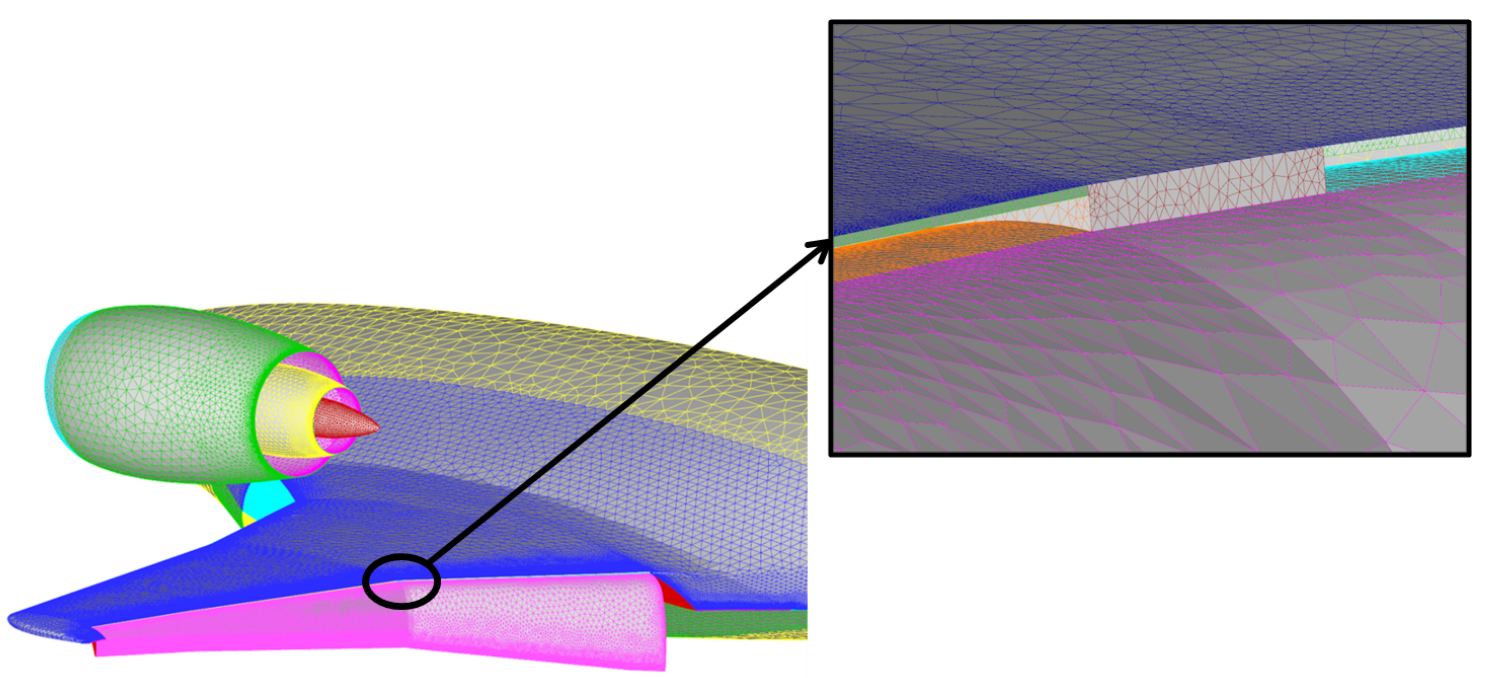

Figure 3.1.1: triangular surface mesh with inset of slot mesh.

must match. When the model involves upwards of 250 surfaces, as is the case for the geometries in this paper, this task is not only very time consuming but daunting to complete.

The primary surface meshing technique in ICEM CFD uses Octree volume meshing scheme to create a complete volume mesh, save the surface mesh, and discard the rest of the cells in the domain. This seems counter-intuitive because if the meshing program has already created the volume mesh with the surface mesh, there is not a lot of reason to use a different volume meshing technique on an Octree developed surface mesh. ICEM CFD's ability to generate quality meshes robustly using the Octree method is the reason that it was chosen as the surface mesher in this project. 


\subsubsection{Volume Mesh Techniques}

The highly complex geometry limits the ability to use a structured type mesh so an unstructured mesh is required. Triangles are the base element for the tetrahedral cells and are the most common volume element used in unstructured meshing today. Tetrahedral meshes have proven over time to be the cell of choice for complex geometries that require unstructured meshes. For this reason, and because ICEM CFD only provides tetrahedrals for unstructured volume meshing, tetrahedrals were chosen to model the geometry.

There are three approaches to generating tetrahedral meshes; Octree, Delaunay, and advancing front. ${ }^{26}$ ICEM CFD provides the option to utilize any one of these. Octree utilizes a method of subdivision and is a top down approach which means it starts with a few big cubes modeling the entire domain. It recursively subdivides the cubes into smaller blocks until it satisfies the specified surface resolution constraints. The method finds the intersections with the geometry and then deletes the portion lying outside the domain of interest. In $3 \mathrm{D}$, the are then converted into irregular polygons and then finally into tetrahedrals. ${ }^{27,28}$ This is illustrated in $2 \mathrm{D}$ in Figure 3.1.2..$^{29}$

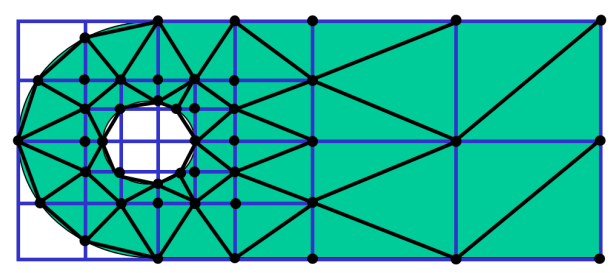

Figure 3.1.2: Two-dimensional representation of the Octree meshing approach. 
Delaunay is the most common approach to volume meshing. ${ }^{29}$ While it has a very simple set of meshing constraints, it does need a starting point to begin the meshing process. Usually, this means providing the meshing program with a surface mesh. Once the program has a surface mesh the Delaunay approach sets the constraint that no node may lie within the circumsphere of another element. This is illustrated in Figure 3.1.3. ${ }^{29}$

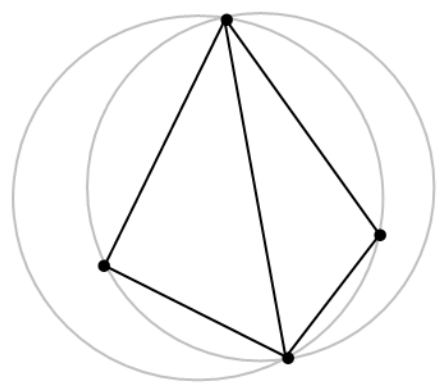

Figure 3.1.3: Delaunay criterion applied to a cell.

Just like the Delaunay method, advancing front requires a boundary mesh as a starting point for the volume meshing scheme. This method starts at the boundaries and tries to work inward by making ideal cells moving into the domain one layer at a time. ${ }^{30}$ This method is a little more robust than Delaunay at handling difficult geometries but it is generally slower and produces lower quality meshes. Figure 3.1.4 depicts the first cell on the first front being constructed. The meshing algorithm attempts to create a perfect cell first and then searches to see if the cell intersects any other nodes. ${ }^{31}$

Octree was the method selected for the geometries in this thesis. While ICEM CFD provides all three methods discussed above the requirement of Delaunay and 


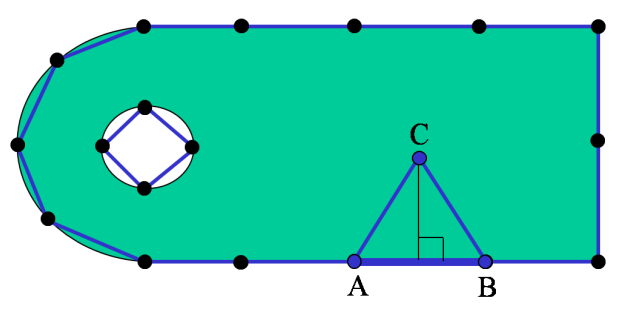

Figure 3.1.4: A depiction of first cell created using the advancing front technique on a $2 \mathrm{D}$ surface.

advancing front adds in an added hurdle of having to provide a surface mesh. Again ICEM CFD has methods to obtain a surface mesh, but most fall well short of the capabilities of other meshing programs. An Octree created tetrahedral mesh cut plane is shown in Figure 3.1.5.

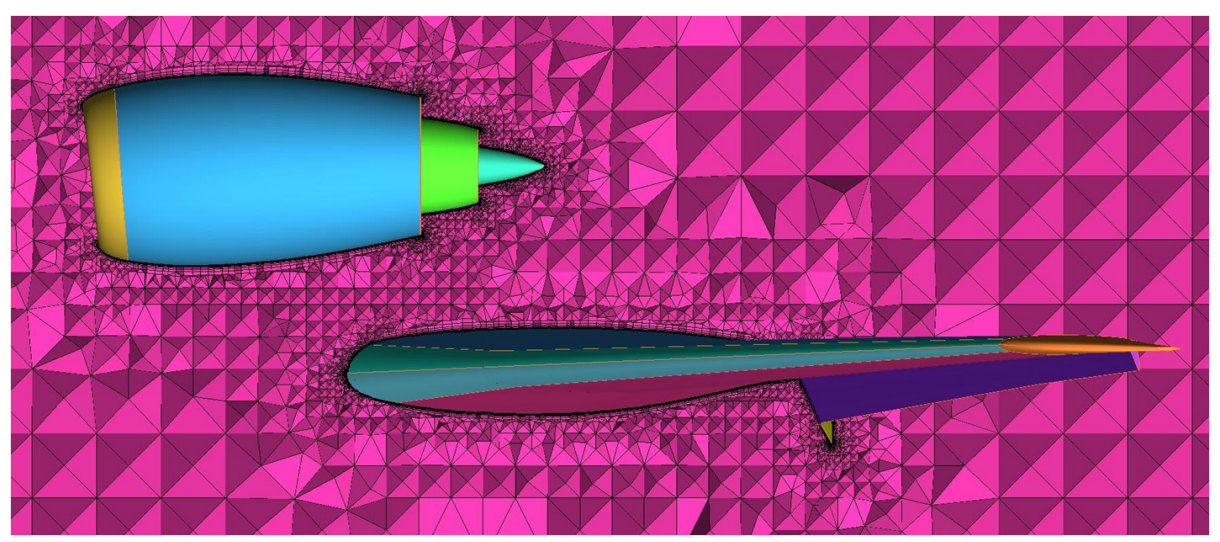

Figure 3.1.5: Mesh produced by the Octree mesher in ICEM CFD.

\subsection{Boundary Layer Mesh}

It is very important to correctly resolve the boundary layer because the turbulence model can be very sensitive to the height and quality of the near wall mesh. Section 4.2.6 discusses the particulars of the near wall treatment for the turbulence models. There are three factors to consider when generating a near wall (prism) mesh: cell quality measured by cell skewness, last prism layer to first tetrahedral 
volume transition and last prism layer distance from centroid to cell wall versus first tetrahedral distance from centriod to cell wall. These are all nice things to consider when generating a mesh, but in applied CFD often concessions have to be made between what the mesher is able to successfully generate versus the three criteria listed above. Due to complex areas, such as the slot area shown in Figure 3.2.1, the prism mesh was first considered solvable if it satisfied tight skewness levels and secondly showed reasonable volume transition.

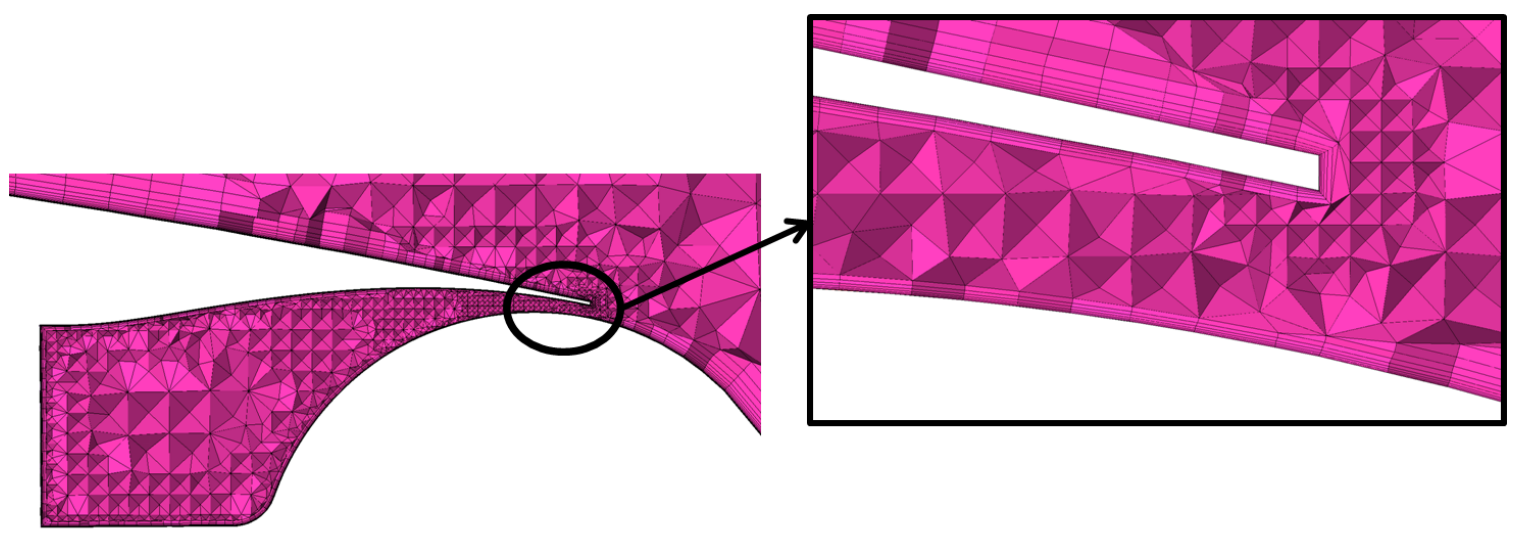

Figure 3.2.1: Mesh cut plane of the trailing edge slot region.

Meshing of the slot was very difficult because it was very small in comparison to the rest of the geometry. The target number of cells in the slot was 16-20 including six prism layers on the upper and lower sections of the slot. The slot shown in Figure 3.2.1 contains 7 prism layers on the upper slot surface, 7 prism layers on lower slot surface and approximately 6 tetrahedral cells between the two layers. The volume transition in the slot was sacrificed due to the mesher having issues resolving the area, but it is still within acceptable limits. In easier mesh areas, the volume transition and quality 
are nearly perfect as is the case in Figure 3.2.2 showing the near wall mesh of the upper surface of the wing.

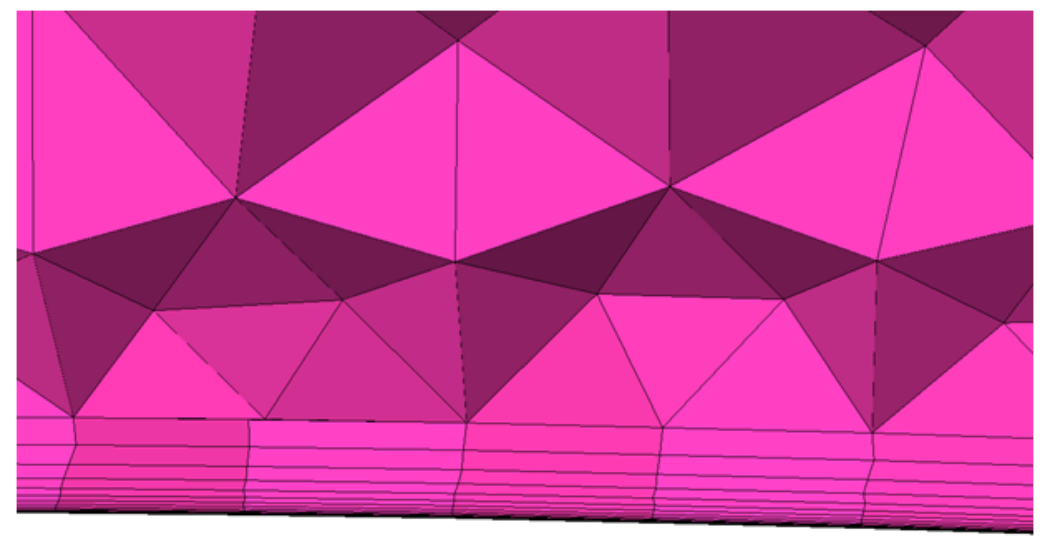

Figure 3.2.2: Prism mesh on the upper surface on the wing.

\subsection{Domain Mesh}

To model the flow field that exists outside the immediate regime that surrounds the aircraft, hexahedral elements proved to be the most efficient way to capture the secondary flow features. The mesh was assembled using a number of structured blocks attached to each other. Using this multi-blocking approach gives the user a lot of flexibility in choosing how to map out each structured block. Perhaps the user wants to refine a certain block but does not need the same grid resolution in a different box. For example, it is expected that the flow field will exhibit secondary flow features such as wingtip vortices. These vortices are known to travel well behind the aircraft before dissipating completely. In subsonic flow, failure to capture this feature accurately may greatly affect the final computational solution. Thus, it would be advised that 
the blocks that exist behind the aircraft be much more resolved. Figure 3.3 .1 shows an example of what the blocking scheme looks like.

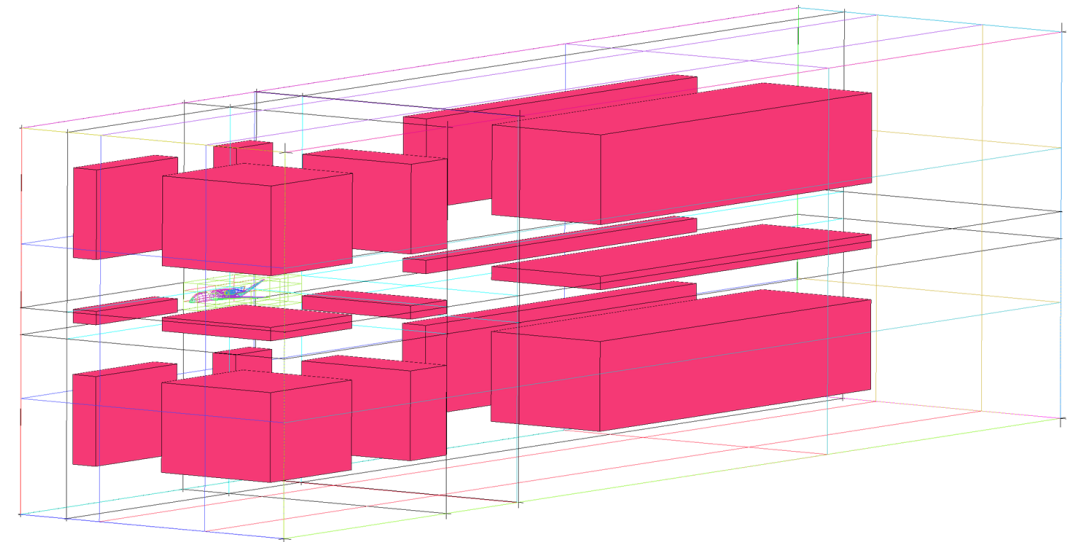

Figure 3.3.1: Multi-blocking scheme used to map structured exterior volume mesh.

Instead of using hexahedral elements, the user could use unstructured tetrahedral elements to model this portion of the mesh. However, doing so would diminish the accuracy of the solution and would unnecessarily increase total cell count. Following general rules of thumb for grid generation, the structured domain was modeled to be roughly 10 mean geometric chord (MGC) lengths upstream, 5 MGC lengths upward, 7 MGC lengths downward and to the side of the aircraft. Downstream of the aircraft, however, structured elements extended out to $25 \mathrm{MGC}$ lengths. An example of the hybrid mesh used is shown for the HWB concept in Figure 3.3.2. 


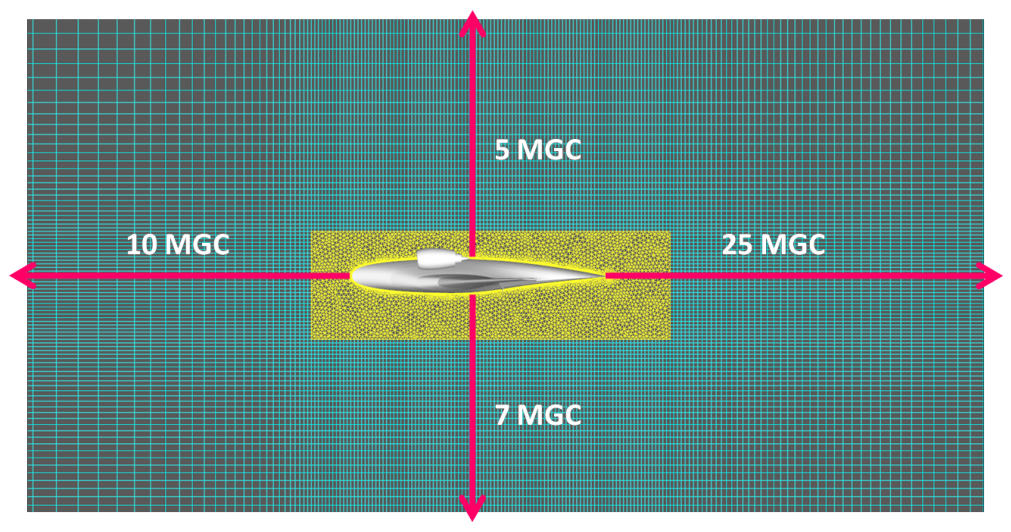

Figure 3.3.2: Hybrid Meshing Concept with domain extents measured in mean geometric chord (MGC) lengths. (Only small section of domain shown)

\subsection{Mesh Refinement}

Early solutions showed that there were regions of the mesh that could be improved to provide more accurate answers. The engine and slot exhaust area in particular were areas that were shown to have distorted shear layers and coarse cell size. Since it is important to resolve these shear layers to correctly predict the flowfield, it was necessary to provide some mesh refinement in these areas. The Mach number contours at the centerline of the engine are shown in Figure 3.4.1.

There were two resolution issues with this particular solution. First, the engine wake was shown to be grossly under refined and second, the entire flowfield was a little too coarse. For the engine wake it would be ideal to resolve the grainy areas of this solution without having to resolve the entire flowfield to the same resolution. The way to do this in ICEM is to use mesh densities which allow the user to set specific mesh settings inside a set volume. A smaller max size was set inside the density to 


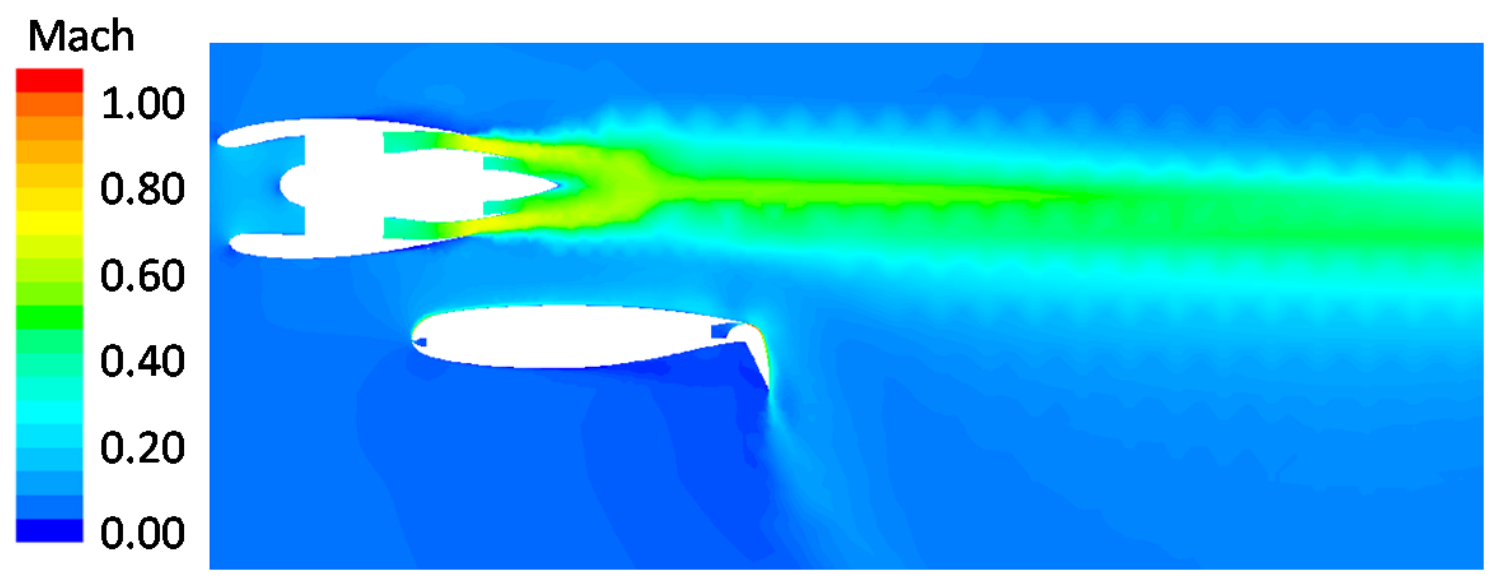

Figure 3.4.1: Solution with poor resolution in high shear areas.

keep an appropriate resolution to the engine wake. To resolve the entire flowfield, the tetrahedral growth rate was lowered from 1.25 to 1.2. The change in mesh along with the mesh density is shown in Figure 3.4.2
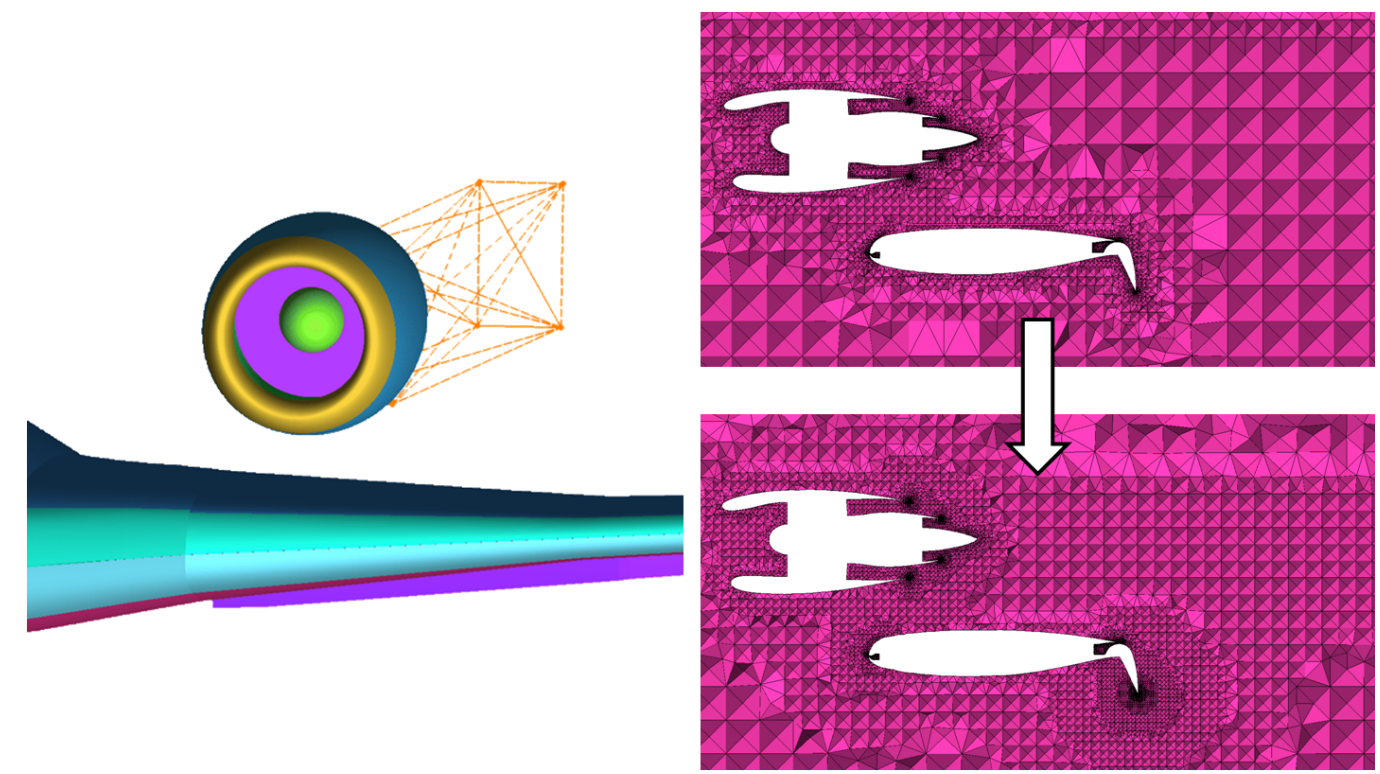

Figure 3.4.2: Engine and wing geometry along with orange box where the mesh was refined (left). The original mesh at the engine centerline (top). The refined mesh using the mesh density and the smaller growth rate (bottom).

This refinement comes at a cost of approximately 5 million cells added to the 
domain. This was deemed necessary to correctly resolve the high shear layers to better the results. A comparison between the two solutions is shown in Figure 3.4.3.

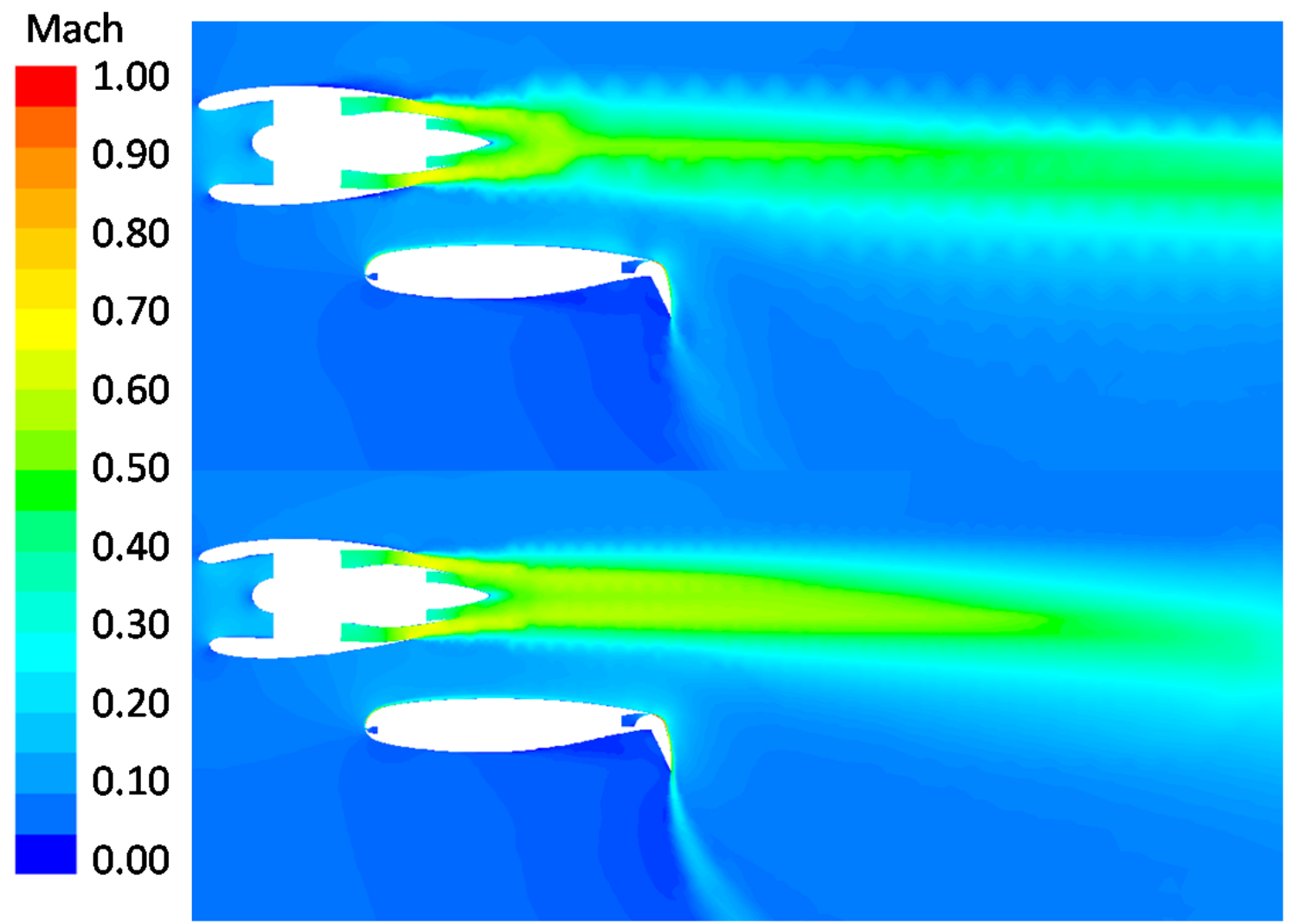

Figure 3.4.3: Comparison between the unresolved mesh (top) and the mesh with added density and lower growth rate (bottom).

The distorted shear layer in the engine exhaust is defined much clearer and is a vast improvement over the earlier solutions. The circulation control jet is much clearer and crisper as the jet hits the freestream. This cleaner solution allows for much easier visualization of the engine deflection. The entire solution qualitatively looks better, but in order to quantitatively measure if the mesh presented is a good solution, a grid independence study needs to be performed. 


\subsection{CFD Verification - Grid Convergence Study}

Experience shows that CFD can produce widely different answers for the same problem when solutions are solved in different manners. One of the mechanisms causing these inconsistencies is mesh refinement. It is important for the user to be able to produce consistent results on different geometries by maintaining consistent meshing practices. One way of ensuring a consistent mesh is performing a mesh independence study. Once a mesh is determined to satisfy a certain criteria, then the same mesh settings are used for all CFD solutions in the work. This is just one of many steps required to ensure the quality of the CFD solutions.

Using a Richardson's Extrapolation, over three different size grids will verify whether or not the grid is fine enough. The method extrapolates to the correct solution based on test points if you could theoretically make the mesh infinitely fine. The blue line in Figure 3.5.1 indicates this theoretically infinitely fine solution. The grid independence method is shown in detail by Celik. ${ }^{32}$ Since this study is a lifting application the lift coefficient is monitored and the extrapolation shows that the extrapolated solution is $0.28 \%$ deviation from the finest mesh of approximately 38 million cells.

The results show good convergence on a refined mesh. The 38 million fine mesh was used for all results shown in this paper. The red dotted lines represent the error bars bounding the fine solution. Overall, the results are encouraging that the mesh is fine enough to measure small changes in lift coefficient. These mesh settings are 


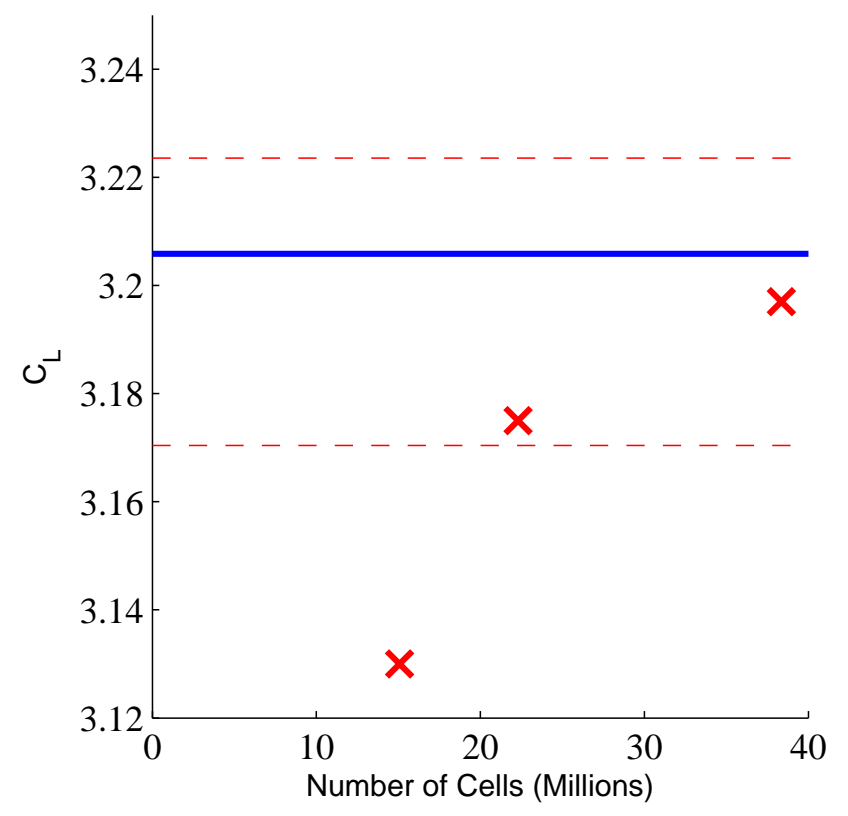

Figure 3.5.1: Grid independence results using a Richardson's extrapolation.

carefully carried over to the rest of the geometries so that consistent results can be produced and thus one solution can be compared to another. Then choices can be made on whether one configuration is better than the other. Even slight inconsistencies in mesh or solution practices can lead to inconsistent results and inaccurate conclusions. 


\section{Chapter 4}

\section{Computational Methods}

FLUENT $^{33}$ was used as the solver for this paper. The numerical method used is a fully implicit second order accurate finite volume method. The Reynolds-Averaged Navier-Stokes (RANS) equations were used in the simulations. RANS equations are advantageous because they provide time averaged solutions to the Navier-Stokes equations. For turbulent flow, such as the flow in this work, it is necessary to provide information about the turbulence. This will be explored later in this section.

\subsection{Governing Equations}

For reader sanity the full derivation of the governing equations will not be provided, but rather a summary of the basics will be presented. For full derivations

feel free to explore Tannehill et. al. ${ }^{34}$ Since FLUENT was used as the solver, the equations presented below come from the FLUENT User Manuel. ${ }^{2}$ 


\subsubsection{Continuity Equation}

Conservation of mass is satisfied by solving the continuity equation. For an inertial reference frame its most general form is written as

$$
\frac{\partial \rho}{\partial t}+\rho \nabla \vec{U}=0
$$

where $\vec{U}$ is the velocity vector. In order to add a source term to compensate for mass added to the system $S_{m}$ is added to the right hand side of the equation.

$$
\frac{\partial \rho}{\partial t}+\rho \nabla \vec{U}=S_{m}
$$

$S_{m}$ accounts for any vaporization or user defined values that are added to the system.

\subsubsection{Momentum Equation}

The momentum equation for a non-accelerating inertial reference frame can be written as $^{2,35}$

$$
\frac{\partial}{\partial t}(\rho \vec{v})+\nabla \cdot(\rho \vec{v} \vec{v})=-\nabla p+\nabla \cdot(\overline{\bar{\tau}})+\rho \vec{g}+\vec{F}
$$

where $p$ the static pressure, $\rho \vec{g}$ is the gravitational body force, $\vec{F}$ is the external body forces, and $\overline{\bar{\tau}}$ is the stress tensor and can be written as

$$
\overline{\bar{\tau}}=\mu\left[\left(\nabla \vec{v}+\nabla \vec{v}^{T}\right)-\frac{2}{3} \nabla \cdot \vec{v} I\right]
$$

where $\mu$ is the molecular viscosity and $I$ is the unit tensor. 


\subsubsection{Energy Equation}

The energy equation can be written as

$$
\frac{\partial}{\partial t}(\rho E)+\nabla \cdot[\vec{v}(\rho E+p)]=\nabla \cdot\left[k_{e f f} \nabla T-\sum h_{j} \vec{J}_{j}+\left(\overline{\bar{\tau}}_{e f f} \cdot \vec{v}\right)\right]+S_{h}
$$

where $k_{e f f}$ is the effective conductivity. $k_{\text {eff }}$ equals $k+k_{t}$ where the latter is the turbulent conductivity added by the turbulence model. $\vec{J}_{j}$ is the diffusion flux of species $j . \quad S_{h}$ is the heat of chemical reaction and includes other volumetric heat sources. The other three terms on the right hand side of the above equation (from left to right) are energy transfer due to conduction, species diffusion, and viscous dissipation.

$E$ from the above equation can be written as

$$
E=h-\frac{p}{\rho}+\frac{v^{2}}{2}
$$

where $\mathrm{h}$ is sensible enthalpy and can be defined as

$$
h=\sum Y_{j} h_{j}
$$

where $Y_{j}$ is the mass fraction of species $\mathrm{j}$ and

$$
h_{j}=\int_{T_{r e f}}^{T} c_{p, j} d T
$$

where $T_{\text {ref }}$ is $298.15 \mathrm{~K}$. 


\subsection{Turbulence Modeling}

A quote from Hinze says "Turbulent fluid motion is an irregular condition of flow in which the various quantities show a random variation with time and space coordinates so that statistically distinct average values can be discerned." ${ }^{36}$ As of today turbulence is accounted for in CFD in one of two ways; first turbulence can be solved via Direct Numerical Simulation (DNS) and secondly a model can be used to approximate the turbulence in the solution. DNS is very computationally expensive due to having to be able to resolve the solution to the length scales of the smallest eddy to the largest feature in the solution. Today DNS can be used on relatively simple geometries at great computational expense. Due to limitations in today's computational resources DNS is not reasonable for solutions of this complex nature. Thus, turbulence models become an important discussion in this work.

\subsubsection{Boussinesq Approximation}

The Boussinesq approximation lays the ground work for many turbulence models by hypothesizing that shearing stress is related to the rate of mean strain through an apparent scalar turbulent viscosity. This is how most common turbulence models approximate Reynolds stress. The Boussinesq assumption give the following

$$
-\overline{\left(\rho u_{i}^{\prime} u_{j}^{\prime}\right)}=2 \mu_{T} S_{i j}-\frac{2}{3} \delta_{i j}\left[\mu_{T} \frac{\partial u_{k}}{\partial x_{k}}+\rho \bar{k}\right]
$$

where $\mu$ is the turbulent viscosity, $\bar{k}$ is the kinetic energy of turbulence defined by 


$$
\bar{k}=\frac{\overline{u_{i}^{\prime} u_{j}^{\prime}}}{2}
$$

where the $u^{\prime}$ are the turbulent fluctuations in the flowfield. The mean strain tensor from above can be described as

$$
S_{i j}=\frac{1}{2}\left[\frac{\partial u_{i}}{\partial x_{j}}+\frac{\partial u_{j}}{\partial x_{i}}\right]
$$

For some flow regimes these calculations have been validated and proven to be a good approximation. It will be assumed that the solutions in this paper will fall into one of these regimes. The models explored and discussed for this work are the standard $k-\epsilon$ model, the realizable $k-\epsilon$ model, the standard $k-\omega$ model and the shear stress transport $k-\omega$ models. Some effort has been made as part of Cal Poly's NASA grant to develop the $v^{2}-f$ model in FLUENT, but it will not be discussed in detail in this paper. All of these models rely on the Boussinesq approximation.

\subsubsection{Standard $k-\epsilon$ Model}

The standard $k-\epsilon$ is one of the most common and robust turbulence models available today. The model is accurate and stable for a large range of flow fields. This turbulence model was attractive because of its wide use and very stable nature of the solution calculations. Two other variants of the two-equation $k-\epsilon$ are available in FLUENT. The renormalization group theory (RNG) $k-\epsilon$ model and the realizable $k-\epsilon$ model make improvements to the performance to the standard $k-\epsilon$ version. 
The RNG $k-\epsilon$ model will not be discussed in this paper but the realizable $k-\epsilon$ will be explored later.

The $k-\epsilon$ model assumes that the flow is fully turbulent. Recent developments in FLUENT allow for transition models to be used but it is believed that due to the high velocity slot flow it is a good assumption to assume fully turbulent flow. The turbulent kinetic energy $k$ and is dissipation rate $\epsilon$ are given from the following transport equations: ${ }^{2}$

$$
\frac{\partial}{\partial t}(\rho k)+\frac{\partial}{\partial x_{i}}\left(\rho k u_{i}\right)=\frac{\partial}{\partial x_{j}}\left[\left(\mu+\frac{\mu_{t}}{\sigma_{k}}\right) \frac{\partial k}{\partial x_{j}}\right]+G_{k}+G_{b}-\rho \epsilon-Y_{M}+S_{k}
$$

and

$$
\frac{\partial}{\partial t}(\rho \epsilon)+\frac{\partial}{\partial x_{i}}\left(\rho \epsilon u_{i}\right)=\frac{\partial}{\partial x_{j}}\left[\left(\mu+\frac{\mu_{t}}{\sigma_{\epsilon}}\right) \frac{\partial \epsilon}{\partial x_{j}}\right]+C_{1 \epsilon} \frac{\epsilon}{k}\left(G_{k}+C_{3 \epsilon} G_{b}\right)-C_{2 \epsilon} \rho \frac{\epsilon^{2}}{k}+S_{e}
$$

where $G_{k}$ is the generation of turbulent kinetic energy. This is calculated in a manner described by the Boussinesq approximation. $G_{b}$ is the turbulent kinetic energy added due to buoyancy. $C_{1 \epsilon}, C_{2 \epsilon}$, and $C_{3 \epsilon}$ are constants that are tuned by experimental data. ${ }^{1} \sigma_{k}$ and $\sigma_{\epsilon}$ are the turbulent Prandle numbers for $k$ and $\epsilon$ respectively. If extra source terms are added by the user they are accounted for using $S_{k}$ and $S_{\epsilon}$.

The turbulent viscosity $\mu_{t}$ is calculated using

$$
\mu_{t}=\rho C_{\mu} \frac{k^{2}}{\epsilon}
$$


where $C_{\mu}$ is a constant. The constants as described by White are presented in Table 4.2.1. ${ }^{1}$

\begin{tabular}{|c|c|}
\hline Constant & Value \\
\hline$C_{1 \epsilon}$ & 1.44 \\
$C_{2 \epsilon}$ & 1.92 \\
$C_{\mu}$ & 0.09 \\
$\sigma_{k}$ & 1.0 \\
$\sigma_{\epsilon}$ & 1.3 \\
\hline
\end{tabular}

Table 4.2.1: Constants for the standard $k-\epsilon$ model as described by White. ${ }^{1}$

These constants are not universal for all problems or variations of the $k-\epsilon$ turbulence model and the constants can be tuned for a specific problems.

\subsubsection{Realizable $k-\epsilon$ Model}

The realizable $k-\epsilon$ model is modified in two ways. First, turbulent viscosity is calculated differently from the standard version. Secondly, there is a new transport equation for dissipation rate $\epsilon$. The most applicable benefit for the realizable $k-\epsilon$ model is that it predicts spreading rate better for jets. Considering the wing has both leading and training edge planer jets realizable $k-\epsilon$ is considered the better $k-\epsilon$ model for the application in this paper.

While the transport equation for kinetic energy is identical to the standard version the new turbulent dissipation transport equations is described by

$$
\frac{\partial}{\partial t}(\rho \epsilon)+\frac{\partial}{\partial x_{i}}\left(\rho \epsilon u_{i}\right)=\frac{\partial}{\partial x_{j}}\left[\left(\mu+\frac{\mu_{t}}{\sigma_{\epsilon}}\right) \frac{\partial \epsilon}{\partial x_{j}}\right]+\rho C_{1} S_{\epsilon}-\rho C_{2} \frac{\epsilon^{2}}{k+\sqrt{\nu \epsilon}}+C_{1 \epsilon} \frac{\epsilon}{k} C_{3 \epsilon} G_{b}+S_{\epsilon}
$$


where

$$
\begin{gathered}
C_{1}=\max \left[0.43, \frac{\eta}{\eta+5}\right] \\
\eta=S \frac{k}{\epsilon} \\
S=\sqrt{2 S_{i j} S_{i j}}
\end{gathered}
$$

In the above equations $G_{k}$ is the generation of turbulent kinetic energy due to the mean velocity gradients. As in the standard version the eddy viscosity is calculated using

$$
\mu_{t}=\rho C_{\mu} \frac{k^{2}}{\epsilon}
$$

the difference in the realizable model is the method with which $C_{\mu}$ is calculated. Before $C_{\mu}$ is a constant and now is calculated using

$$
C_{\mu}=\frac{1}{A_{0}+A_{s} \frac{k U^{*}}{\epsilon}}
$$

while I will spare you the details of the rest of the calculation it can be found in full in the FLUENT User Manual. ${ }^{2}$ The constants for this model are listed in Table 4.2.2

\begin{tabular}{|c|c|}
\hline Constant & Value \\
\hline$C_{1 \epsilon}$ & 1.44 \\
$C_{2}$ & 1.9 \\
$\sigma_{k}$ & 1.0 \\
$\sigma_{\epsilon}$ & 1.2 \\
\hline
\end{tabular}

Table 4.2.2: Constants for the realizable $k-\epsilon$ model as described by FLUENT. ${ }^{2}$ 
This version of $k-\epsilon$ shows good results for free shear flows, jet flows and flows with strong mixing layers among many other types. The model tends to fall apart in wall bounded flows with high pressure gradients and flows with high adverse pressure gradients. ${ }^{37}$ It is worthy to note that the realizable $k-\epsilon$ is considered to be superior in accuracy and stability over the standard version of $k-\epsilon$. This is why realizable $k-\epsilon$ is strongly considered as the turbulence model for this project.

\subsubsection{Standard $k-\omega$ Model}

The $k-\omega$ model was developed by Wilcox. ${ }^{3}$ His model improved on some of the shortfalls of other turbulence models SUCH AS: low-Reynolds number effects, compressibility, and shear flow spreading. The model greatly improved prediction of free shear flow spreading for wakes, free shear flows and jets. This makes this turbulence mode attractive because the circulation control jet is a wall bounded planer jet with high free shear flow. The transport equations describing the $k-\omega$ model are as follows:

$$
\frac{\partial}{\partial t}(\rho k)+\frac{\partial}{\partial x_{i}}\left(\rho k u_{i}\right)=\frac{\partial}{\partial x_{j}}\left(\Gamma_{k} \frac{\partial k}{\partial c_{j}}\right)+G_{k}-Y_{k}+S_{k}
$$

and

$$
\frac{\partial}{\partial t}(\rho \omega)+\frac{\partial}{\partial x_{i}}\left(\rho \omega u_{i}\right)=\frac{\partial}{\partial x_{j}}\left(\Gamma_{\omega} \frac{\partial \omega}{\partial x_{j}}\right)+G_{\omega}-Y_{\omega}+S_{\omega}
$$

where $G_{k}$ is the turbulent kinetic energy due to mean velocity gradients and $G_{\omega}$ 
represents the generation of the dissipation rate. $Y_{k}$ and $Y_{\omega}$ are the dissipation of $k$ and $\omega$ due to turbulence. $\Gamma_{k}$ and $\Gamma_{\omega}$ are the effective diffusivity of $k$ and $\omega$ respectively. The effective diffusivity are calculated using

$$
\begin{gathered}
\Gamma_{k}=\mu+\frac{\mu_{t}}{\sigma_{k}} \\
\Gamma_{\omega}=\mu+\frac{\mu_{t}}{\sigma_{\omega}}
\end{gathered}
$$

where $\sigma_{k}$ and $\sigma_{\omega}$ are the turbulent Prandtl numbers for $k$ and $\omega$ respectively. The turbulent viscosity $\mu_{t}$ is calculated by

$$
\mu_{t}=\alpha^{*} \frac{\rho k}{\omega}
$$

where $\alpha *$ is a damping term for the turbulent viscosity correcting for low Reynolds number flows. The term $G_{k}$ (production of turbulence kinetic energy) from above is calculated in the same manner as $k-\epsilon$ using the Boussinesq approximation. The production of $\omega$ given by $G_{\omega}$ is calculated using

$$
G_{\omega}=\alpha \frac{\omega}{k} G_{k}
$$

Some of the model constants given by Wilcox are shown in Table 4.2.3.

While the standard $k-\omega$ model made good progress at improving some of the shortfalls of earlier turbulence models it doesn't come without faults. The standard 


\begin{tabular}{|c|c|}
\hline Constant & Value \\
\hline$\sigma_{k}$ & 2.0 \\
$\sigma_{\omega}$ & 2.0 \\
\hline
\end{tabular}

Table 4.2.3: Constants for the standard $k-\omega$ model as described by Wilcox. ${ }^{3}$

$k-\omega$ model can have issues with solution instability and divergence in freestream flow outside of boundary layer. For this reason a new formulation of the $k-\omega$ model was developed called the shear stress transport $k-\omega$ model.

\subsubsection{Shear Stress Transport $k-\omega$ Model}

The Shear Stress Transport $k-\omega$ model was developed by Menter in the early 90 's. ${ }^{38}$ Menter combined the near wall accuracy of the $k-\omega$ model and utilizes the $k-\epsilon$ model in freestream flow away from walls. This coupling provides a balance between accuracy and stability that creates a powerful turbulence model. The blended model performs exceptionally well in adverse pressure gradient flows. This makes it very attractive for circulation control airfoil flow. The transport equations for $k-\omega$ SST are a slight variant on the standard $k-\omega$ model. They are written as follows

$$
\frac{\partial}{\partial t}(\rho k)+\frac{\partial}{\partial x_{i}}\left(\rho k u_{i}\right)=\frac{\partial}{\partial x_{j}}\left(\Gamma_{k} \frac{\partial k}{\partial c_{j}}\right)+\tilde{G}_{k}-Y_{k}+S_{k}
$$

and

$$
\frac{\partial}{\partial t}(\rho \omega)+\frac{\partial}{\partial x_{i}}\left(\rho \omega u_{i}\right)=\frac{\partial}{\partial x_{j}}\left(\Gamma_{\omega} \frac{\partial \omega}{\partial x_{j}}\right)+G_{\omega}-Y_{\omega}+D_{\omega}+S_{\omega}
$$

where notable differences are $\tilde{G}_{k}$ represents the generation of turbulent kinetic energy 
due to mean velocity gradients. $\tilde{G}_{k}$ is calculated as follows:

$$
\tilde{G}_{k}=\min \left(G_{k}, 10 \beta^{*} k \omega\right)
$$

$G_{k}$ is calculated the exact same way as in the standard $k-\omega$ model shown in Equation 4.2.18. $D_{\omega}$ is the cross-diffusion term and is calculated as described below. All other terms are consistent with the standard $k-\omega$ model. In order to blend the $k-\omega$ and the $k-\epsilon$ models together the cross-diffusive term $D_{\omega}$ is added to Equation 4.2.20. $D_{\omega}$ is calculated as follows

$$
D_{\omega}=2\left(1-F_{1}\right) \rho \sigma_{\omega, 2} \frac{1}{\omega} \frac{\partial k}{\partial x_{j}} \frac{\partial \omega}{\partial x_{j}}
$$

to see the rest of the derivation please see Menter or FLUENT User Manual. ${ }^{38}{ }^{2}$ Some of the constants are given in Table 4.2.4

\begin{tabular}{|c|c|}
\hline Constant & Value \\
\hline$\sigma_{k, 1}$ & 1.176 \\
$\sigma_{k, 2}$ & 1.0 \\
$\sigma_{\omega, 1}$ & 2.0 \\
$\sigma_{\omega, 2}$ & 1.168 \\
$\beta^{*}$ & 0.09 \\
\hline
\end{tabular}

Table 4.2.4: Constants for the shear stress transport $k-\omega$ model as described by FLUENT. ${ }^{2}$

\subsubsection{Wall Treatment}

Near wall treatment in turbulence models can significantly effect the accuracy of the solution. Walls are the main contributor to mean velocity and turbulence within a solution. There are large gradients in velocity and pressure near walls. There are 
a number of ways to treat the wall boundary in FLUENT. $k-\epsilon$ model is most valid somewhat far from the wall region. While this is less of a exact distance a nondimensional number $y^{+}$will be presented shortly to give meaning to the statement. $k-\omega$ on the other had is valid all the way to the wall given there is enough resolution. $y^{+}$will also give meaning to the previous statement.

Wall $y^{+}$can be define as

$$
Y^{+}=\frac{\rho \mu_{\tau} y}{\mu}
$$

where $y$ is the distance from the wall and $\mu_{\tau}$ is the friction velocity defined by

$$
\mu_{\tau}=\sqrt{\frac{\tau_{\omega}}{\rho}}
$$

For turbulence model evaluation it is convenient to divide the near wall region into three layers. Closest to the wall is the viscous sub layer, above that the buffer layer, and the third layer being the log law region where flow if fully turbulent. A plot of these layers versus $y^{+}$value is plotted in Figure 4.2.1.

The two approaches commonly used to evaluate this near wall region is wall functions and modeling all the way to very close to the wall. The wall function approach uses semi-empirical formulas to approximate the boundary layer from the log-law region down to the wall face. This approach requires the first cell height to range between a $y^{+}$value of $60-300$. This shows the best correlation with experimental data for various flow regimes. 


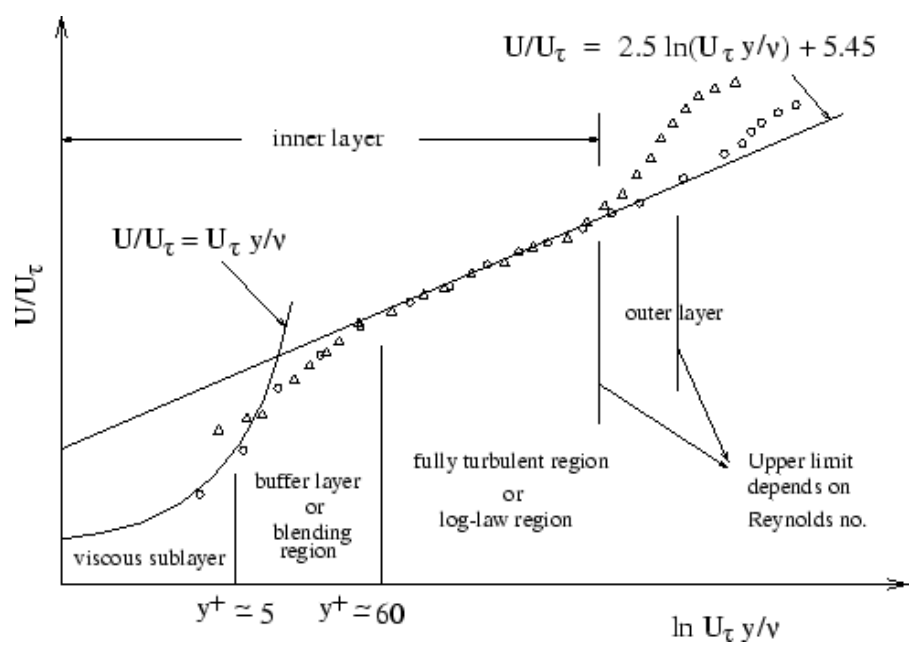

Figure 4.2.1: Non-Dimensionalized velocity plotted versus $y^{+}$shoing subdivisions of nera wall region. ${ }^{2}$

The near wall method, modeling all the way down to the wall, requires a much smaller $y^{+}$value of less than five. Ideally $y^{+}$should be approximately one everywhere. The is no lower limit on this value because as the $y^{+}$approaches zero the more accurate the model becomes. In applied CFD it is generally accepted that a $y^{+}$of approximately one is a good first layer height. Figure 4.2.2 illustrates the difference in mesh refinement between the two methods.

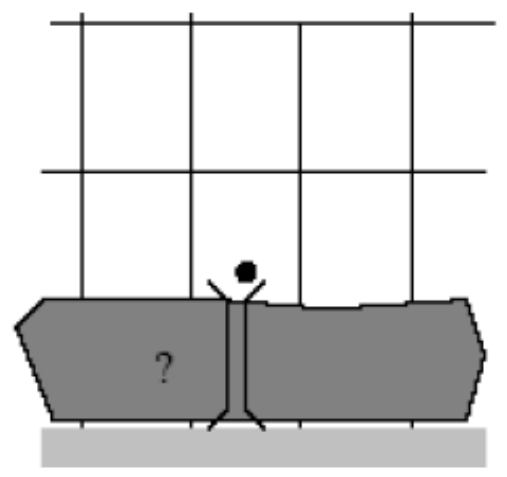

Wall Function Approach

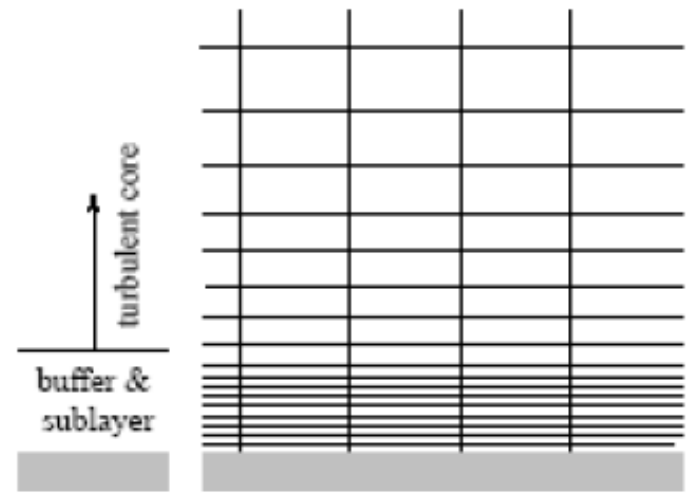

Near-Wall Model Approach

Figure 4.2.2: Wall functions versus near wall approach. ${ }^{2}$ 
In FLUENT the $k-\epsilon$ turbulence model allows you to specify either wall functions or enhanced wall treatment. Wall functions utilize the empirical formulas discussed above for all near wall cells. Enhanced wall treatment allows for wall functions on cells with $y^{+}$values above 5 and for near wall treatment for $y^{+}$values below 5 . This is a convenient way to allow for a turbulence model to utilize both approaches with one method.

Wall functions upside is that meshes are much easier to create since many less prism cells need to be clustered near the surface. This lowers cell count thus reducing solving time. Wall functions perform well in steady non-separated flow. The downside of wall functions is that they begin to fall apart when they are used outside of the idealized flow conditions they were designed perform under. Some flow regimes where wall functions tend to break down are as follows: low Reynolds number flow, flow through small channels, high pressure gradients leading to separation, and strong 3D boundary layers.

In FLUENT $k-\omega$ treats all cells as near wall cells whether they are near wall cells or not. This means that the mesh provided needs to endure that $y^{+}$values is below five so that the wall will be treated correctly. The near wall approach works well in many flow regimes because doesn't make guesses to the flow in the log-law and viscous sub layer because it resolves these areas. The downside of course is that the meshes are much harder to make and cell count can increase rapidly. 


\subsection{Blowing Coefficient}

3D blowing Coefficient $\left(C_{\mu}\right)$, also noted as momentum coefficient in some literature, is a non-dimensional number measuring the 'strength" of the circulation control slot. The strength has a theoretical maximum based on the geometry of the slot (slot exit area) and the freestream conditions. The slot geometry was set based on the wind tunnel model so it in order to achieve a target $C_{\mu}$ of 0.5 the freestream conditions need to be set such that the target can be achieved. Having too high of a freestream and the desired blowing coefficient may not be attainable. $C_{\mu}$ can be calculated with the following ${ }^{8}$

$$
C_{\mu}=\frac{\dot{m} U_{j e t}}{q_{\infty} S}
$$

where $U_{j e t}$ is the velocity at the slot and $S$ is the reference area. The $C_{\mu}$ is then limited by the maximum velocity achievable at the slot. For all purposes the slot is not a "designed" nozzle so its reasonable to suspect that the fastest acheiveable velocity lies somewhere slightly below Mach 1 . With three variables set $U_{j e t}, \dot{m}$ and $S$, the remaining sole remaining variable dynamic pressure $\left(q_{\infty}\right)$ becomes a sliding scale of which you can set the theoretical maximum $C_{\mu}$. Solving for $q_{\infty}$ and setting a maximum $C_{\mu}$ target a freestream dynamic pressure for a desired $C_{\mu}$ can be found Equation 4.3.2. 


$$
q_{\infty}=\frac{\dot{m} U_{j e t}}{C_{\mu} S}
$$

Targeting a maximum $C_{\mu}$ of approximately 0.6 a freesream dynamic pressure is backed out of around $350 \mathrm{~kg} / \mathrm{ms}^{2}$. At sea level standard day the dynamic pressure equates to a freestream Mach number of 0.07 . This will serve as the Mach for all test runs and the slot conditions will be set such that a target $C_{\mu}$ of 0.5 can be achieved.

Measuring the $C_{\mu}$ on the CFD models to verify the correct boundary conditions were set is a little tricky. Allen discusses the difficulty of accurately measuring the $C_{\mu}$ and gives recommendations on how to do it. ${ }^{39}$ Measuring $C_{\mu}$ on a slot that moves in $3 \mathrm{D}$ and twists is not as easy as taking a surface integral at the slot exit. The geometry is such that a surface is not able to be built. The solution is the mass flow rate is known because it is set at the plenum inlet. The velocity is measured at the slot outlet by taking a very large number of point velocities and then averaging out

to get a average slot velocity. This method was also used by Shovin et. al. ${ }^{21}$ This was validated on a simple CCW CFD solution and the results showed an excellent correlation. Then using the freestream conditions $C_{\mu}$ can be calculated.

\subsection{CFD Validation - Turbulence Model Evalua- tion}

Historically turbulence models have been poor in predicting the lift and drag on circulation control wings. This can partly be attributed to the fact that traditional 
RANS turbulence models have a hard time predicting the shear layer between the high momentum jet and the lower momentum freestream flow. For circulation control airfoils the increase in lift is mostly caused by the moving of the separation point, this is very hard to predict in the high momentum slot flow. While an effort is being made at Cal Poly to develop turbulence models that more accurately predict $\mathrm{CCW}$ flow they were not available for this project..$^{9,40}$

A number of studies have been performed as part of Cal Poly's Hybrid Wing Body NASA research grant. Two in particular will be discussed in detail and will serve as a basis for turbulence model selection. The first experiment was part of an effort to develop the $v^{2}-f$ turbulence model in the process a number of other turbulence models were explored. This work was performed by Storm. ${ }^{40}$

\subsubsection{D Circulation Control Airfoil}

Storm utilized some experimental data on a General Aviation Circulation Control (GACC) airfoil to compare his CFD to. The airfoil can be seen in Figure 4.4.1. The airfoil geometry has a slot over the upper flap surface. The geometry departs from a traditional rounded trailing edge and incorporates a dual radius flap similar to the geometry in this paper. The experimental work was performed by Lee-Rausch et al and Jones et al. ${ }^{41,8}$

Comparison cases were run over a range of blowing coefficients, $C_{\mu}$, to compare against the experimental data. The original CFD data from Storm included the one equation Spalart-Allmaras data but it was removed because it performed poorly and 


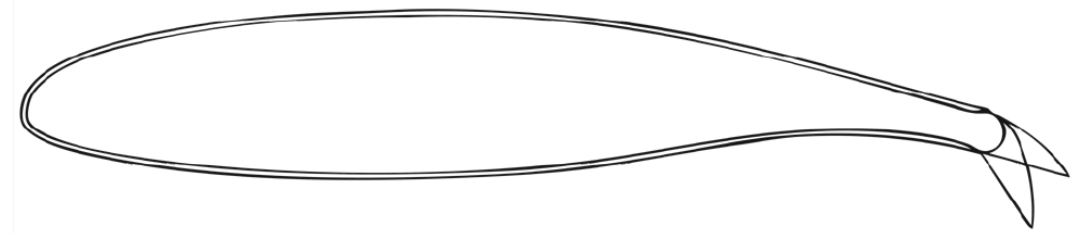

Figure 4.4.1: GACC validation airfoil

was never discussed as a possible turbulence model for this work. Results shown in Figure 4.4.2 are all on structure meshes with $y^{+}$of less than 1. Lift coefficient was monitored to determine convergence.

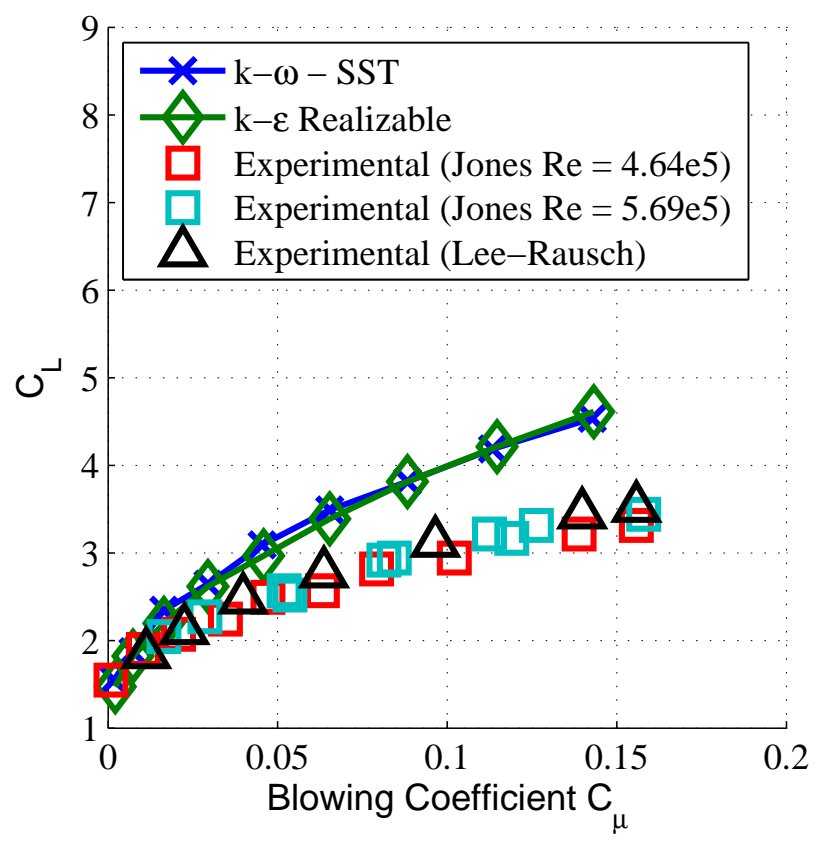

Figure 4.4.2: Blowing coefficient versus lift coefficient of a circulation control airfoil from Storm. ${ }^{8,9}$

It is worth noting that the momentum coefficients shown in this study are much lower than the coefficients studied on the geometry for this paper. Even though the $k-\omega$ and the $k-\epsilon$ turbulence models showed very similar results in the validation shown above the $k-\omega$ model was selected because it is generally considered more 
accurate. This model is particularly attractive because of its performance in high adverse pressure regions. It is also very good with planer jets. All CFD work observed shows clear over-prediction of lift forces and as the momentum coefficient increases as does over-prediction. The above work clearly shows that in order to predict actual $C_{L}$ values (not just trends) a new turbulence model will be required. For the purposes of this work trends are considered accurate enough that a new solving technique is not required.

\subsubsection{D Circulation Control Wing Section with Engine Sim- ulator}

To ensure that this $2 \mathrm{D}$ data translates to a full 3D model another validation effort was made into a wind tunnel experiment by Georgia Tech Research Institute (GTRI). ${ }^{42}$ This will serve as a closer resemblance of the $2 \mathrm{D}$ study because the meshing and solving practices will be the same as those for the geometry for this paper. The validation work was performed by Marcos and multiple cases were run by this author to confirm select points to ensure quality and consistency between the work. Since all the test points were confirmed between the two authors the full work by Marcos will be presented here. ${ }^{43}$

The GTRI geometry consists of a circulation control wing with a trailing edge slot over a dual radius flap geometry. There is an engine simulator mounted over the wing. The geometry is shown in Figure 4.4.3. The mesh for this geometry was 8 million cells and meshed in the same hybrid manner as previously discussed with a 
unstructured mesh close to the geometry with structured hexahedrals in the far field domain. The $y^{+}$was targeted to be one and $95 \%$ of the cells being below two.

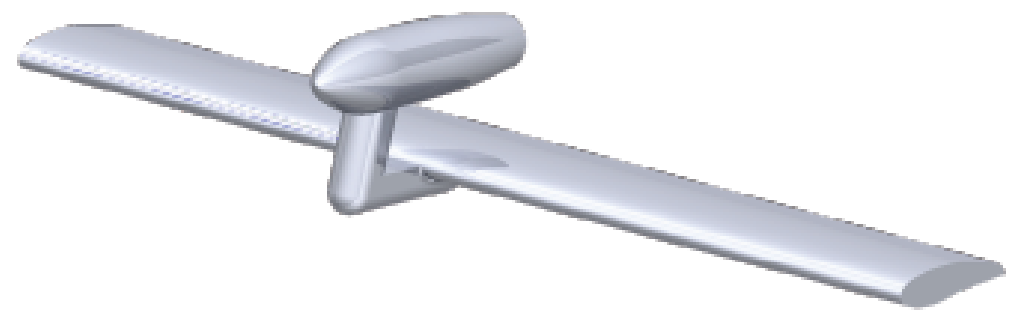

Figure 4.4.3: GTRI wind tunnel geometry for validation cases. ${ }^{10}$

The GTRI model was run at a wide range of thrust and moment coefficients. Some of the results from Marcos and Englar are shown in Figure 4.4.4. ${ }^{10,42}$

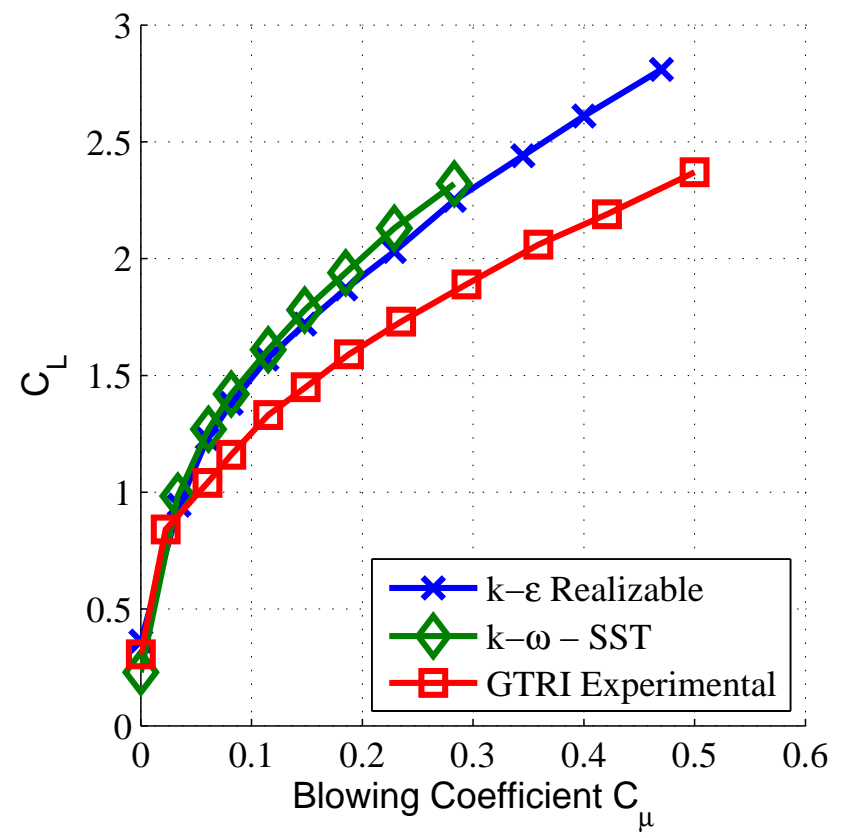

Figure 4.4.4: Blowing coefficient versus lift coefficient of a circulation control airfoil from

As can be seen above both turbulence models consistently over predict lift. As was the case with the 2D GACC airfoil the higher the momentum coefficient the more 
the model over predicts.

Considering that both the turbulence models are very closely aligned with each other for both studies it was necessary to make a decision based on knowledge of the inner workings of turbulence models. $k-\omega$ SST was selected because of its ability to better predict flow with high adverse pressure regions and wall bounded flows. The circulation control jets were of particular importance and $k-\omega$ is known to perform better with planer jets.

\subsection{Computational Resources}

As noted previously one of the more challenging parts to this work was the computational resources limiting the size and number of the solutions produced. The solutions were solved on a 92 core cluster with 184 gigabytes of RAM. The RAM limited the cluster to solving a maximum of approximately 40 million cell meshes. The meshes for this work were solved on this cluster in approximately 7-10 days. For pre and post processing an 8 core 64 gigabyte RAM desktop machine was used. 


\section{Chapter 5}

\section{Results}

\subsection{Preliminary Results}

A proof of concept geometry was mocked up to evaluate the effectiveness of this geometry to entrain engine exhaust. The geometry for this preliminary study was slightly different than the work in the remainder of this paper. The wing planform stayed constant but different airfoils and twists were used in this version. The main difference was that the pylon was modeled and the engine was a generic nacelle with mixed exhaust. The geometry can be seen in Figure 5.1.1.

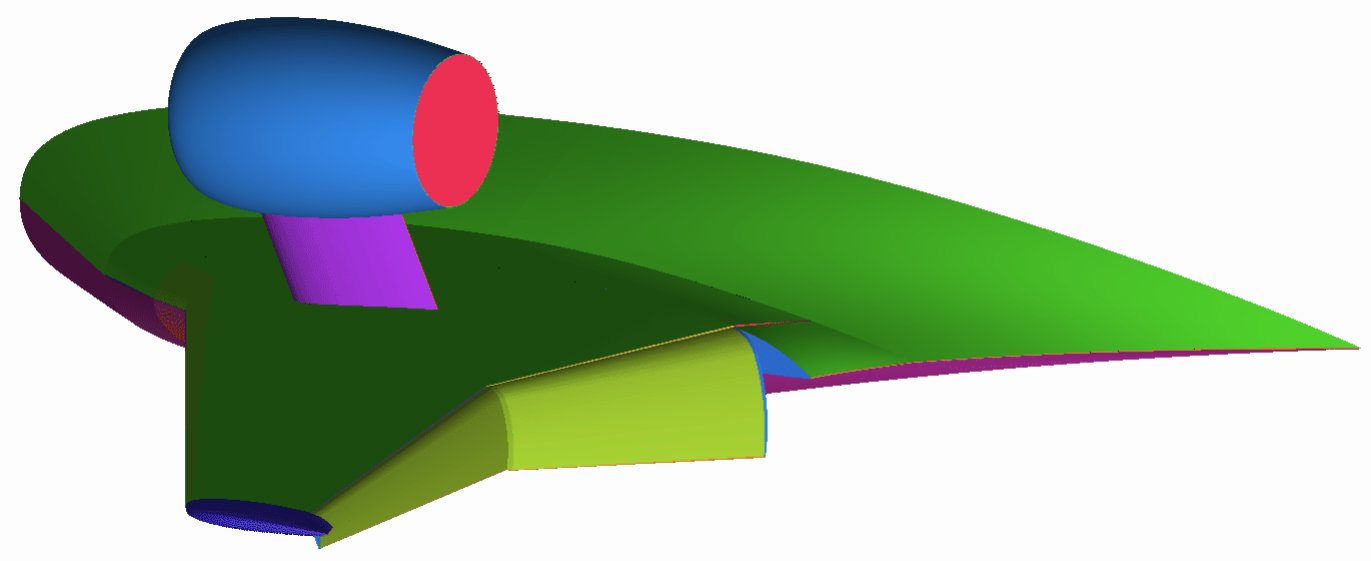

Figure 5.1.1: Preliminary geometry for proof of concept study. 
This study included four engine heights and the pylon was extruded to keep the same basic shape. The engine exit plane maintained the same $58 \%$ chord location. Boundary conditions for this geometry were quickly set up before the thrust coefficients of the engine and momentum coefficient of the slot were known. Relevant boundary conditions are summarized in Table 5.1.1.

\begin{tabular}{|c|c|}
\hline Boundary Condition & Value \\
\hline$C_{\mu}$ & 0.6 \\
$C_{T}$ & 0.75 \\
Mach & 0.1 \\
$P_{\infty}$ & $14.7 \mathrm{Psi}$ \\
$T_{\infty}$ & $518.7 \mathrm{R}$ \\
$\rho_{\infty}$ & 0.00238 slugs $/ \mathrm{ft}^{3}$ \\
\hline
\end{tabular}

Table 5.1.1: Summary of boundary conditions for proof of concept solutions.

These boundary conditions do not reflect the boundary conditions used for the remainder of this project. The results showed that all of the engine flow was significantly entrained. Figure 5.1.2 shows Mach contours of two different engine heights. It is clear that there is entrainment and this served as a proof of concept so a better experiment could be formed to analyze the design space and compare against previous wind tunnel results.

\subsection{Test Matrix}

The test points are taken from work previously performed by Englar. ${ }^{7}$ In an attempt to obtain high engine deflection angles, a high blowing coefficient, $C_{\mu}$, is targeted. For all cases the target $C_{\mu}$ is 0.5 . The thrust coefficient is defined by the 


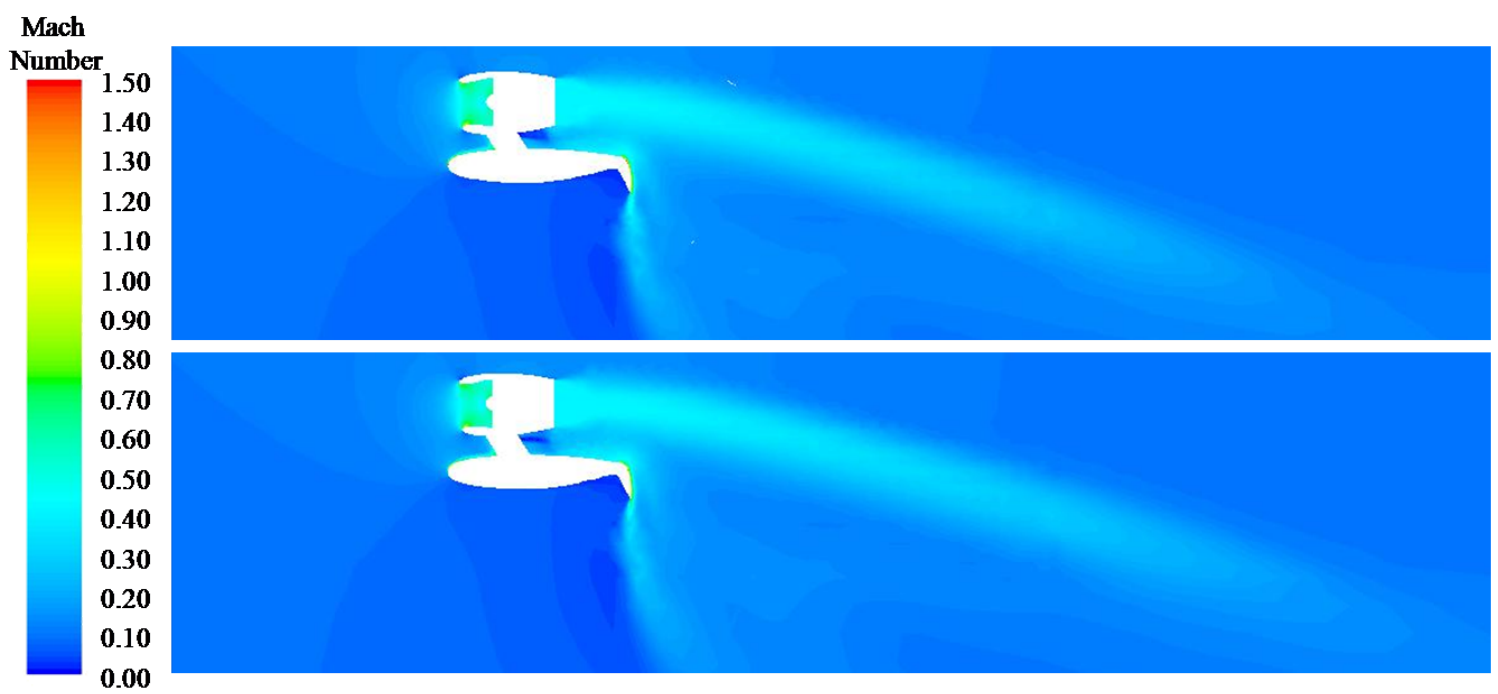

Figure 5.1.2: Mach contours of early entrainment results shown as a proof of concept.

capabilities of the turbofan propulsion simulator (TPS) capabilities. Since the TPS engine geometry is designed for certain capabilities in the wind tunnel it was advantageous to use thrust capabilities that are realistic. The thrust coefficient for some of the wind tunnel test runs targets approximately 0.85 and the same coefficient will be used in all of the CFD presented in the rest of this paper. Table 5.3.1 is a listing of the CFD test cases. The engine locations are designed to cover a spread of locations that are noted by Engler to give a range of lift coefficients (engine entrainment numbers).

Part of being able to characterize engine entrainment based on engine location involves limiting the number of variables in the problem. This is done so the increase or decrease in engine deflection can be directly related to the change in engine position. Some of the variables removed include; engine pylon (Section 2.2), blowing coefficient chosen for high engine deflection (Section 2.1), thrust coefficient based engine simulator capabilities, and freestream conditions (Section 5.3). The locations 


\begin{tabular}{|c|c|c|c|c|}
\hline Case Name & $\begin{array}{c}\text { Engine Chord } \\
\text { Location (\% Chord) }\end{array}$ & $\begin{array}{c}\text { Engine Height } \\
\left(\frac{Z}{D_{\text {eng }}}\right)\end{array}$ & $\begin{array}{c}C_{\mu} \\
\text { Target }\end{array}$ & $\begin{array}{c}C_{T} \\
\text { Target }\end{array}$ \\
\hline Baseline (No Engine) & - & - & 0.50 & - \\
Geometry 1 & 3 & 0.19 & 0.50 & 0.85 \\
Geometry 2 & 3 & 1.02 & 0.50 & 0.85 \\
Geometry 3 & 13 & 0.30 & 0.50 & 0.85 \\
Geometry 4 & 13 & 0.73 & 0.50 & 0.85 \\
Geometry 5 & 1.20 & 0.50 & 0.85 \\
Geometry 6 & 25 & 0.43 & 0.50 & 0.85 \\
Geometry 6 & 25 & 0.43 & 0.33 & 0.85 \\
Geometry 6 & 25 & 1.20 & 0.10 & 0.85 \\
Geometry 7 & 25 & 0.30 & 0.50 & 0.85 \\
Geometry 8 & 50 & 0.73 & 0.50 & 0.85 \\
Geometry 9 & 50 & 0.50 & 0.85 \\
\hline
\end{tabular}

Table 5.2.1: Listing of cases solved.

described in Table 5.3.1 are visualized in Figure 5.2.1. Each dot represents a CFD test location located by the bottom trailing edge (at engine centerline) of the nacelle. Please refer to the engine location schematic in Figure 5.2.1 for details on engine location definitions.

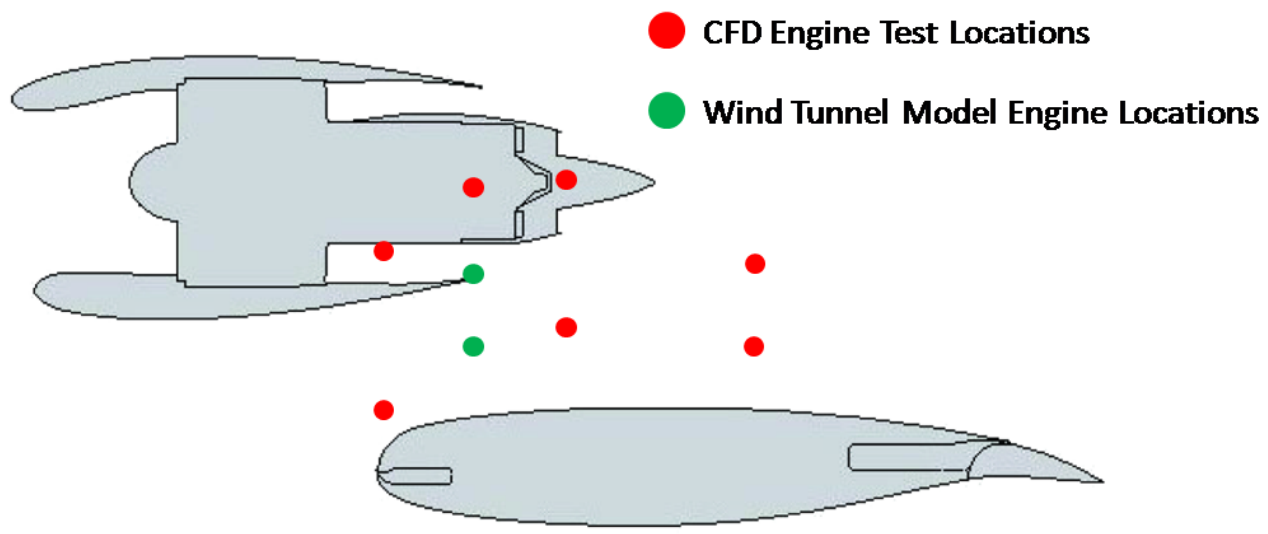

Figure 5.2.1: CFD test locations located by the bottom trailing edge of the nacelle. 


\subsection{Solution Overview}

The solutions presented in this paper are extremely difficult to produce. With so many complex flow regimes and difficult boundary conditions to set, it would be very easy to produce invalid solutions. This was avoided by going through a series of studies and verifications to ensure the highest quality solutions. Some of the solution details and studies are presented in this section. The test conditions are summarized in Table 5.3.1.

\begin{tabular}{|c|c|}
\hline Condition & Value \\
\hline AoA & $0^{\circ}$ \\
Mach & 0.075 \\
Velocity & $50 \mathrm{kts}$ \\
Altitude & $0 \mathrm{ft}$ \\
Pressure & $14.7 \mathrm{psi}$ \\
Density & $0.00238 \frac{\text { slugs }}{f t^{3}}$ \\
Temperature & $518.67 \mathrm{R}$ \\
Flap Deflection & $60^{\circ}$ \\
$C_{T}$ & 0.85 \\
$C_{\mu}$ & $0.50^{*}$ \\
\hline
\end{tabular}

Table 5.3.1: Test conditions held constant for all runs. ${ }^{*}$ Target $C_{\mu}$ changed in some select cases

\subsubsection{Solver}

The solutions are all run under the steady state assumption. Solutions are solved with a second order accurate density based solver with the energy equation turned on. Density is calculated on an iteration basis using ideal gas law. Viscosity is also calculated in the same manner using Sutherland's law. Wall functions are not necessary because the $y^{+}$is targeted to be below one everywhere. 


\subsubsection{Wall $y^{+}$}

As noted in the turbulence modeling section (4.2) the wall $y^{+}$is an important variable to get correct when using a turbulence model. Since the $k-\omega$ SST model is being used as the turbulence model a $y^{+}$of one mesh needs to be targeted. A contour plot of the $y^{+}$values is shown in Figure 5.3.1. Overall the cells are below the limit of five and many are clustered below one.

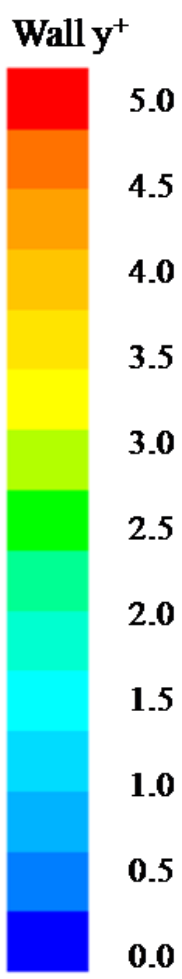

Figure 5.3.1: Contours of wall $y^{+}$.

Some spikes exist in the $y^{+}$data which is to be expected around in the slots and over the flap and wing upper surfaces where the local velocity is greatly accelerated due to high speed slot flow. The contour values are taken and plotted graphically to 
show some of the spikes in $y^{+}$. Figure 5.3.2 plots a selection of values for the specific areas listed in the legend. Considering the wide range of velocities in the solutions the $y^{+}$are nicely grouped below five with many cells being approximately one. As noted in Section 4.2.6 the $y^{+}$could be lowered to group more cells less than one to improve accuracy, but due to mesh size limitations and geometric mesh difficulties the wall $y^{+}$was not modified because it satisfies the turbulence model requirements.

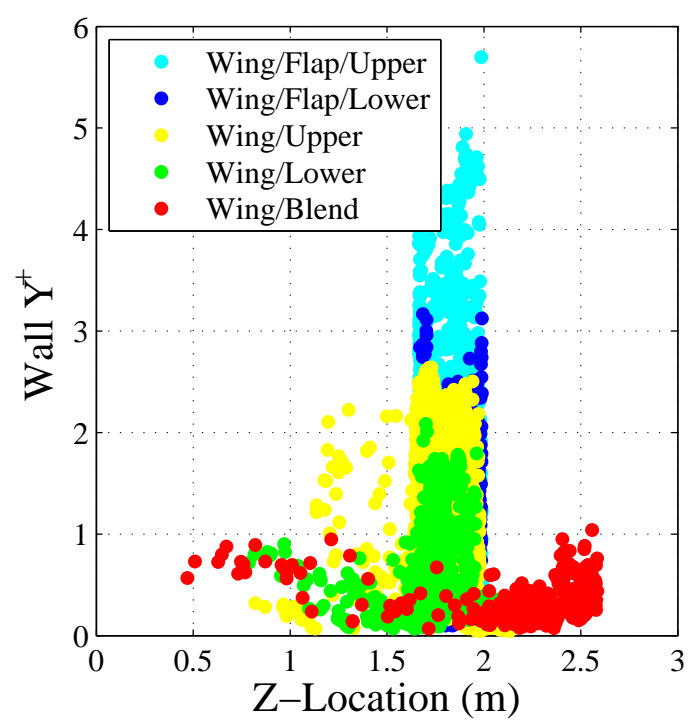

Figure 5.3.2: Graph of $y^{+}$values.

All solutions will maintain the same first cell height so the $y^{+}$values should stay relatively constant between solutions. Not only should consistency be maintained for $y^{+}$but, boundary conditions need to be set and monitored so that they stay consistent as well. 


\subsubsection{Boundary Conditions}

Boundary conditions need to be set at the farfield, plenum inlets, engine fan face, and engine exit for each solution. Each are set to achieve a specific mass flow rate or velocity. The boundary conditions were selected to imitate the conditions that will be seen in the wind tunnel experiment. This was done because the geometry, engine simulator, and circulation control plenums were designed for the wind tunnel test.

\section{Engine Boundary Condition}

The engine has three boundary conditions that need to be set. The fan face is a pressure outlet to the system, the engine bypass exit is a mass flow inlet, and the engine core flow exit is a mass flow inlet. Figure 5.3.3 shows the boundary planes for the engine. In order to maintain an accurate representation of the TPS unit the pressure on the fan face is changed until its mass flow rate matches the mass flow prescribed on the engine bypass exit. This maintains a conservation of mass between the two boundary conditions. The engine nozzle exit is controlled by prescribing a mass flow into the system at the computational plane. This added mass counts as an addition to the overall mass of the system. This is done because the TPS unit works in the same manner during the wind tunnel test. The TPS unit is powered by compressed air being pumped into the wind tunnel, up through the sting, pushed through the core, driving the fan blades, and exiting out the nozzle exit. 


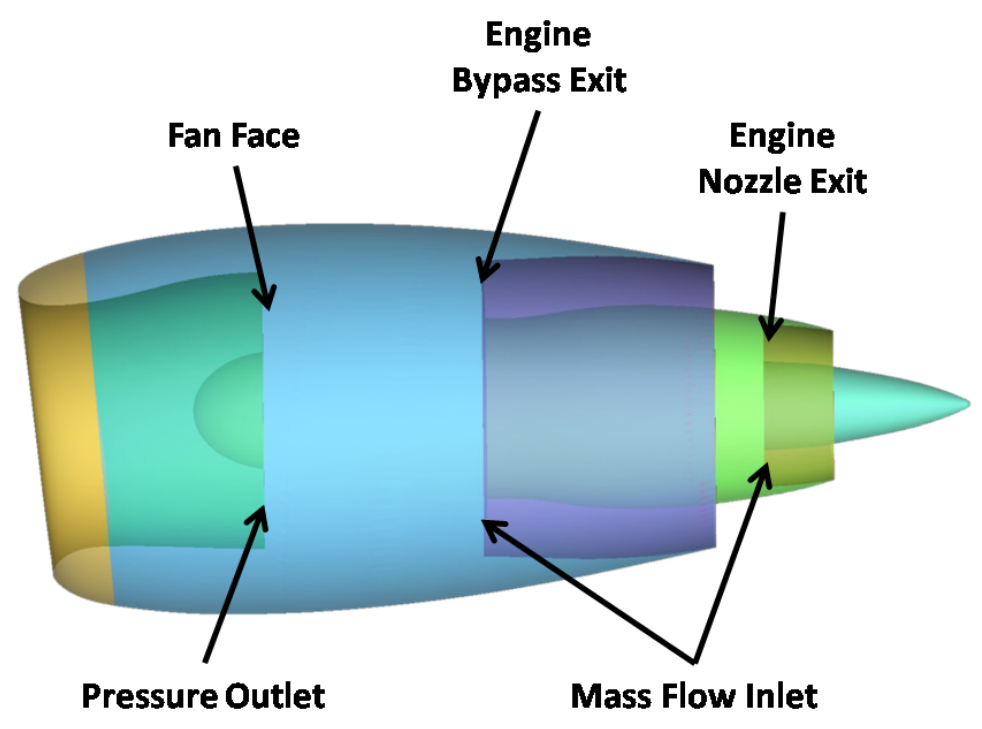

Figure 5.3.3: Engine computational boundary planes and types.

\section{Slot Boundary Condition}

The slot boundary is an interesting and difficult boundary condition to set. There are two approaches to setting the boundary condition on a slot. Baker describes both in detail and the effect on solve time using each approach. ${ }^{44}$ The first approach is to model the computational boundary at the slot exit. The second approach is to model the computational boundary upstream of the slot exit in the plenum.

The advantages of the first method are that it can dramatically lower cell count and thus reduce solve time. This approach can be tricky to implement because knowing the slot conditions in a complex 3D slot is not feasible. It also eliminates the ability to incorporate the velocity profile that would build up in the plenum and throat area. There are some ways to implement this velocity profile in 2D applications using FLUENT's User Defined Functions, but it becomes impossible with a 3D slot 
of this nature.

The advantages of modeling the plenum is that it fixes the boundary layer build up problem that exists in the previous method. The flow is allowed to develop freely as it would in the wind tunnel model. The downside to this approach is that the mesh complexity increases due to the small nature of the slot. See Section 2.3 for complete details. The increase in mesh size for the geometry in this paper is approximately 8 million cells. A schematic showing the two computational boundary locations is shown in Figure 5.3.4.

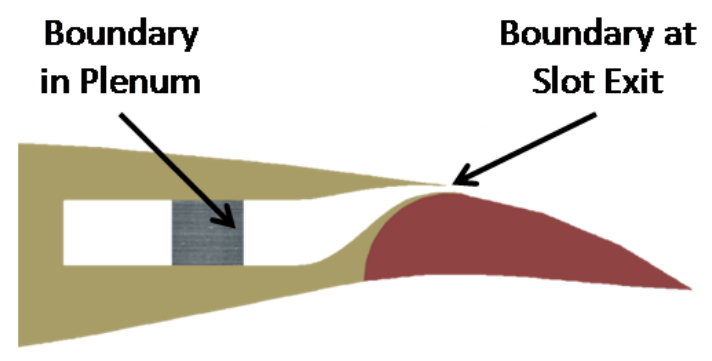

Figure 5.3.4: Computational boundary comparison for slot geometry. Computational plane modeled at slot exit (left) and computational plane modeled in plenum (right).

Both boundary conditions were modeled to compare the slot conditions. The slots were targeted to have a total blowing coefficient of 0.50 and the solution slices compared in Figure 5.3.5 are taken at the same wing section. Modeling the computational boundary at the slot is shown to be a poor choice considering the under expansion of the jet leaving the slot. It is also hard to set a specific $C_{\mu}$ due to the unsteady and divergent nature of the boundary condition. For this approach the mass flow has to be ramped up over many iterations to keep the solution from diverging. The plenum approach paid a cost in time per iteration by adding 8 million cells to the domain, 
but no ramp up was required and the solution was more stable. This second approach also allowed for the boundary layer to build up correctly.
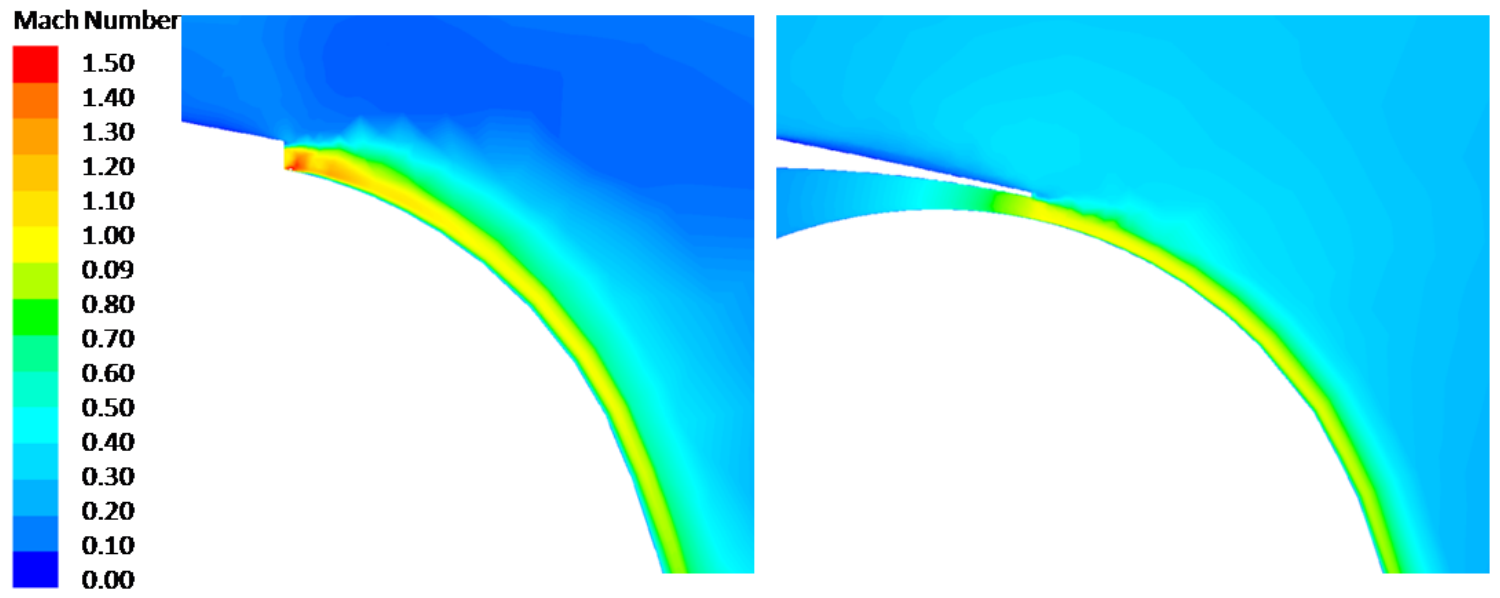

Figure 5.3.5: Mach contours comparing the two slot modeling approaches.

In FLUENT a mass flow inlet is used when the boundary is set at the slot exit. The mass flow is determined based on isentropic hand calculations and it is believed that this boundary condition not only caused the overexpansion, but also the solution instability. For the plenum approach a pressure inlet is used. The boundary condition is determined by the pressure expectations in the tunnel. This is then adjusted slightly in order to get the approximate target $C_{\mu}$. In the end the plenum approach is chosen because it is more accurate and stable than the first approach. The increase in time per iteration is an acceptable drawback.

\section{Farfield Boundary Condition}

The farfield is set to be sufficiently far away that there is no interaction between the domain boundary and the local flow around the model. With this assumption, the 
farfield boundary can be set as a pressure farfield. In FLUENT the pressure farfield boundary condition allows the user to prescribe the Mach number and direction vector for the entire domain. This is used for all solutions.

\subsubsection{Convergence}

Convergence is determined from monitoring the lift on the entire body. Since the solutions contain highly complex flow, including separation and large vortices, the solutions are considered converged when the force monitors showed oscillatory behavior. Figure 5.3.6 shows the oscillatory behavior of one of the solutions. Solutions with behavior such as this are considered to be a converged solution.

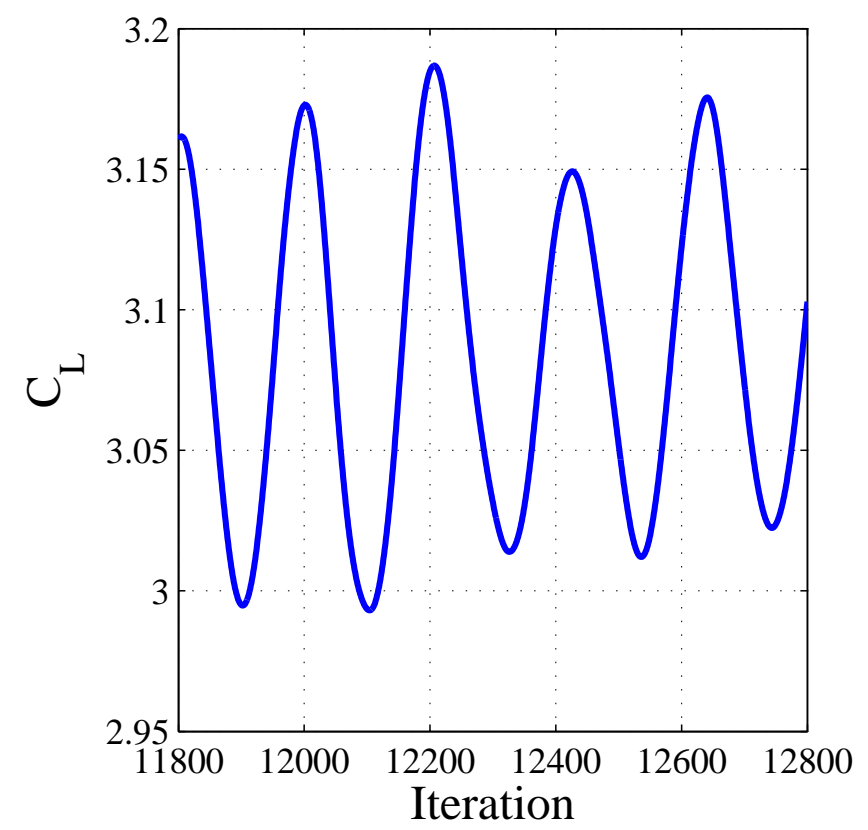

Figure 5.3.6: Oscillatory behavior of a converged solution.

The amplitude of oscillations are often between $5-10 \%$ of the overall lift coefficient. 
This makes it difficult to compare one geometry to the next using lift coefficient because the performance increase/decrease from changing engine location is washed out by these oscillations. The wing is dominating the overall lift coefficient and the increase in $C_{\mu}$ due to engine entrainment is getting lost in the noise. The wing's complex flow also contributes to the unsteady behavior of the solution.

\subsubsection{General Solution Characteristics}

The solutions for this paper contain incredibly complex flow stemming from the circulation control slots changing the "traditional" flow paths over the wing. Due to the high momentum air in the slot blowing perpendicular to the wing leading edge traditional flow paths across a wing are changed. Figure 5.3.7 demonstrates the high cross flow over the upper surface of the wing. The effect is particularly complex where a kink exists in the wing.

The slots interacting with the freestream cause large vortices to develop around the wing. In particular, the end of the flaps cause huge vortices to develop when the circulation control slots are active. These vortices would not only pose problems for airport operation, but adds to the overall complexity and unsteadiness of the flow. The flap vortices that exist in typical aircraft are amplified due to the added energy of the slot pouring over the flap side. The flow exiting off the flap can be observed in Figure 5.3.8. Jones et al. also noticed the vortices under blowing operation are much stronger than in no blowing. ${ }^{8}$ 


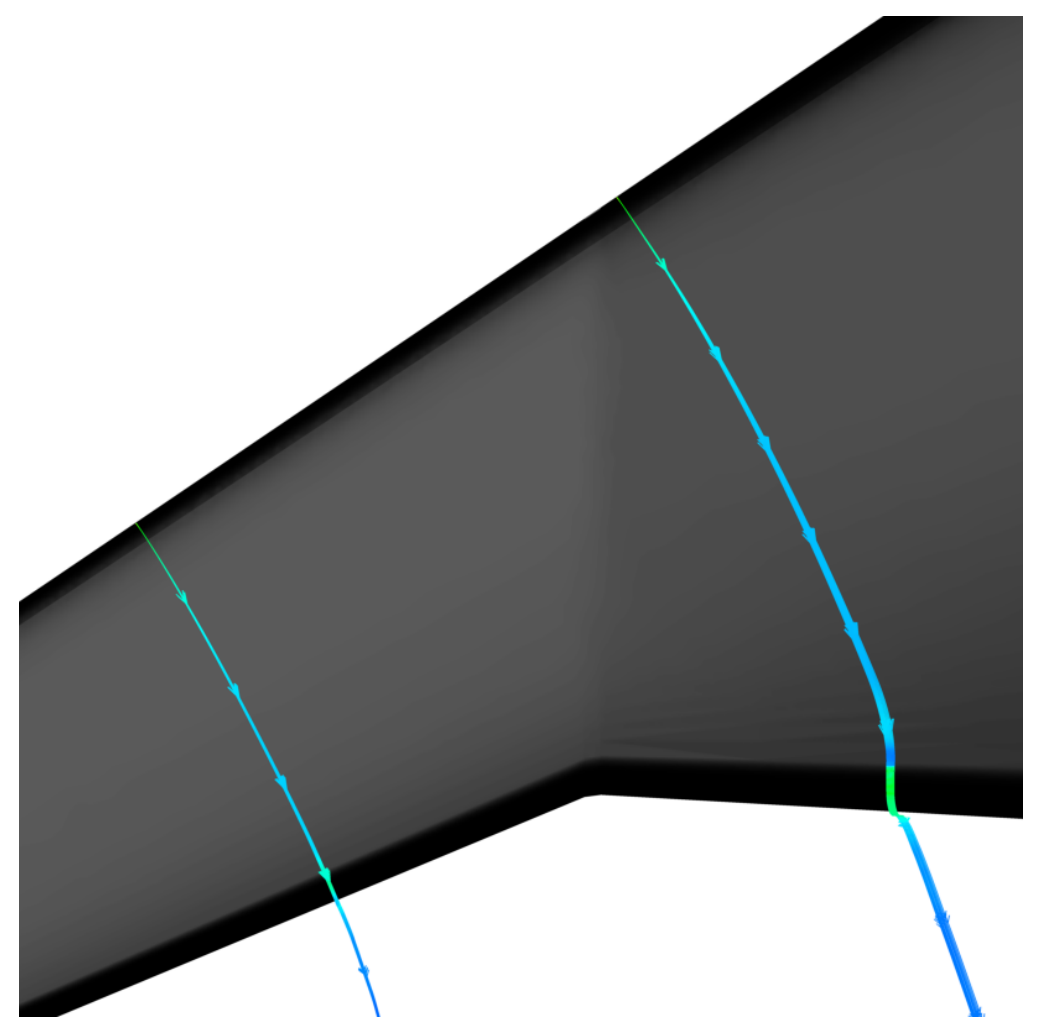

Figure 5.3.7: Streamlines showing flow path over the wing.

\subsection{Evaluating Entrainment Performance}

Due to the unsteadiness of the solution apparent in the oscillations of the force monitors it may be hard to evaluate the performance of a particular setup based on lift coefficient. In the beginning there were two schools of thought to obtaining a parameter to measure the resulting lift that can be generated by a certain configuration. The first variable being conventional lift coefficient and the second being deflection angle of the engine thrust.

Deflection angle of the engine thrust is an attractive parameter because it is the direct reaction to the parameters being observed. The problem being that there is no 


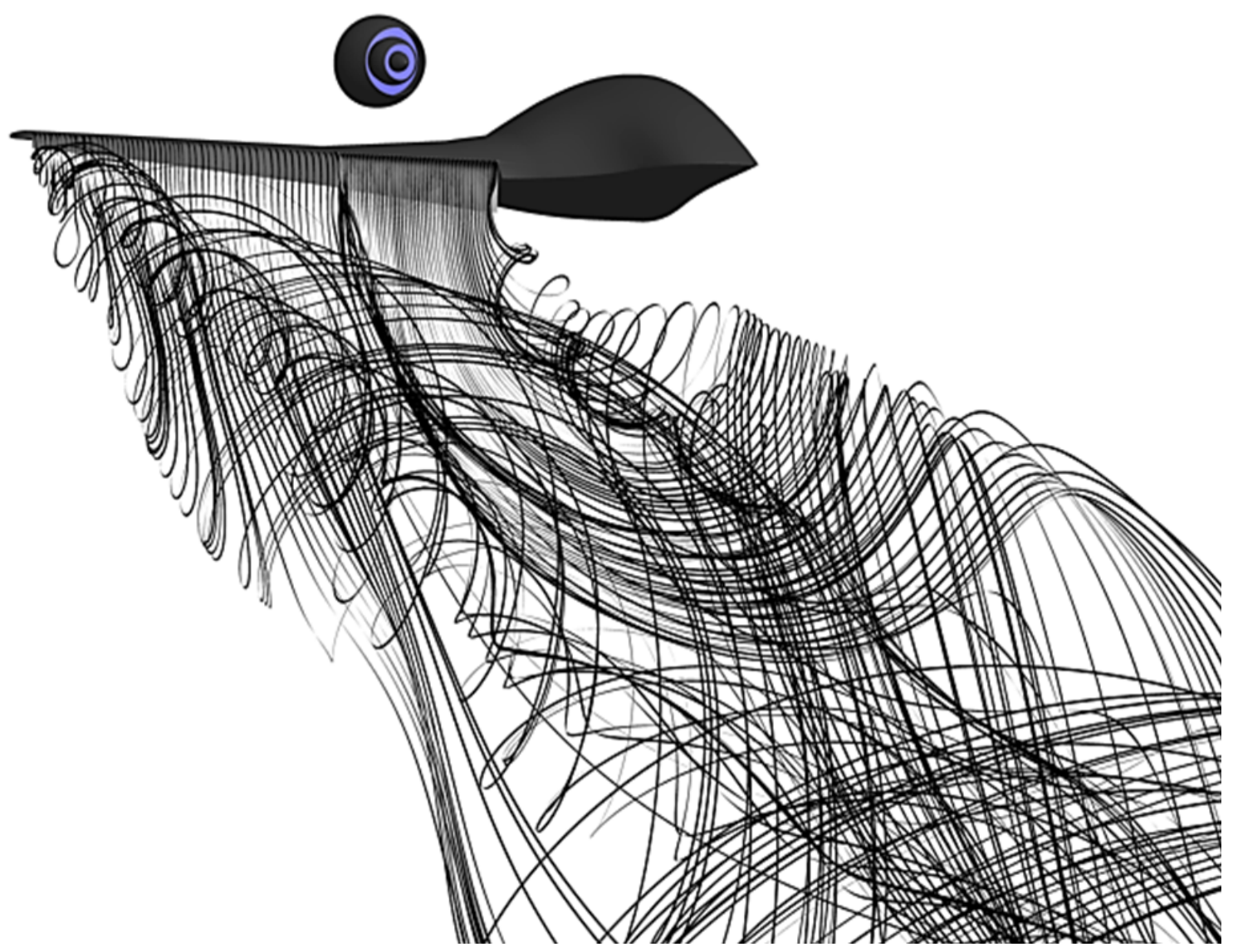

Figure 5.3.8: Streamlines stemming from the flaps showing large vortices.

way to measure engine deflection angle accurately in an experiment. In CFD there are a few ways to calculate this angle, but without validation it may not be the best approach to use.

Lift coefficient is a great value because in the end this is the most applicable unit we can use to assess the real world value of this problem. For example, increasing the lift coefficient under takeoff conditions can directly relate to reducing the takeoff length. Conventional wisdom would suggest that the engine exhaust deflection angle would correlate directly with lift coefficient, but this turned out to not be true. As 
discussed in the previous section, an issue arises when trying to use lift coefficient to evaluate a problem because of the unsteady nature of the solution.

The solutions tended to oscillate in their lift convergence as much as $7 \%$ of the total lift coefficient. Even with averaging $C_{L}$ over many iterations the trends are washed out and inconsistent. After further inspection on why the high oscillations exist in the lift coefficient it was discovered that the wing appeared to stall on the upper surface just forward of the slot. This stall was shown to be time dependent and thus associated with the oscillations in $C_{L} \cdot{ }^{45}$

This stall is most likely due to $C_{p}^{*}$ stall which results in gradual loss of suction over the upper surface of the airfoil. This is described in detail first by Abramson and Rogers, then followed up in some computational research by Linton. ${ }^{46,5}$ The stall is reported to be highly regular in its unsteadiness and appears to be inevitable at high $C_{\mu}^{\prime} s{ }^{5}$ This unsteady behavior becomes more exaggerated when observing higher $C_{\mu}$ 's. $C_{p}^{*}$ stall has been observed in both experimental and computational work. Some solutions were run at a range of lower $C_{\mu}$ 's and the stall becomes less prevalent as the $C_{\mu}$ is reduced. This stall can be seen in Figure 5.4.1.

The $C_{p}^{*}$ stall does not affect the deflection angle of the thrust if measured from streamlines released from the engine centerline. This is proved by comparing different solutions throughout a period of an oscillation in $C_{L}$. The change in deflection angle is less than $0.1^{\circ}$. This small change in deflection is due to the relatively small momentum change in the flowfield over the period of the oscillation. The deflection angle is 


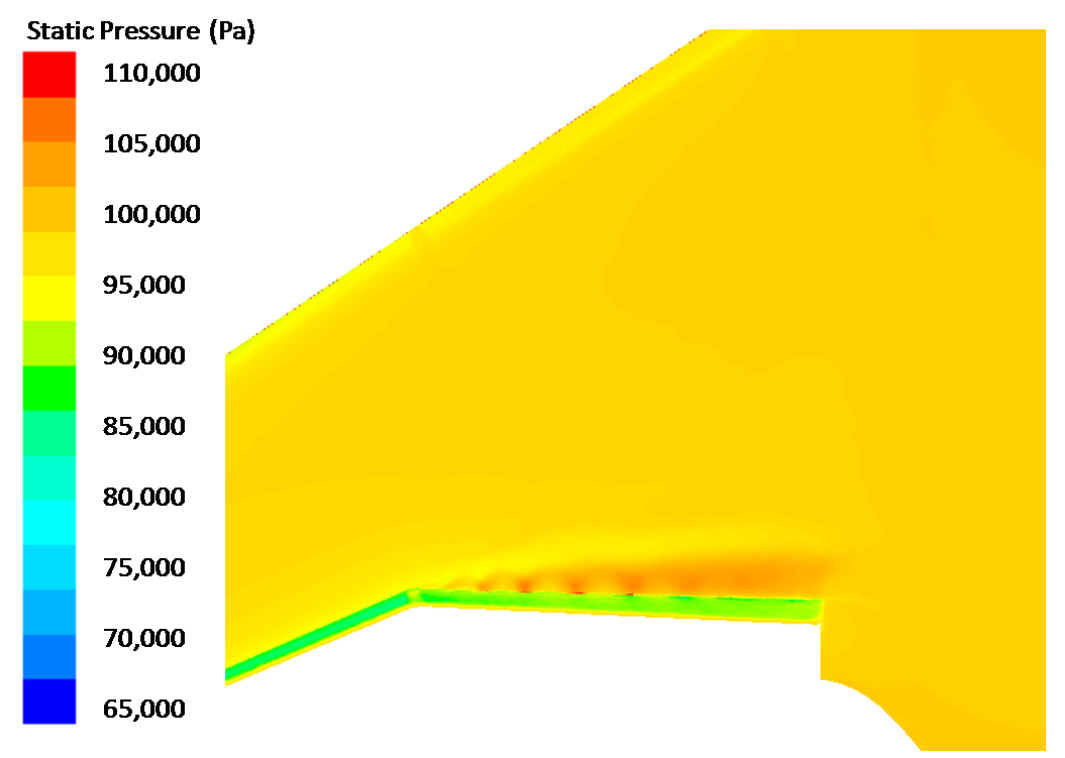

Figure 5.4.1: Static pressure contours showing separation upstream of slot exit.

measured according to the schematic described in Figure 5.4.2.

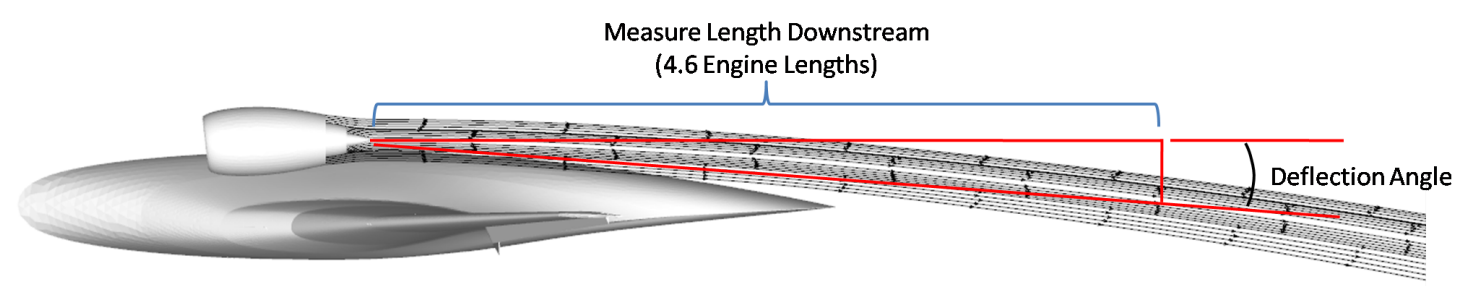

Figure 5.4.2: Schematic showing how to measure engine deflection angle.

\subsection{Solution Details}

Early on it was discovered that engine entrainment is very low when the freestream speed exceeds ground roll. Thus it was advantageous to focus on speeds that would be considered ground roll speeds. This allows for high $C_{\mu}$ 's that are capable of entraining the engine flow. At higher speeds the $C_{\mu}$ 's drop off rapidly because $C_{\mu}$ is non-dimensionalized by dynamic pressure $\left(q_{\infty}\right)$ see Equation 4.3.1. 
As previously discussed the momentum coefficient $C_{\mu}$ for past experiments was theoretically infinite because the tests were run under static conditions. For all of the results presented in this paper the $C_{\mu}$ is held constant at 0.50 . This is just below the maximum theoretical $C_{\mu}$ based on the given slot geometry and the selected freestream conditions. A $C_{\mu}$ of 0.50 gives the best chance of seeing maximum engine entrainment for the given setup. The slot geometry provided difficulty in obtaining the exact conditions at the slot exit. The slot geometry contained aerodynamic and geometric twist, so obtaining the exact surface integral at the slot exit was impossible. The slot conditions are important to calculating the solution $C_{\mu}$ so line integrals are taken of the local conditions at the slot and then the $C_{\mu}$ is calculated using isentropic calculations.

\subsection{Engine Entrainment Results}

The main goal of this paper is to relate engine location and thrust/momentum parameters to the amount of vectored thrust that can be achieved by changing the

engine placement. A secondary goal was to move towards flight conditions that are closer to realistic flight operation.

The results for 10 different engine locations are shown in Figure 5.6.1. The trends show that the further forward and closer to the surface of the engine exit is located the higher the deflection angle of the thrust. This is intuitive and the trends are consistent enough that with a relatively low amount of points one could approximate 
the deflection angle rather easily. The deflection angle is more sensitive to engine height than chord exit location. This is probably due to the Coandã effect being more effective at entraining the engine flow as the engine moves upstream and closer to the surface.

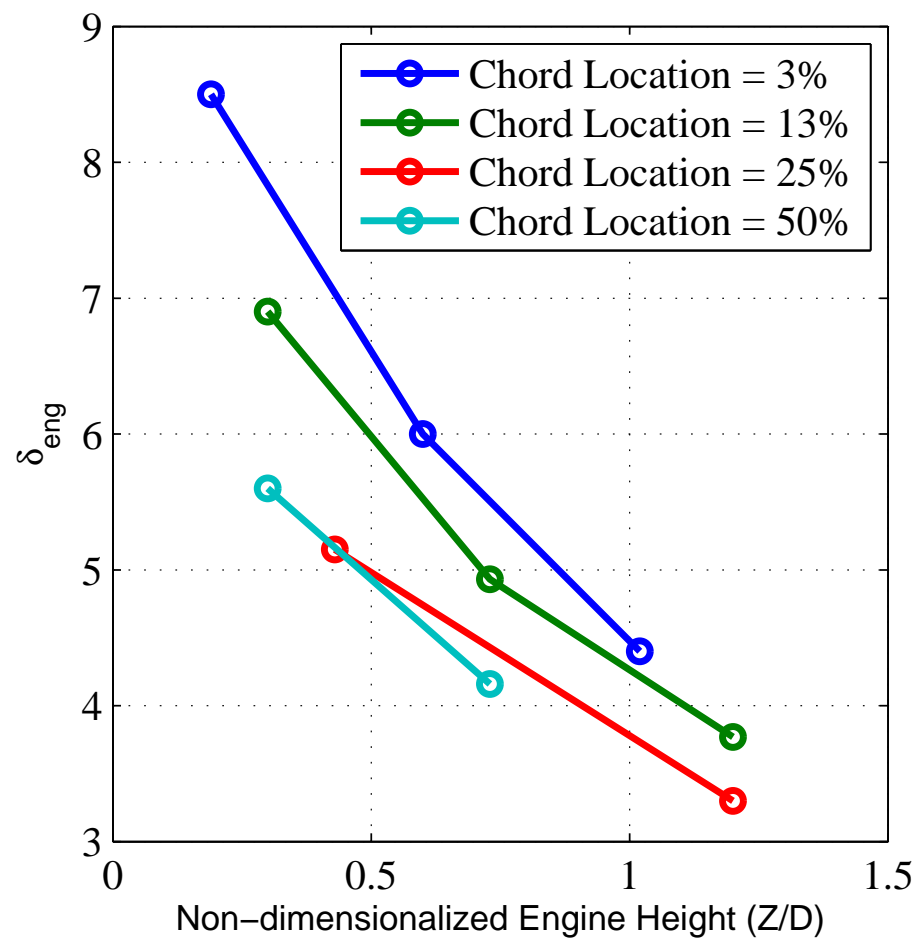

Figure 5.6.1: Graph showing engine deflection based on different engine locations.

The further forward the engine is placed the lower momentum the exhaust contains by the time it reaches the slot. The results for engine height follow what would be expected. The higher the engine is, the lower the deflection angle would be. This is shown in a series of entrainment pictures for a set of engine heights in Figure 5.6.2. 


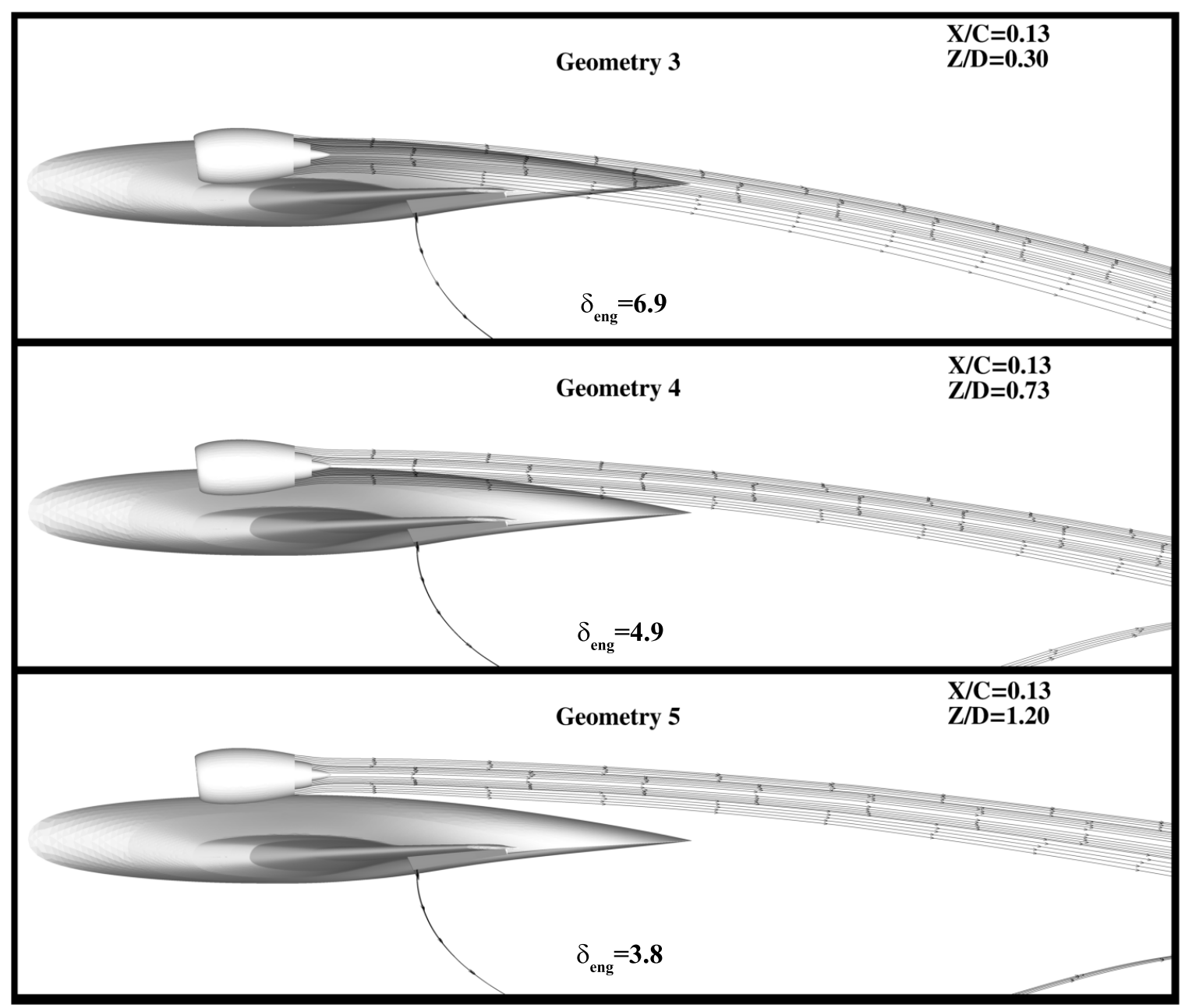

Figure 5.6.2: Streamlines showing engine entrainment for different engine heights.

\section{7 $\quad$ Sensitivities}

Although the trends are clear, it is not evident where the "perfect" location for the engine to be located. While this is a perfect multi-disciplinary problem, some recommendations can be made based on the aerodynamic results presented in this paper. Since, there does not seem to be magic location to place the engine there are some trends that are more sensitive and others and some that can be compromised for other disciplines. 
The most sensitive and perhaps the most important variable measured in this work is the engine height. The engine deflection is very sensitive to engine height. At $3 \%$ chord exit location the engine deflection is reduced by almost half when changing $Z / D$ from 0.2 to 1.0 . This is shown again at $13 \%$ chord when deflection was reduced by almost half when $Z / D$ is taken from 0.30 to 1.20. The trends shown in Figure 5.7.1 appear to be exponentially decreasing with increase in engine height. The trends for both curves were fit using a three point cubic spline.

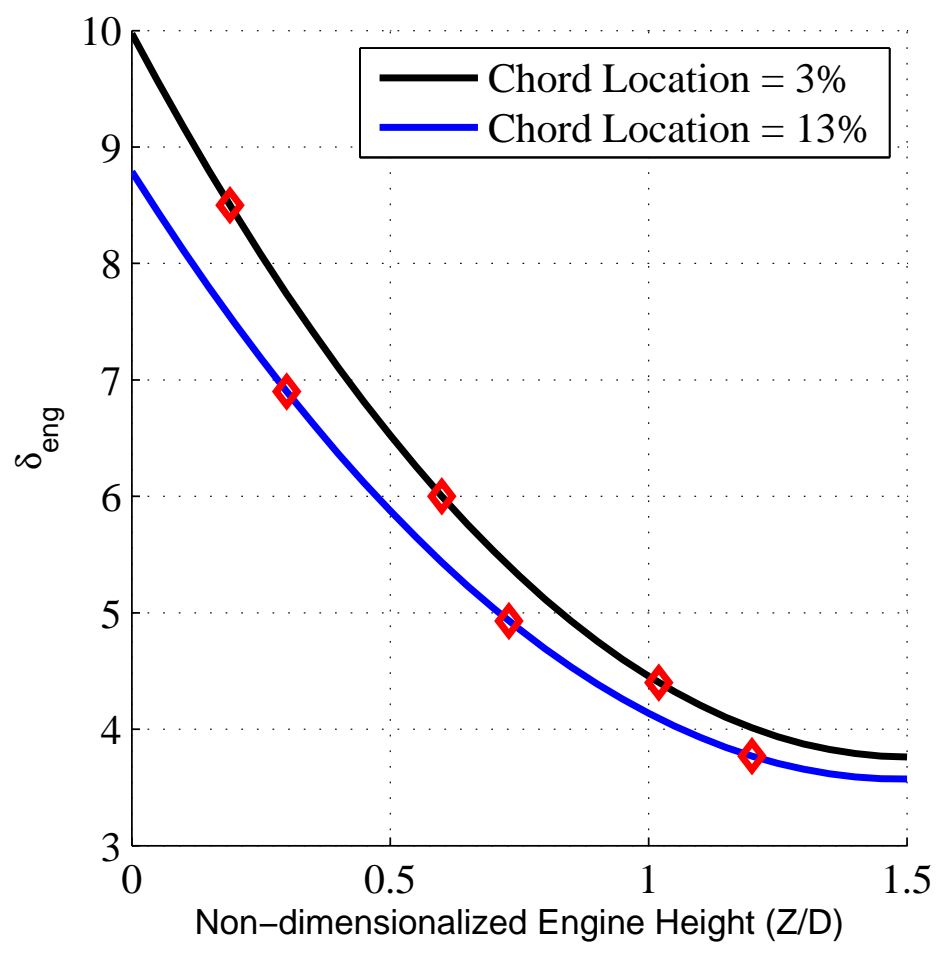

Figure 5.7.1: Engine deflection trends fit using cubic spline.

The Coandã effect seems to be a play a large role in the effectiveness of the deflection. The closer to the wing surface the deflection seems to go up exponentially. This of course, is not a absolute trend because this is only a curve fit but it shows 
some interesting results.

The effect of engine chord exit location on engine deflection angle is much less sensitive than engine height. This being shown in the results by where a change in $37 \%$ chord exit location decreases the deflection by $0.9^{\circ}$. Similarly a decrease of $47 \%$ shows approximately $1.2^{\circ}$ change in deflection angle. This would be a sign that engine exit location could likely be decided by other factors such as noise and structural requirements.

\subsection{Engine Deflection Trends}

The trending $\delta_{\text {eng }}$ curve as $C_{\mu}$ shows an exponential behavior leveling off at a maximum $\delta_{\text {eng }}$ at high $C_{\mu}$ 's. These are the same engine deflection trends that are observed in both $3 \mathrm{D}$ and $2 \mathrm{D}$ validation efforts in Section 4.4. A $C_{\mu}$ sweep of 3 points is taken on Geometry 6 showing this trend. The results are plotted in Figure 5.8.1.

The trend is interesting because it shows that for this configuration the maximum engine deflection for "infinite" $C_{\mu}$ would be approximately $6^{\circ}$. This is low considering the engine is low and forward. This falls well below the high engine deflections shown in the QSRA's static test. The geometry for the QSRA test assists in the engine deflection quite a bit. It would be very intriguing for the QSRA aircraft to be built into a flying test vehicle to evaluate the performance of a viable configuration. This would be a true test of the impact of a coupled USB/CCW configuration.

It is important to point out that very little increase in deflection is shown when 


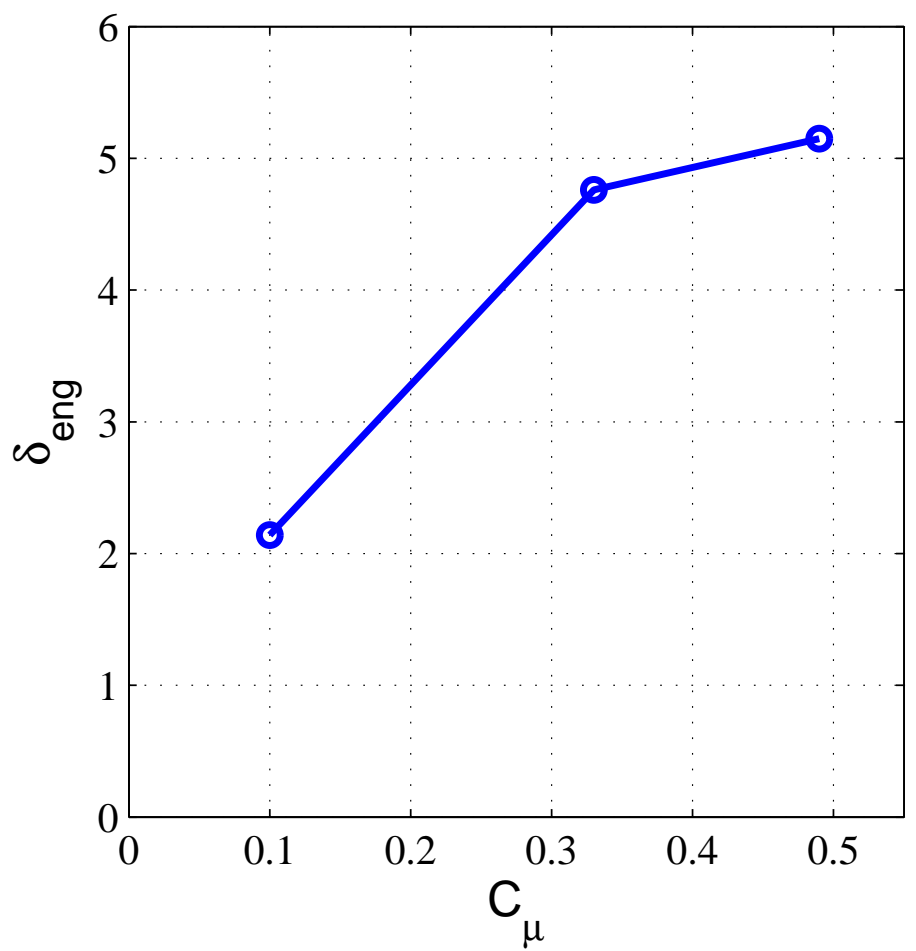

Figure 5.8.1: $C_{\mu}$ sweep for Geometry 6.

increasing the $C_{\mu}$ from 0.33 to 0.50 . The $C_{\mu}$ increase only carries a $0.4^{\circ}$ increase in deflection angle. Deflection angle levels off rapidly at higher $C_{\mu}$. This shows that there is not a large deflection gain from achieving high $C_{\mu}$ values.

\subsection{QSRA Test Comparison}

Section 1.2.3 discusses in detail the Quiet Short-Haul Research Aircraft (QSRA) full scale static ground test of an engine deflection configuration. ${ }^{20}$ The test claims very high engine deflections in its abstract and showed how powerful the system can be. There are a number of reasons that the deflection angles of $40-90^{\circ}$ were not able to be achieved. Many of the reasons were that the QSRA static test were run under 
idealized conditions.

First, the tests was run under static conditions. This means that using the $C_{\mu}$ calculation in Equation 4.3.1 the theoretical $C_{\mu}$ for these tests would be infinite. While it is apparent that a new way to non-dimensionalize $C_{\mu}$ would be needed to evaluate the momentum out of the slot it is clear that the slot would become much more influential on the engine exhaust without the slot having to entrain the freestream energy as well.

One known change is the movement of the engine from being an upper surface blowing engine to one mounted over the wing. The results clearly show that the this movement damaged the effective engine deflection. The QSRA geometry also used a D-nozzle to spread the engine exhaust into a thin sheet across a large span of the wing section. Fences were used to make the flow more two dimensional and focus the exhaust over the span of the circulation control slot.

Even the method with which the engine deflection was measured was misleading. The QSRA forces were measured using load cells attached to the gear. The engine deflection was then calculated from the vectored thrust provided by the CCW and the engine combined. The $\mathrm{CCW}$ alone accounts for $20^{\circ}-25^{\circ}$ of the engine deflection. This lowers the upper engine deflection from the claim of $40^{\circ}-97^{\circ}$ to $15^{\circ}-72^{\circ} .^{21}$ Even those claims are lowered when you see that they were taken at low engine $C_{T}$ 's. In fact, the QSRA data's claim of our adjusted data of $15^{\circ}-72^{\circ}$ of engine deflection was taken from the engine power setting of $25 \%$. Once the results from the QSRA tests 
are reduced to include full engine power, the deflection added due to the coupling of $\mathrm{USB}$ and $\mathrm{CCW}$ are reduced to $10^{\circ}-22^{\circ}$.

Comparing the results presented here against the deflection angles shown on the QSRA static tests, the QSRA achieved $10^{\circ}-22^{\circ}$ of added deflection by coupling a circulation control wing and engine together. This work shows that the deflection range over a number of different locations is $3^{\circ}-8.5^{\circ}$ of deflection. Given all of the idealized conditions in the QSRA tests it is encouraging to obtain the amount of augmented thrust shown in this paper. With optimization of the system, increased deflection may be available to get closer to the deflection achieved by the USB/CCW QSRA combination. While a flight test of this configuration was researched and discussed, it was never flown. ${ }^{47,19}$

Once the added deflection from the QSRA is reduced to a more reasonable value of $10^{\circ}-22^{\circ}$ it compares much better with the results for this test. Given that the QSRA configuration was built to achieve maximum deflection and not having to overcome the added freestream momentum, it is reasonable to expect the QSRA to outperform this geometry by $7^{\circ}-13.5^{\circ}$. 


\section{Chapter 6}

\section{Final Remarks}

\subsection{Conclusions}

This papers shows that there is still some engine deflection with an over the wing engine and the best location for the maximum deflection is forward and low close, to the upper surface of the wing. The deflection is significantly lower than the claims made by some papers in the past which coupled USB engines with circulation control wings. It was discovered that the deflection claims made in the past were made under idealized test conditions and not necessarily real world flight conditions. This paper approached the problem with more realistic flight conditions.

In the introduction it was stated that the coupling of the USB and CCW together increased the deflection of the engine by $10-22^{\circ}$ (adjusted data) depending on the strength of the CCW blowing in the experiment. Keep in mind that this data includes the "infinite" $C_{\mu}$ that cannot be related to real flight conditions. The maximum deflection angle of the OWE/CCW analysis was $8.5^{\circ}$ which is $39 \%$ of the USB/CCW claims. The number would get closer if the static test was to be turned into dynamic 
flight test.

The conclusion being that while the OWE/CCW combination cannot match the performance of the USB/CCW concept, it does show some promising results that could be used to increase takeoff performance while reducing scrub drag in cruise. With that said this is not a magic configuration that is going to significantly reduces take-off and landing distances. The added complexity of this aircraft would need to be studied from many different disciplines to determine if it is a viable aircraft. This study shows only one aspect of a very difficult aircraft design problem.

\subsection{Future Work}

There would be a number of topics that would be interesting to look into if someone wanted to continue this research.

It would be interesting to analyze the effect of the ground plane on engine deflection. See how much wall bounding the aircraft would hinder the performance of the CCW. Engine deflection may be less, but would it be made up for with the added benefit of the wing in ground effect?

Would the "float" airplanes experience on landing, once the plane comes into ground effect, be better or worse? What would be the overall effect of a CCW with controllability?

Another research area would be to perform a deep and thorough investigation into the cause of the separation forward of the trailing edge slot. What are the causes and 
what can be done to keep the wing from having such large lift oscillations?

It would be interesting to have someone study the drag effects based on engine location and see where there may be a compromise between the cruise performance while still maintaining some engine deflection for takeoff and landing. 


\section{Bibliography}

[1] White, F. M., Viscous Fluid Flow, McGraw-Hill, 2006.

[2] Ansys Inc., Fluent 6.3 User's Guide, Sept 2006.

[3] Wilcox, D. C., Turbulence Modeling for CFD, DCW Industries, 1998.

[4] "NASA Government Website," .

[5] Linton, S. W., "Computation of the Poststall Behavior of a Circulation Control Airfoil," Journal of Aircraft, Vol. 31, 1994, pp. 1273-1280.

[6] Englar, R. J., Nichols, Jr, J. H., Harris, M. J., Eppel, J. C., and Shovlin, M. D., "Circulation Control Technology Applied to Propulsive High Lift Systems," Tech. Rep. 841497, SAE Technical Paper Series, Oct. 1984.

[7] SLO, C. P. S. U., Institute, G. T. R., and Engineering, D., "The Integrated Modeling and Verification of a Hybrid Wing-Body Low Noise ESTOL Aircraft," NASA Contract NNL07AA55C, Oct. 2008.

[8] Jones, G. S., Lin, J. C., Allan, B. G., Milholen, W. E., Rumsey, C. L., and Swanson, R. C., "Overview of CFD Validation Experiments for Circulation Con- 
trol Applications at NASA," Tech. Rep. NASA 20080031119, NASA Langley Research Center, 2008.

[9] Storm, T. M. and Marshall, D. D., "Assessing the v2- Turbulence Models for Circulation Control Applications," Tech. rep., AIAA, Dec. 2009.

[10] Marcos, J. and Marshall, D., "Computational and Experimental Comparison of a Powered Lift, Upper Surface Blowing Configuration," Tech. rep., AIAA, 2010.

[11] Chambers, J. R., "Innovation in Flight," Tech. rep., National Aeronautics and Space Administration, 2005.

[12] Lan, C. E. and Campbell, J. F., "Theoretical Aerodynamics of Upper-SurfaceBlowing Jet-Wing Interaction," Tech. rep., National Aeronautics and Space Administration, 1975.

[13] Coe, P. L. J. and Fournier, P. G., "Application of Powered Lift Concepts for Improved Cruise Efficiency of Long-Range Aircraft," Tech. Rep. SP-406, NASA, 1976.

[14] Englar, R. J., Trobaugh, L. A., and Hemmerly, R. A., "STOL Potential of the Circulation Control Wing for High Performance Aircraft," Journal of Aircraft, Vol. 15, No. 77-578, March 1978, pp. 175-181.

[15] Englar, R. J., "Circulation Control for High Lift and Drag Generation on STOL Aircraft," Journal of Aircraft, Vol. Vol. 12, No. No. 5, May 1975, pp. 457-463. 
[16] Englar, R. J. and Williams, R. M., "Design of Circulation Control Stern Plane for Submarine Applications," Tech. rep., Naval Ship Reseach and Development Center, Bethesda, Maryland, March 1971.

[17] Pugliese, A. and Englar, R., "Flight Testing the Circulation Control Wing," AIAA Aircraft Systems and Technology Meeting, Vol. 79, AIAA, AIAA, 1290 Avenue of the Americas, New York, NY 10019, August 1979.

[18] Englar, R. J., "Development of Circulation Control Technology for Powered-Lift STOL Aircraft," Tech. rep., Lockheed Georgia Company, May 1987.

[19] Englar, R., "A Potential Flight Evaluation of an Upper-SurfaceBlowing/Circulation-Control-Wing Concept," NASA Ames Research Center, Vol. N88, 1986, pp. 539-567.

[20] Eppel, J. C., Shovlin, M. D., Jaynes, D. N., Englar, R. J., and Nichols, J. H., "Static Investigation of the Circulation Control Wing/Upper Surface Blowing Concept Applied to the Quiet Short-Haul Research Aircraft," Tech. rep., NASA Technical Memo 84232, July 1982.

[21] Shovin, M. D., Englar, R. J., Eppel, J. C., and Nichols, J. H., "Large-Scale Static Investigation of Circulation-Control-Wing concepts Applied to Upper-SurfaceBlowing Aircraft," Tech. Rep. 2684, NASA, 1987. 
[22] Golden, R. M. and Marshall, D. D., "Design and Performance of Circulation Control Dual Radius Flap System," Tech. rep., AIAA, Dec. 2009.

[23] Englar, R. J. and Huson, G. G., "Development of Advanced Circulation Control Wing High-Lift Airfoils," Tech. rep., David W. Taylor Naval Ship Research and Development Center, Jan. 1983.

[24] Fujino, M. and Kawamura, Y., "Wave-Drag Characteristics of an Over-the-Wing Nacelle Business-Jet Configuration," Journal of Aircraft, Vol. 40, No. 6, Nov.Dec. 2003, pp. 1177-1184.

[25] Connell, S. D., Sober, J. S., and Lamson, S. H., "GridGenerationandSurfaceModelingforCFD," ProceedingsoftheSurfaceModeling,GridGeneration, andRelatedIssuesinComputationalFluidDynamicsWorkshop, NASALewisResearchCenter, NASAConferencePublication, Cleveland, OH, May 1995, p. 29.

[26] Webster, B. E., Shephard, M. S., Rusak, Z., and Flaherty, J. E., "AutomatedAdaptiveTime DiscontinuousFiniteElementMethodforUnsteadyCompressibleAirfoilAerodynamics," AIAA Journal, Vol. 32, No. 4, April 1994, pp. 748.

[27] Yerry, M. A. and Shephard, M. S., "Three-Dimensional Mesh Generation by Modified Octree Technique," International Journal for Numerical Methods in Engineering, Vol. 20, 1984, pp. 1965-1990. 
[28] Shephard, M. S. and Georges, M. K., "Three-Dimensional Mesh Generation by Finite Octree Technique," International Journal for Numerical Methods in Engineering, Vol. 32, 1991, pp. 709-749.

[29] Owen, S., "A Survey of Unstructured Mesh Generation Technology," March 2010.

[30] Lohner, R., Paresh, P., and Clyde, G., "Interactive Generation of Unstructured Grid for Three Dimensional Problems," Numerical Grid Generation in Computational Fluid Mechanics, Vol. 88', 1988, pp. 687-697.

[31] Lohner, R., "Progress in Grid Generation via the Advancing Front Technique," Engineering with Computers, Vol. 12, 1996, pp. 186-210.

[32] Celik, I. B., Ghia, U., Roache, P. J., and Freitas, C. J., "Procedure for Estimation and Reporting of Uncertainty Due to Discretization in CFD Applications," Journal of Fluid Mechanics, 2008.

[33] ANSYS, I., "ANSYS FLUENT," .

[34] Tannehill, J. C., Anderson, D. A., and Pletcher, R. H., Computational Fluid Mechanics and Heat Transfer Second Edition, Taytor and Francis, 1997.

[35] Barths, H., Peters, N., Brehm, N., Mack, A., Pfitzner, M., and Smiljanovski, V., "Simulation of pollutant formation in a gas-turbine combustor using unsteady flamelet," Symposium on Combustion, Vol. 27, 1998, pp. 1841-1847.

[36] Hinze, J. O., Turbulence 2nd Ed, McGraw-Hill, 1975. 
[37] Bardina, J. E., Huang, P. G., and Coakley, T. J., "Turbulence Modeling Validation, Testing, and Development," Tech. rep., NASA Technical Memerandum 110446, 1997.

[38] Menter, F. R., "Two Equation Eddy-Viscosity Turbulence Models for Engineering Applications," AIAA Journal, Vol. 32, 1994, pp. 1598-1605.

[39] Allan, B. G., Jones, G. S., and Lin, J. C., "Reynolds-Averaged Navier-Stokes Simulation of a 2D Circulation Control Wind Tunnel Experiment," Tech. rep., AIAA, 2011.

[40] Storm, T. M., Assessing the v2-f Turbulence Models for Circulation Control Applications, Master's thesis, California Polytechnic State Universit San Luis Obispo, 2010.

[41] Lee-Rausch, E. M., Vasta, V. N., and Rumsey, C. L., "Computational Analysis of Dual Radius Circulation Control Airfoils," Tech. rep., AIAA, 2006.

[42] Englar, R. J., Blaylock, G. H., and Gaeta, R. J., "Recent Experimental Development of Circulation Control Airfoils and Pneumatic Powered Lift," Tech. rep., AIAA, 2010.

[43] Marcos, J. M. and Marshall, D., "Improved Computational and Experimental Validation Using Different Turbulence Models," Tech. rep., AIAA, 2010. 
[44] Baker, W. J. and Paterson, E. G., "Simulation of Steady Circulation Control for a General Aviation Circulation Control (GACC) Wing," Tech. rep., Pennsylvania State University, 2004.

[45] Lidchtwardt, J. A. and Marshall, D. D., "Investgation of the Unstready Behavior of a Circulation Control Wing Using Computational Fluid Dynamics," Tech. rep., AIAA, 2011.

[46] Abramson, J. and Rogers, E. O., "High Speed Characteristics of Circulation Control Airfoils," AIAA Paper 83-0265, Jan. 1983.

[47] Riddle, D. W. and Eppel, J. C., "A Potential Flight Evaluation of and UpperSurface-Blowing/Circulation-Control-Wing Concept," Tech. Rep. N88-17609, NASA-Ames Research Center, 1986. 